\title{
Simulation of Ductile Crack Propagation in
}

\section{Pipeline Steels Using Cohesive Zone Modeling}

\author{
Written by \\ Andrew John Dunbar, B.Eng. \\ a thesis submitted to \\ the Faculty of Graduate Studies and Research in partial \\ fulfillment of the requirements for the Degree of Masters in \\ Applied Science, Mechanical
}

Ottawa-Carleton Institute for

Mechanical and Aerospace Engineering

Department of

Mechanical and Aerospace Engineering

Carleton University

Ottawa, Ontario

August, 2011

(C) Copyright

2011, Andrew Dunbar 


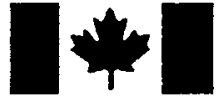

Library and Archives

Canada

Published Heritage

Branch

395 Wellington Street

Ottawa ON K1A ON4

Canada
Bibliothèque et

Archives Canada

Direction du

Patrimoine de l'édition

395 , rue Wellington

Ottawa ON K1A ON4

Canada
Your file Votre référence

ISBN: 978-0-494-83055-0

Our file Notre référence

ISBN: $978-0-494-83055-0$
NOTICE:

The author has granted a nonexclusive license allowing Library and Archives Canada to reproduce, publish, archive, preserve, conserve, communicate to the public by telecommunication or on the Internet, loan, distribute and sell theses worldwide, for commercial or noncommercial purposes, in microform, paper, electronic and/or any other formats.

The author retains copyright ownership and moral rights in this thesis. Neither the thesis nor substantial extracts from it may be printed or otherwise reproduced without the author's permission.
AVIS:

L'auteur a accordé une licence non exclusive permettant à la Bibliothèque et Archives Canada de reproduire, publier, archiver, sauvegarder, conserver, transmettre au public par télécommunication ou par l'Internet, prêter, distribuer et vendre des thèses partout dans le monde, à des fins commerciales ou autres, sur support microforme, papier, électronique et/ou autres formats.

L'auteur conserve la propriété du droit d'auteur et des droits moraux qui protège cette thèse. $\mathrm{Ni}$ la thèse ni des extraits substantiels de celle-ci ne doivent être imprimés ou autrement reproduits sans son autorisation.
In compliance with the Canadian Privacy Act some supporting forms may have been removed from this thesis.

While these forms may be included in the document page count, their removal does not represent any loss of content from the thesis.
Conformément à la loi canadienne sur la protection de la vie privée, quelques formulaires secondaires ont été enlevés de cette thèse.

Bien que ces formulaires aient inclus dans la pagination, il n'y aura aucun contenu manquant. 


\begin{abstract}
Finite element simulations of ductile crack propagation are carried out using cohesive zone modeling. Two sets of materials are modeled. The first material set was defined by Tvergaard and Hutchinson (1992), and has four traction-separation (TS) laws of varying strength and toughness. The second set is modeled after X70 pipeline steel, and is referred to as C2 steel. Three TS laws are used to model this C2 material. The specimens analyzed include small-scale yielding (SSY) and drop-weight tear test (DWTT). The fracture propagation characteristics and CTOA values are obtained. It is shown that cohesive zone models can be successfully used to simulate ductile crack propagation and to numerically measure CTOAs.
\end{abstract}

From SSY simulations, steady-state CTOAs for the TH materials were measured to range from $2^{\circ}-6^{\circ}$. The CTOAs for the $\mathrm{C} 2$ materials were higher than those of the $\mathrm{TH}$ materials due to higher fracture resistance, but no steady-state values were obtained from the SSY simulations. From simulations of the DWTT specimens, steady-state CTOA values for the TH materials ranged from $2^{\circ}-4.5^{\circ}$, and therefore had good agreement with the SSY results. Estimated steadystate CTOA values for the $\mathrm{C} 2$ materials ranged from $14^{\circ}-26^{\circ}$. The measured CTOAs agree reasonably well between the SSY and DWTT simulations for both the TH and C2 material sets, indicating that these values are transferable among specimens. Direct comparisons of the CTOAs obtained from DWTT simulations to the experimental observations $\left(12.4^{\circ}-18.5^{\circ}\right)$ have reasonable agreement as well. 


\section{Acknowledgements}

I would like to thank my thesis supervisor, Professor Xin Wang. This thesis wouldn't have been possible without his invaluable insight and guidance. He never failed to provide encouragement and moral support when necessary, and his teachings during both this degree and my undergrad will not be forgotten.

I am grateful for the financial support from CANMET-MTL, Carleton University and NSERC, that I received to undertake this research project.

I would also like to thank the fast fracture team at CANMET-MTL, as their direction, advice, and wisdom made this thesis possible. Thanks to Bill Tyson, Su Xu, and George Roy. 


\section{Table of Contents}

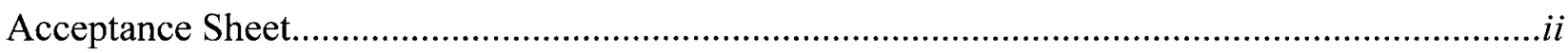

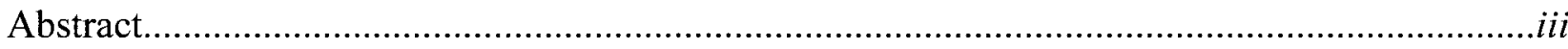

Acknowledgements...........................................................................................................

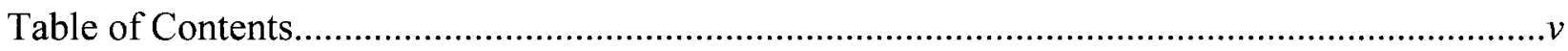

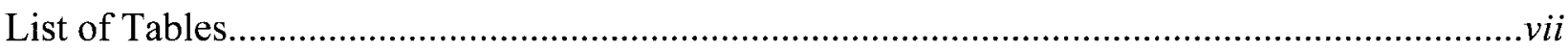

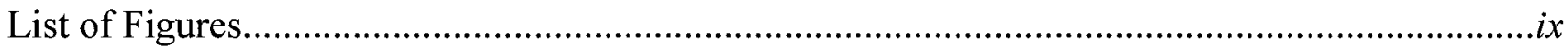

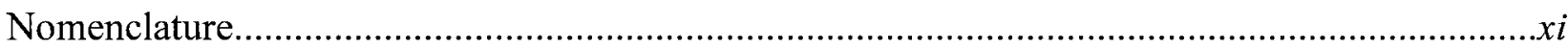

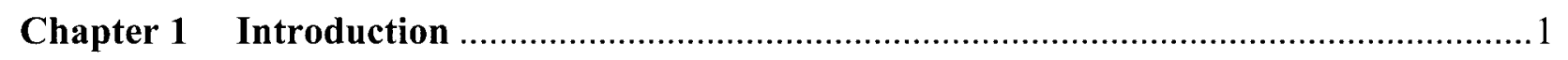

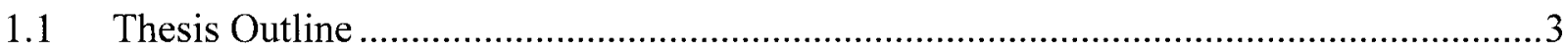

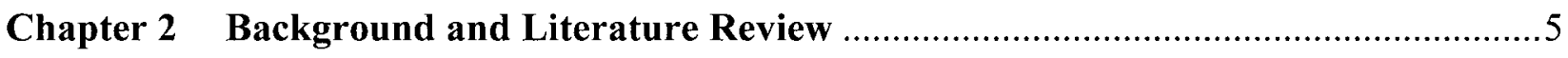

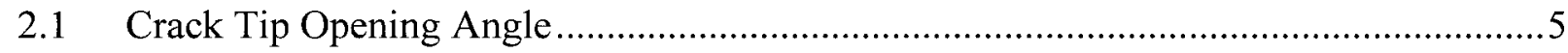

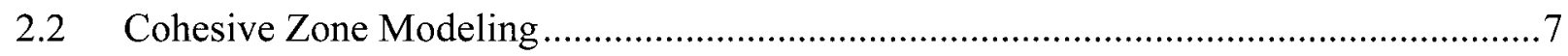

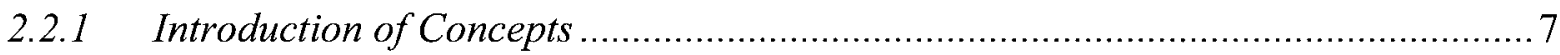

2.2.2 Implementation of Cohesive Zone Modeling ..................................................... 10

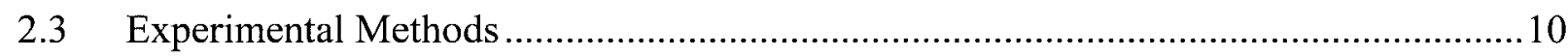

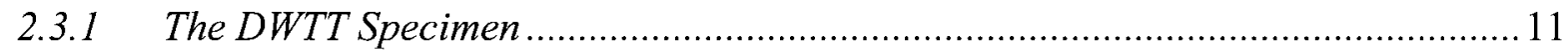

2.3.2 Experimental Setup and Data Collection …………………................................ 11

2.3.3 Measuring the Crack Tip Opening Angle ........................................................... 13

Chapter 3 Finite Element Implementation of Cohesive Zone Modeling ............................24

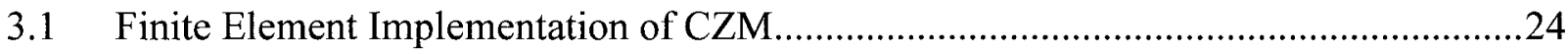

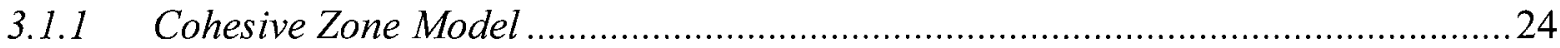

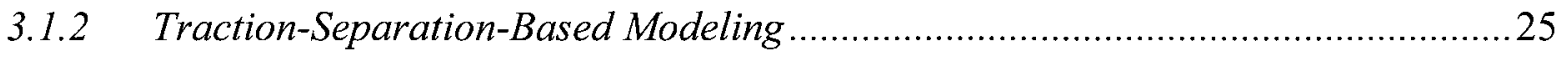

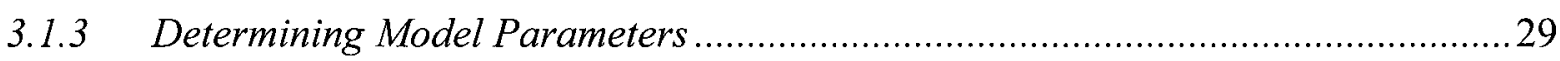




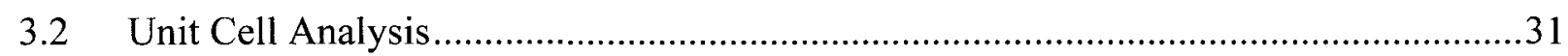

Chapter 4 FE Simulation of Small-Scale Yielding Models …….......................................47

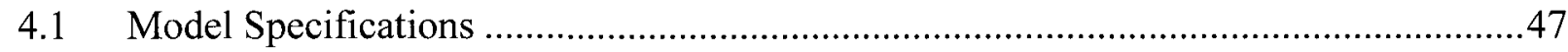

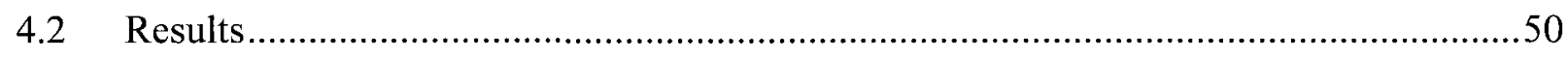

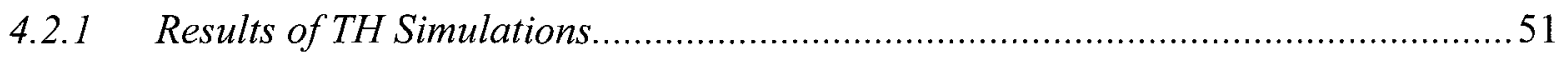

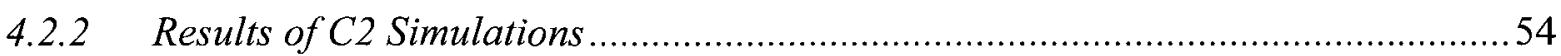

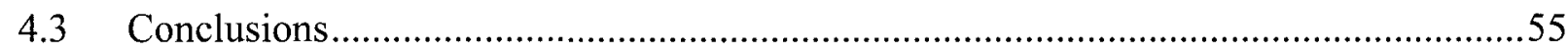

Chapter 5 FE Simulation of Drop-Weight Tear Tests ...................................................

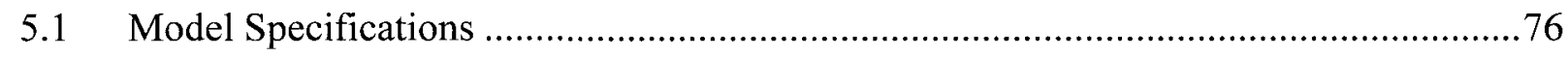

5.2 Simulation Using Plane Strain Elastic-Plastic Model.....................................................79

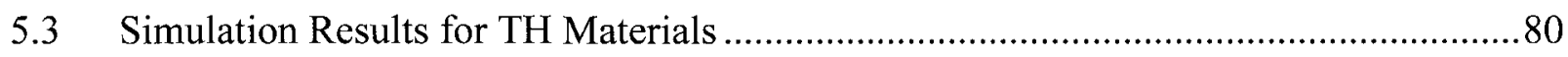

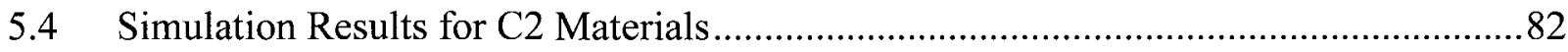

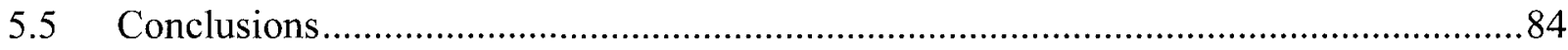

Chapter 6 Conclusions and Recommendations …….......................................................105

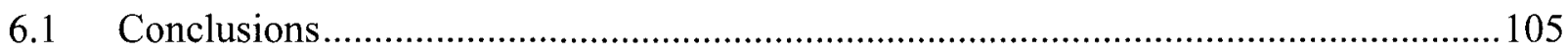

6.2 Recommendations for Future Work ......................................................................... 107

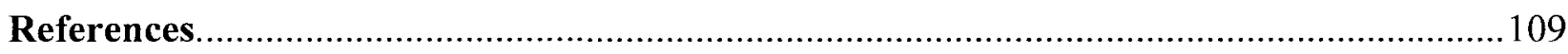




\section{List of Tables}

Chapter 2

Table 2.1: P vs. LLD Data - C2 Steel - Corrected for Compliance (Xu, 2010a). 15

\section{Chapter 3}

Table 3.1: TH Material Plastic Strain..... .35

Table 3.2: C2 steel's Chemical Composition ......................................................................36

Table 3.3: C2 steel's Average Transverse Tensile and Charpy Properties (3 samples) ...............36

Table 3.4: C2 steel's Plastic Strain vs. Stress - as Input in Abaqus.................................... 37

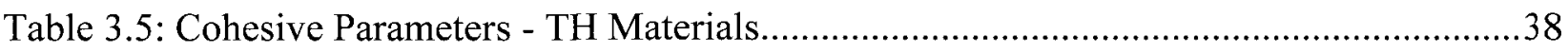

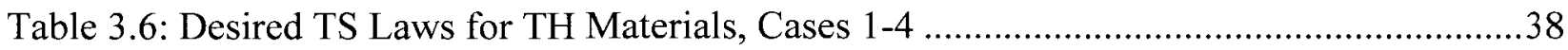

Table 3.7: Measured Results from Unit Cell Analyses for TH Materials .................................39

Table 3.8: Cohesive Parameters - C2 Materials ....................................................................40

Table 3.9: Desired TS Laws for C2 Materials, Cases 1-3 ....................................................40

Table 3.10: Measured Results from Unit Cell Analyses for C2 Materials................................41

\section{Chapter 4}

Table 4.1: Displacement Field - Maximum Applied Displacements.......................................56

Table 4.2: J-Integral Data for TH Materials ............................................................5

Table 4.3: Model Parameters - (a) TH Materials, (b) C2 Materials .......................................58

Table 4.4: SSY Sample Resistance Curve Data for TH1 Material..........................................59

Table 4.5: SSY Sample Resistance Curve Data for TH2 Material.......................................60

Table 4.6: SSY Sample Resistance Curve Data for TH3 Material.......................................61

Table 4.7: SSY Sample Resistance Curve Data for TH4 Material..........................................62

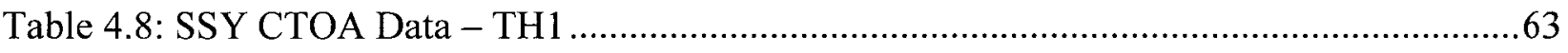

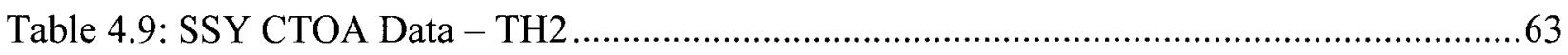

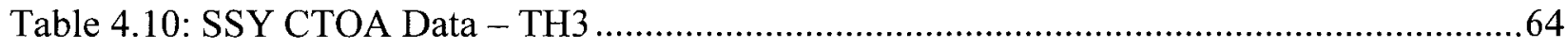

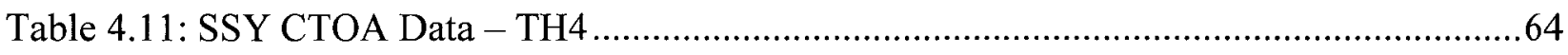

Table 4.12: SSY Sample Resistance Curve Data for C2_1 Material ......................................65 
Table 4.13: SSY Sample Resistance Curve Data for C2_2 Material .......................................66

Table 4.14: SSY Sample Resistance Curve Data for C2_3 Material ......................................67

Table 4.15: SSY CTOA Data - C2 Materials ....................................................................68

Chapter 5

Table 5.1: P vs. LLD Data from CANMET - C2 Steel - Corrected for Compliance..................86

Table 5.2: P vs. LLD Sample data: Plane Strain Simulation..................................................87

Table 5.3 (a) - (d): Sample P vs. LLD data - TH Materials ...............................................8 88

Table 5.4 (a) - (d): Sample $\Delta \mathrm{a}$ vs. LLD data - TH Materials ..............................................8

Table 5.5 (a) - (d): Sample CTOA vs. $\Delta \mathrm{a}$ data - TH Materials ..........................................90

Table 5.6 (a) - (c): P vs. LLD data - C2 Materials ...........................................................91

Table 5.7 (a) - (c): $\Delta$ a vs. LLD data - C2 Materials ................................................. 92

Table 5.8 (a) - (c): CTOA vs. $\Delta$ a data - C2 Materials.......................................................93 


\section{List of Figures}

\section{Chapter 2}

Figure 2.1: Partially Fractured Charpy Sample (post test) (Xu et al, 2004) ....................................16

Figure 2.2: Representation of the Ductile Failure Process by CZM (Cornec, 2003) .....................16

Figure 2.3: Four Examples of Various Shapes of TS Laws (Alfano, 2006)..................................17

Figure 2.4: C(T) Test: P vs. LLD Curves for Various TS Law Shapes (Alfano, 2006).................17

Figure 2.5: Trapezoidal Traction-Separation Law (Tvergaard and Huchinson, 1992) .................18

Figure 2.6: DWTT Geometry (a) Full Specimen, (b) Notch Geometry .......................................19

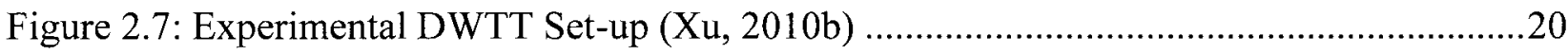

Figure 2.8: P vs. LLD Data for C2 steel - Corrected for Compliance (Xu, 2010a) ......................21

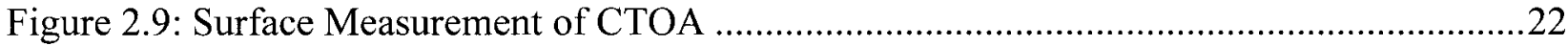

Figure 2.10: Slow-rate-tested X70 Pipe Sample Showing Wavy Crack Flanks ...........................23

\section{Chapter 3}

Figure 3.1 : COH2D4 Element Numbering Conventions (Abaqus, 2008) ................................42

Figure 3.2: Typical Traction-Separation Based Cohesive Zone Model (Abaqus, 2008) ..............42

Figure 3.3: Typical Traction-Separation Response (Abaqus, 2008) .............................................43

Figure 3.4: TH Steel - True Stress-Strain Curve .....................................................................43

Figure 3.5: C2 Steel - True Stress-Strain Curve (Su, 2010a)......................................................44

Figure 3.6: Unit Cell Geometry and Boundary Conditions..........................................................44

Figure 3.7: Unit Cell Analysis, Verification of TS Laws for TH Materials..................................45

Figure 3.8: Unit Cell Analysis, Verification of TS Laws for C2 Materials ..................................46

\section{Chapter 4}

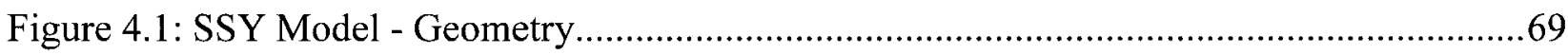

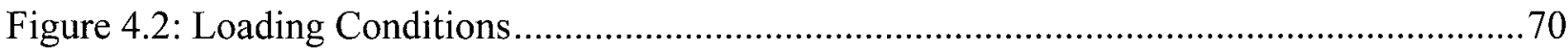

Figure 4.3: Mesh Design - Full Model................................................................................70

Figure 4.4 (a) - (c): Crack Tip Mesh Design …………………................................................. 71

Figure 4.5 : SSY- Normalized Resistance Curves - TH Materials - With Literature Data ...........72 


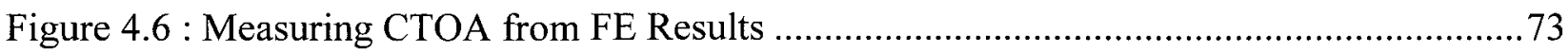

Figure 4.7 : CTOA Data - SSY Model - TH Materials ……………………..............................

Figure 4.8 : SSY- Normalized Resistance Curves - C2 Materials .............................................74

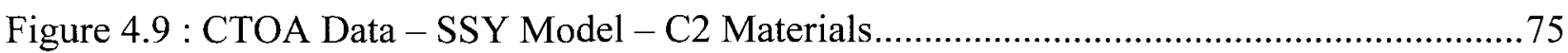

\section{Chapter 5}

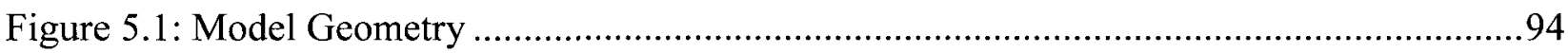

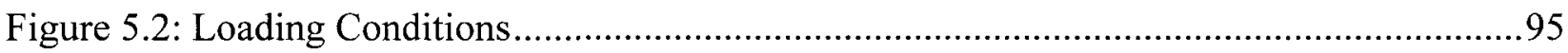

Figure 5.3: Mesh Design - Full Model...............................................................................95

Figure 5.4: Mesh Design - Refinement at Tup .......................................................................96

Figure 5.5: Mesh Design - Refinement at Anvil................................................................... 96

Figure 5.6: Mesh Design - Refinement at Crack Tip............................................................... 97

Figure 5.7: Model Verification - P vs. LLD curves -Plane Strain and Experimental Data..........98

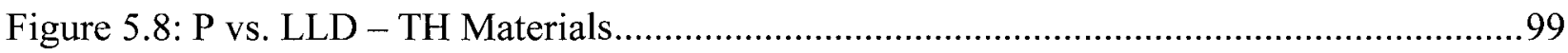

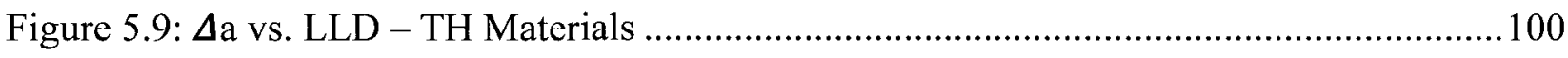

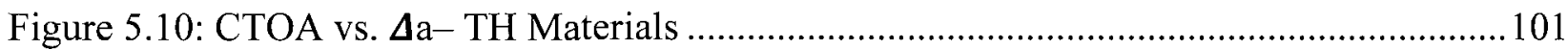

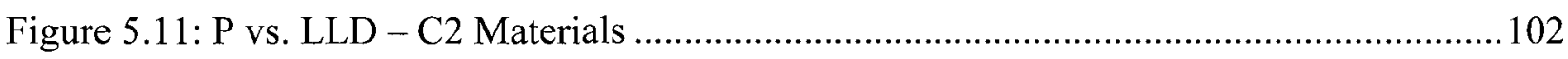

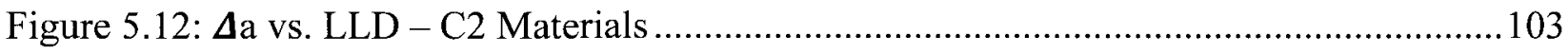

Figure 5.13: CTOA vs. $\Delta \mathrm{a}-\mathrm{C} 2$ Materials ................................................................................. 104 


\section{Nomenclature}

a: crack length

$a_{0}$ : initial crack length

$\mathrm{A}_{\mathrm{o}}$ : radius of the small-scale yielding model

$\mathrm{b}$ : length of the remaining ligament

B: thickness of specimen

Bo: twice the length of cohesive zone

CTOA: crack-tip opening angle

$\mathrm{CTOA}_{\mathrm{c}}$ : critical crack-tip opening angle

$\mathrm{CTOA}_{\mathrm{ss}}$ : crack-tip opening angle during steady-state crack propagation

CTOD: crack-tip opening displacement

CVN: Charpy V-notch energy

CZM: Cohesive Zone Model

D: damage function

DWTT: drop-weight tear test

E: Young's Modulus

$\mathrm{F}$ : force

$\mathrm{G}, \mu$ : Shear Modulus

(5: Griffith criterion for crack advance

$\mathrm{K}_{l l}$ : stiffness of cohesive elements $(\mathrm{i}=\mathrm{n}, \mathrm{s}, \mathrm{t})$

$\mathrm{K}_{\mathrm{r}}$ : applied stress intensity factor

$\mathrm{K}_{\mathrm{o}}$ : critical stress intensity factor

LLD: load-line displacement

$\mathrm{n}$ : strain-hardening exponent of power-law stress-strain representation (defined by E1290-2)

$\mathrm{N}$ : strain-hardening exponent of Ramburg-Osgood stress-strain representation $(\mathrm{N}=1 / \mathrm{n})$

P: load

$\mathrm{R}_{\mathrm{o}}$ : estimated plastic zone size

$\mathrm{r}_{\mathrm{p}}$ : plastic rotation factor

S: span between two load points

SE(B): single-edge bend specimen

S-SSM: simplified single-specimen method for measuring CTOA 
$\mathrm{T}, \mathrm{T}(\delta)$ : traction, traction as a function of separation

$\mathrm{T}_{0}$ or $\hat{\sigma}$ : maximum or peak traction

$u_{x}, u_{y}$ : Displacement in the $\mathrm{x}$ and $\mathrm{y}$ directions

W: width of specimen

$\delta$ : separation

$\delta_{\mathrm{c}}:$ critical separation, $(\mathrm{D}=1.0, \mathrm{~T}=0)$

$\delta_{0}$ : separation that induces damage

$\delta_{m}^{\max }$ : maximum effective separation attained during the complete loading history of the element

$\Delta_{0}$ : the length of the smallest elastic-plastic elements adjacent to the cohesive layer

$\varepsilon$ : strain

$\sigma_{y}$ : yield stress, $0.2 \%$ proof strength at the temperature of the fracture test

$v$ : Poisson's ratio

$\Gamma_{o}$ : fracture energy/ work of separation - (Expressed in MPa $\cdot \mathrm{mm}$, equivalent to $\mathrm{kJ} / \mathrm{m}^{2}$ )

$\Gamma_{\mathrm{r}}$ : crack growth resistance 


\section{Chapter 1}

\section{Introduction}

The increased demand for natural resources such as oil and gas has led researchers to seek higher efficiencies from transmission pipelines. This has led to the development of newer grades of pipeline steel, such as $\mathrm{X} 70, \mathrm{X} 80$, and even $\mathrm{X} 100$, that have higher strength and toughness than previous steels (Horsley, 2003). Unfortunately though, these developments in material technology have caused traditional toughness characterization techniques to lead to nonconservative predictions for growth of axial pipeline cracks (Shim et al, 2010 a,b). New techniques, able to accurately predict fracture in pipelines made from these new grades of steel, are required to confidently design fracture control plans.

The crack tip opening angle (CTOA) has gained wide acceptance as a fracture resistance parameter and standards for its use in fracture prediction have been published by both ASTM (2006) and ISO (2007). The CTOA is a material property that characterizes the toughness of a material. The premise is that stable crack extension occurs at a constant CTOA. The tougher the material is, the higher the CTOA needs to be for a crack to propagate. The CTOA needed to initiate fracture is called the critical crack tip opening angle, $\mathrm{CTOA}_{\mathrm{C}}$. Typically this initiation value is quite high, but it drops down quickly to a constant or steady-state angle, $\mathrm{CTOA}_{\mathrm{SS}}$, after a small amount of crack propagation (Newman et al, 2003).

Measuring the CTOA by performing full-scale pipeline burst tests is too expensive to be practical. Therefore, it is desirable to develop prediction techniques based on data observed from small-scale lab tests using specimens such as the modified double cantilever beam, MDCB, compact tension, $\mathrm{C}(\mathrm{T})$, and single-edge bend, $\mathrm{SE}(\mathrm{B})$. The CTOA measured from these specimens will be transferable to full pipeline geometry (Horsley, 2003). To further develop the applications of CTOA theory to pipeline fracture control plans, it is necessary to build up a database of CTOA information, measured from a variety of specimens, for a variety of high toughness steels, using a variety of measurement techniques. 
There are many techniques for measuring the CTOA, both experimentally and numerically. In this thesis, finite element analyses were performed to numerically measure the CTOA for two different high strength, high toughness, steels. The first steel is referred to as a TH steel, as it is an ideal steel with properties defined by Tvergaard and Hutchinson (1992). The second steel is a real material, referred to as a C2 steel. This pipeline steel was characterized by Xu (2010a). An experimental drop-weight tear test was performed using this C2 steel. Load vs. load-line displacement and CTOA data, measured using optical techniques and the simplified singlespecimen method (Xu et al, 2007), were provided for comparison to the numerical results of the DWTT simulations conducted in this thesis.

The main focus of this thesis is to explore the determination of CTOA using cohesive zone (CZ) models. A CZ model uses a thin layer of cohesive elements along the crack path. These cohesive elements are typically governed by a traction vs. separation (TS) law that defines the element's load transferring capability based on the separation, or strain, of the element. Each element has a peak stress, or traction, that decreases to zero as the element reaches a critical separation. When a cohesive element's load transferring capability is reduced to zero, it is said to have failed. This allows the crack to propagate. Various shapes of TS laws have been proposed but bilinear TS laws were chosen for this thesis. Abaqus/Explicit (2008) is used to perform the cohesive zone modeling in this thesis.

The first step of this thesis was to verify the chosen traction-separation laws through analysis of a unit cell in Abaqus. A total of seven bilinear TS laws were considered, with four used to model the TH material, and three used to model the C2 material. The four TH cases were modeled after those defined by Tvergaard and Hutchinson. Each TS law had different peak tractions and fracture toughnesses.

Following this verification, a small-scale yielding model was used to measure how changes to the peak traction and fracture energy changed the macroscopic response of a model when the 
elastic-plastic material parameters were held constant. This was done by modeling with four different TS laws for the TH material, and three different TS laws for the C2 material. Resistance curves were generated for all seven cases, and the TH cases were compared to the data published by Tvergaard and Hutchinson. CTOA data was also measured for all seven cases.

Finally, drop-weight tear tests were simulated using the $\mathrm{TH}$ and $\mathrm{C} 2$ material sets. The fracture resistance and crack propagation was observed by generating load vs. load-line displacement and crack length vs. load-line displacement curves. The load-line displacement curves generated from the three $\mathrm{C} 2$ simulations were compared with the experimental observations provided by Xu (2010a). CTOA data from the DWTT simulations were compared to CTOA data from the SSY simulations. CTOA data from the C2 DWTT simulations were compared to the experimental observations of Xu et al (2010b).

\subsection{Thesis Outline}

The objective of this thesis is to conduct finite element simulations of ductile crack propagation for a wide range of high strength pipeline steels (four TH steels, three $\mathrm{C} 2$ steels). The specimens analyzed include SSY and DWTT specimens. The fracture resistance curves and CTOA values are obtained. It is shown that cohesive zone models can be successfully used to simulate ductile crack propagation and to numerically measure CTOAs. The CTOAs are found to be transferable between the SSY and DWTT specimens. The CTOAs are comparable to experimentally obtained values.

Chapter 2 provides a review of the theory behind the crack tip opening angle and the cohesive zone model, as well as the experimental techniques. The theory of cohesive zone elements, as well as verification of Abaqus cohesive elements is presented in Chapter 3. Chapter 4 details the results of small-scale yielding simulations performed to test the finite element techniques. Chapter 5 documents the results of DWTT simulations of the TH and C2 materials, and provides 
comparisons of CTOA data between materials and specimens, as well as direct comparison of the CTOA measured from the model to experimental observations for the $\mathrm{C} 2$ steel. 


\section{Chapter 2}

\section{Background and Literature Review}

This chapter will introduce the crack tip opening angle (CTOA), a fracture parameter that has been widely accepted to provide superior prediction capabilities than traditional techniques. Following this, cohesive zone modeling (CZM) will be described as a technique for simulating crack propagation and numerically predicting the CTOA. Finally, the methods used to gather the experimental data that will be used to verify the finite element results will be documented.

\subsection{Crack Tip Opening Angle}

The development of newer grades of pipeline steel, that have higher strength and toughness, was required to increase the efficiency of pipelines, and improve their reliability. These developments have reduced the accuracy of traditional elastic-plastic fracture mechanics techniques and in many cases have led to non-conservative predictions for growth of axial pipeline cracks (Shim et al, 2010 a,b; Horsley, 2003).

The Charpy test has traditionally been used to characterize the toughness of a material. The Charpy V-notch energy (CVN) is then typically used in semi-empirical models to predict minimum arrest toughness to prevent unstable fracture. The Charpy specimen though has some key deficiencies that degrade its prediction abilities for high toughness steels (Shim, 2010a). First, the blunt notch causes resistance data to include energy from crack initiation as well as crack propagation. Secondly, the specimen is usually smaller than the full thickness of a pipeline and toughness has been found to relate to thickness. Additionally, the length of the fracture ligament is not long enough to reach steady-state fracture and fracture resistance has been shown to vary with crack length. Finally, for many of the higher toughness steels, Charpy samples are only incompletely fractured, as in Figure 2.1 (Xu et al, 2004). It is for these reasons that the drop-weight tear test (DWTT) has been proposed as an alternative method to characterize 
fracture resistance for new grades of ductile steels. Experimental data from this technique will be used to validate the numerical modeling results in Chapter 5.

A traditional method for predicting full-scale pipeline fracture speed and minimum arrest toughness is called the Battelle Two-Curve Method (TCM) (Maxey et al, 1976). The TCM empirically combines the influence of factors including the material fracture toughness, gas decompression behaviour, and backfill conditions. Traditionally this method relies on fracture resistance R, a constant material property. Researchers at TransCanada PipeLines Limited and Engineering Mechanics Corporation of Columbus (Shim et al, 2010, a,b) have concluded though that this method is inadequate and have made attempts to modify and improve the method by instead using a speed dependent fracture resistance in order to apply it to newer, tougher, grades of pipeline steel. This is still an empirical method and relies on the ability to accurately produce resistance curves that correspond to the real world application.

The crack tip opening angle, proposed by Rice and Sorensen (1978), has been proposed as an alternative to these previous techniques to characterize fracture resistance. The CTOA has been approved by the International Standards Organization to quantify the resistance to stable crack extension in ISO 22889 (2007). The CTOA is also approved by ASTM International as a method for determining fracture resistance in ASTM E2472 (2006). It is believed that the CTOA measured during stable or steady-state crack propagation is a material property. Therefore, the steady-state CTOA measured during a lab test, a drop-weight tear test (DWTT) for example, should be transferrable to a simulation of crack propagation in a full-scale pipeline.

There are several techniques commonly used for measuring the CTOA, both experimentally and numerically. These include, but are not limited to, optical microscopy, digital image correlation, microtopography, and calculation from finite element results (ISO, 2007). In the current work, CTOAs will be calculated from finite element results of small-scale yielding and drop-weight tear test simulations using a method outlined in Section 4.2.2. For the DWTT simulations in 
Chapter 5, the CTOAs calculated from finite element results will be compared to reported CTOAs that were determined using digital image correlation, as well as a method called the simplified single-specimen method developed by Xu et al (2007). These measurement techniques will be outlined in Section 2.3.3. The steady-state CTOAs determined from the two different models (SSY and DWTT) will be compared, and the feasibility of using cohesive zone modeling to predict reliable steady-state CTOAs will be discussed in Chapter 5.

\subsection{Cohesive Zone Modeling}

\subsubsection{Introduction of Concepts}

The idea of a cohesive layer at a crack tip was originally introduced by Dugdale (1960) and Barenblatt (1962). Since its introduction, the field on cohesive zone modeling has since grown in popularity and is now being used in civil, aerospace, and mechanical engineering applications. Recently there have been many groups researching the use of CZM to predict crack growth in thin sheets for applications such as aircraft fuselage and oil and natural gas transmission pipelines including Keller et al (1999), Chen et al (2003), Chandra et al (2002), Elices et al (2002), Cornec et al (2003), de Borst (2003), and Scheider and Brocks (2006).

The CZM was originally proposed as a phenomenological approach to model brittle fracture by assigning a degradation vs. separation law to a process zone along the crack front. In the real world, ductile fracture occurs through void initiation, growth, and coalescence. By assigning a cohesive or Traction-Separation (TS) law to a layer of cohesive elements this ductile fracture process can be modeled by performing a finite element analysis. A diagram representing this process can be found in Figure 2.2 (Cornec, 2003). Here, T $(\delta)$ represents the traction, T, vs. separation, $\delta, \Delta$ a represents the crack growth, and the subscripts $\mathrm{N}, \mathrm{S}$, and 0 respectively represent normal, shear, and critical values of traction and separation.

A TS law is a progressive damage model that defines the maximum traction based on the separation or strain history of the element. The shape of the TS law can vary depending on the 
application and researchers' methodology. Four examples of TS laws can be found in Figure 2.3 (Alfano, 2006). These include bilinear, linear-parabolic, exponential, and trapezoidal laws.

Regardless of the shape, TS laws all have some important features in common:

- An initial stiffness curve for $\delta<\delta_{0}$,

- A portion of increasing damage and reduced ability to transfer loading (reduced traction) beginning at initiation point $\delta_{0}$; and

- A final separation value, $\delta_{\mathrm{c}}$, after which ability to transfer loading has been completely removed $(T=0)$.

The three defining parameters are the maximum cohesive strength, $\sigma_{0}$ or $T_{0}$, the final separation, $\delta_{\mathrm{c}}$, and the work of separation, $\Gamma_{\mathrm{o}}$, which is represented by the area under the curve. While initially some authors, including Tvergaard and Hutchinson (1992), found minimal influence of the shape, more extensive studies carried out by Chandra et al (2002), Cornec et al (2003) and Alfano (2006) have determined that the shape of the TS law plays an important role in determining the macroscopic results of the fracture process. Figure 2.4 (Alfano, 2006) shows how the four TS shapes defined in Figure 2.3 affect the load vs. displacement curves of a C(T) specimen. While all four TS laws have the same peak traction and work of separation, the peak load predicted in the $C(T)$ specimens varies between laws. This work highlights the importance of choosing a consistent TS law, and modeling with it in various geometries to fully understand its properties. In the present work, only the bilinear shape will be used.

\section{TS Laws as They Relate to EPFM}

The first authors to apply the CZM to ductile fracture were Tvergaard and Hutchinson (1992). They demonstrated the ability of cohesive zone models (CZMs) to predict resistance curves for plane strain, mode I crack growth in small-scale yielding (SSY). This work was an important first step towards developing CZMs that can be used for predictive purposes in design using ductile materials. The TS law proposed by Tvergaard and Hutchinson was a trapezoidal law, as seen in Figure 2.5 (Tvergaard and Hutchinson, 1992). 
It is possible to relate the traction-separation laws to classical fracture mechanics. The Griffith criterion for crack advance, $\mathfrak{F}$, is equal to the crack growth resistance, $\Gamma_{R}(\Delta a)$ :

$$
\mathfrak{G}=\Gamma_{r}(\Delta a)
$$

under conditions of small-scale yielding, where the growth resistance varies with the crack extension due to the changing plastic zone. This can be related to the stress intensity factor, $\mathrm{K}$, at the crack tip by:

$$
\mathfrak{5}=\frac{\left(1-v^{2}\right)}{\mathrm{E}} \mathrm{K}^{2}
$$

where $v$ is Poisson's ratio, $\mathrm{E}$ is Young's modulus, and $\mathrm{K}=\mathrm{K}_{\mathrm{r}}(\Delta \mathrm{a})$, the stress intensity at the crack tip as a function of crack length. The stress-intensity factor at the crack tip can now be directly related to the crack growth resistance by:

$$
\mathrm{K}_{\mathrm{r}}=\sqrt{\frac{\mathrm{E} \Gamma_{r}}{\left(1-v^{2}\right)}}
$$

Therefore, the critical stress intensity factor, $\mathrm{K}_{0}$, that will cause the crack to initiate for a particular TS law can be found by:

$$
\mathrm{K}_{0}=\sqrt{\frac{\mathrm{E} \Gamma_{0}}{\left(1-v^{2}\right)}}
$$

For a trapezoidal separation law the work of separation per unit area, $\Gamma_{0}$, can be written as: 


$$
\Gamma_{0}=1 / 2 \hat{\sigma}\left[\delta_{c}+\delta_{2}-\delta_{1}\right]
$$

where $\hat{\sigma}$ is the maximum traction, $\delta_{c}$ is the critical separation, and $\delta_{1}, \delta_{2}$ are defined in Figure 2.5. For a bilinear separation law, such as the one given in Figure 2.3, it can be written as :

$$
\Gamma_{0}=1 / 2 \hat{\sigma} \delta_{c}
$$

Therefore, the critical stress intensity factor for a CZ model with a bilinear TS law is:

$$
\mathrm{K}_{0}=\sqrt{\frac{\mathrm{E} \cdot \hat{\sigma} \cdot \delta_{c}}{2 \cdot\left(1-v^{2}\right)}}
$$

\subsubsection{Implementation of Cohesive Zone Modeling}

For the present work, implementation of cohesive zone modeling is performed in Abaqus/Explicit (Abaqus, 2008). Section 3.1.2 outlines how Abaqus defines cohesive elements when performing traction-separation-based modeling. Section 3.1.3 outlines the selection of the necessary cohesive parameters to implement specific TS laws in Abaqus. Chapter 4 outlines the process of defining and meshing a cohesive zone model in Abaqus, and Section 4.2.2 outlines the process of measuring the CTOA from finite element results.

\subsection{Experimental Methods}

The crack tip opening angle (CTOA), measured using the drop-weight tear test (DWTT) has been proposed as a fracture parameter that can be used to characterize material toughness. Therefore, the experimental results from DWTTs performed on a C2 (X70) steel will be used to 
verify and calibrate finite element results. Here, the DWTT model is introduced and experimental procedures for obtaining the crack tip opening angle and other experimental data are outlined.

\subsubsection{The DWTT Specimen}

The drop-weight tear test specimen is special case of a single-edge bend specimen (SE(B)). General geometry for the DWTT specimen is found in Figure 2.6 (a) (Xu et al, 2011a). Two anvils are used to support the specimen while a 'tup' is used to apply a load to the centerline

opposite the notch. The DWTT specimen had a width, W, of $76.2 \mathrm{~mm}$, span between anvils, S, of $250.4 \mathrm{~mm}$, thickness, B, of $13.7 \mathrm{~mm}$. Typical notch geometry can be seen in Figure 2.6 (b) (Xu and Tyson, 2011b). The depth of the notch can vary from specimen to specimen depending on the researcher's preference and can range from 10-38 mm.

An experimental quasi-static DWTT was performed by Xu from CANMET-MTL (Xu, 2010a), and load vs. load-line displacement data was provided to verify the modeling performed in this work. The experiment performed by $\mathrm{Xu}$ had an original notch depth, a, of $14.7 \mathrm{~mm}$. The specimen was a C2 (X70) steel, and was flattened from a pipeline section. The specimen was machined from the pipe so the crack would grow in what was the axial direction of the pipe.

\subsubsection{Experimental Setup and Data Collection}

The quasi-static test set-up for measuring CTOA from a DWTT specimen can be seen in Figure 2.7 (Xu et al, 2010b). Along the bottom are the two anvils used to support the specimen. The load is applied to the center of the specimen at the top by the 'tup'. There are anti-buckling plates to ensure the specimen remains in-plane.

The applied load is determined by measuring the reaction force due to the displacement applied by the 'tup'. The load-line displacement is measured as the displacement of the loading jig. This is a potential source of error however, as there will be elastic compression in the fixture, and 
local indentation in the specimen. It is possible to overcome these errors by collecting the loadline displacement for compliance, as recommended by ASTM E1820 (2001). Compliance is defined as the ratio of displacement increment to load increment. The compliance can be expressed as a function of the crack length:

$$
\mathrm{C}=\frac{L L D}{P}=\frac{Y^{2}}{B_{e} E^{\prime}}\left[A_{o}+A_{1}\left(\frac{a}{W}\right)+A_{2}\left(\frac{a}{W}\right)^{2}+A_{3}\left(\frac{a}{W}\right)^{3}+A_{4}\left(\frac{a}{W}\right)^{4}+A_{5}\left(\frac{a}{W}\right)^{5}\right]
$$

Where for a $\mathrm{SE}(\mathrm{B})$ specimen, $\mathrm{Y}=\mathrm{S} /(\mathrm{W}-\mathrm{a}), A_{o}=1.193, A_{1}=-1.980, A_{2}=4.478, A_{3}=$ $-4.333, A_{4}=1.739$, and $A_{5}=0$. Additionally,

$$
\begin{gathered}
B_{e}=B-\frac{\left(B-B_{N}\right)^{2}}{B} \\
E^{\prime}=\frac{E}{\left(1-v^{2}\right)}
\end{gathered}
$$

In the case of the experimental data for the $\mathrm{C} 2$ material, as provided by $\mathrm{Xu}(2010 \mathrm{a}), \mathrm{B}_{\mathrm{N}}=\mathrm{B}$, since no side grooves are present. This means that $\mathrm{B}_{\mathrm{e}}=\mathrm{B}=13.7 \mathrm{~mm}$. Additionally, $\mathrm{E}^{\prime}=214.73$ $\mathrm{GPa}$, since $\mathrm{E}=195.4 \mathrm{GPa}$, and $v=0.3$. Furthermore, $\mathrm{Y}=(250.4 \mathrm{~mm}) /(76.2 \mathrm{~mm}-14.6 \mathrm{~mm})=$ 4.065. These values lead to a compliance of $5.332 \times 10^{-3} \mathrm{~mm} / \mathrm{kN}$, or a desired elastic slope on the graph of $187.6 \mathrm{kN} / \mathrm{mm}$. The given LLD data was then corrected to meet this slope as follows:

$$
L L D^{\text {corrected }}=L L D^{\text {measured }}-\frac{P}{\text { Elastic Error Constant }}-\text { offset error }
$$

In this case, the elastic error constant used was $90 \mathrm{kN}$, and the offset error was $0.2439 \mathrm{~mm}$. The corrected load vs. LLD data is presented in Table 2.1. The data is then plotted in Figure 2.8. 


\subsubsection{Measuring the Crack Tip Opening Angle}

There have been many experimental techniques proposed for measuring the CTOA, each with their own advantages and drawbacks. The methods that will be discussed here are direct or optical measurement and the simplified single-specimen method. Theory relating the CTOA to other toughness parameters will also be discussed.

\section{Measuring CTOA Using Optical Techniques}

The most common optical technique for measuring the CTOA is direct surface measurement. It is typically difficult to accurately identify the crack tip when performing direct surface measurement so a method has been developed that does not require identification of the exact crack tip. The method requires finding the average of 3-5 four point measurements of CTOA by determining the angle between the lines created by joining two points on the upper and lower surfaces. A set of points at an original reference is used with 3-5 other pairs of points all within $0.5-1.5 \mathrm{~mm}$ behind the estimated crack tip. Figure 2.9 demonstrates the technique. This averaging technique helps to minimize discrepancies in results when measuring the CTOA for wavy crack flanks, as seen in Figure 2.10 (Xu et al, 2008).

It is agreed that the CTOA measured by direct surface measurement is higher than the CTOA that would be at the mid-plane of the specimen. This is due to the three-dimensional nature of the crack propagation (crack tunnelling effect, flat-to-shear transition, etc.).

\section{Measuring CTOA with the Simplified Single-Specimen Method}

A method for calculating the CTOA from DWTT specimens has been proposed by Xu et al (2007). It is called the simplified single-specimen method (S-SSM), and was been adapted from the single-specimen method proposed by Martinelli and Venzi (1996). The S-SSM proposes that the CTOA for a DWTT specimen can be calculated as follows: 


$$
C T O A=2 \cdot \tan ^{-1}\left[\frac{-4 r^{*}}{S} \cdot \frac{1}{\frac{d \ln P}{d y}}\right]
$$

Where $\mathrm{r}^{*}$ is the rotation factor, $\mathrm{S}$ is the span between the two load points, $\mathrm{P}$ is the applied load, and $\mathrm{y}$ is the load-line displacement (LLD). These parameters are defined in Figure 2.6 (a). Therefore, the CTOA can be calculated from only the slope of the LnP vs. LLD curve, in addition to values for the rotation factor and the span. The specimen is assumed to rotate around a point at a distance of $r^{*} \cdot b$ away from the crack-tip, where $\mathrm{b}$ is the length of the remaining ligament. The value of the rotation factor is recommended to be 0.57 for high strength steels, and 0.54 for low strength steels Xu et al $(2007,2008,2009,2011)$.

Both the optical and S-SSM were used to measure the CTOA during an experimental dropweight tear test of a C2 steel, performed by Xu et al (2010b). The results published by Xu et al (2010b) will be compared to the CTOAs obtained from the finite element simulations for the C2 steels in Chapter 5. 


\begin{tabular}{|c|c|c|c|c|c|c|c|}
\hline $\operatorname{LLD}(\mathrm{mm})$ & $P(k N)$ & $\operatorname{LLD}(\mathrm{mm})$ & $P(k N)$ & $\operatorname{LLD}(\mathrm{mm})$ & $\mathrm{P}(\mathrm{kN})$ & $\operatorname{LLD}(\mathrm{mm})$ & $P(k N)$ \\
\hline 0.0 & 10.5 & 4.0 & 213.6 & 16.0 & 163.7 & 28.0 & 65.0 \\
\hline 0.1 & 23.4 & 4.5 & 217.1 & 16.5 & 158.8 & 28.5 & 62.5 \\
\hline 0.2 & 41.2 & 5.0 & 220.2 & 17.0 & 153.7 & 29.0 & 60.0 \\
\hline 0.3 & 62.2 & 5.5 & 222.8 & 17.5 & 148.3 & 29.5 & 57.7 \\
\hline 0.4 & 82.3 & 6.0 & 222.7 & 18.0 & 144.0 & 30.0 & 55.6 \\
\hline 0.5 & 101.4 & 6.5 & 222.8 & 18.5 & 139.1 & 30.5 & 53.5 \\
\hline 0.6 & 119.4 & 7.0 & 221.1 & 19.0 & 134.7 & 31.0 & 51.0 \\
\hline 0.7 & 136.2 & 7.5 & 219.0 & 19.5 & 129.1 & 31.5 & 48.5 \\
\hline 0.8 & 150.0 & 8.0 & 217.7 & 20.0 & 123.9 & 32.0 & 46.7 \\
\hline 0.9 & 161.2 & 8.5 & 215.4 & 20.5 & 119.6 & 32.5 & 45.4 \\
\hline 1.0 & 169.3 & 9.0 & 212.1 & 21.0 & 115.7 & 33.0 & 44.0 \\
\hline 1.1 & 175.6 & 9.5 & 209.4 & 21.5 & 111.6 & 33.5 & 42.8 \\
\hline 1.2 & 180.2 & 10.0 & 206.1 & 22.0 & 107.8 & 34.0 & 41.4 \\
\hline 1.3 & 183.6 & 10.5 & 202.3 & 22.5 & 102.9 & 34.5 & 40.3 \\
\hline 1.4 & 186.1 & 11.0 & 198.9 & 23.0 & 98.5 & 35.0 & 39.3 \\
\hline 1.5 & 188.4 & 11.5 & 196.4 & 23.5 & 94.8 & 35.5 & 38.2 \\
\hline 1.6 & 190.3 & 12.0 & 192.3 & 24.0 & 90.7 & 36.0 & 37.2 \\
\hline 1.7 & 191.9 & 12.5 & 189.5 & 24.5 & 87.1 & 36.5 & 36.2 \\
\hline 1.8 & 193.5 & 13.0 & 186.6 & 25.0 & 83.6 & 37.0 & 34.5 \\
\hline 1.9 & 194.8 & 13.5 & 183.1 & 25.5 & 80.6 & 37.5 & 33.5 \\
\hline 2.0 & 196.0 & 14.0 & 180.6 & 26.0 & 77.1 & 38.0 & 32.6 \\
\hline 2.5 & 201.3 & 14.5 & 175.9 & 26.5 & 73.9 & 38.5 & 31.8 \\
\hline 3.0 & 205.8 & 15.0 & 171.2 & 27.0 & 70.8 & 39.0 & 31.0 \\
\hline 3.5 & 209.9 & 15.5 & 168.0 & 27.5 & 67.9 & 39.5 & 30.3 \\
\hline & & & & & & 40.0 & 29.5 \\
\hline
\end{tabular}

Table 2.1: P vs. LLD Data from CANMET - C2 Steel - Corrected for Compliance

$$
(\mathrm{Xu}, 2010 \mathrm{a})
$$




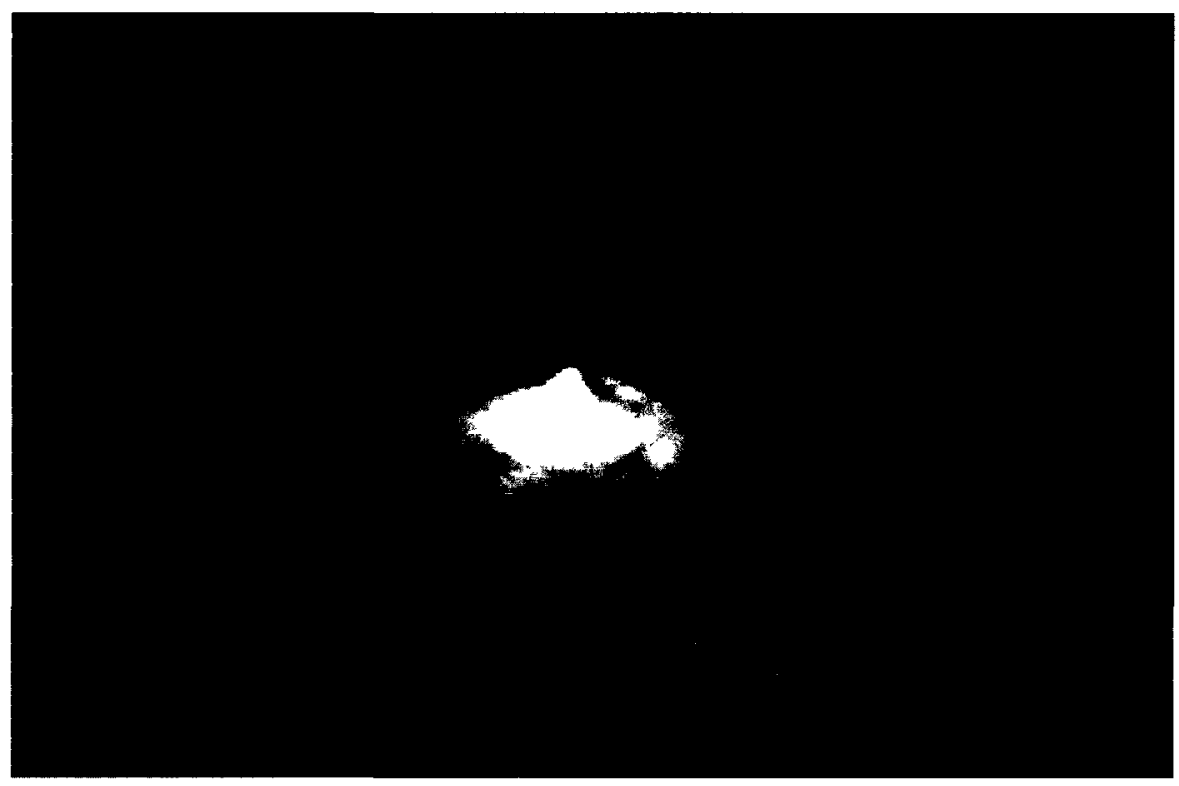

Figure 2.1: Partially Fractured Charpy Sample (post test) (Xu et al, 2004)
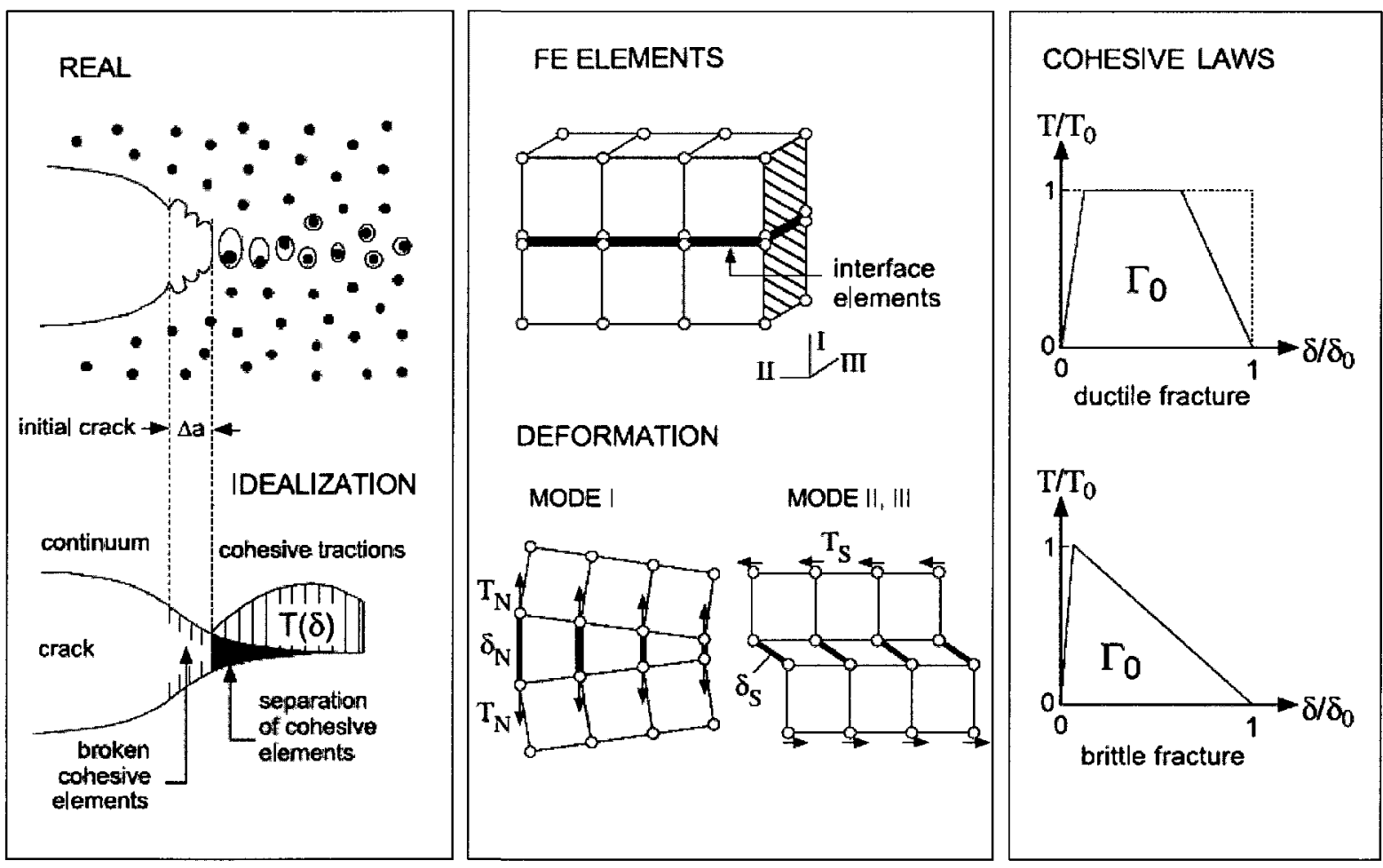

Figure 2.2: Representation of the Ductile Failure Process by CZM (Cornec, 2003) 


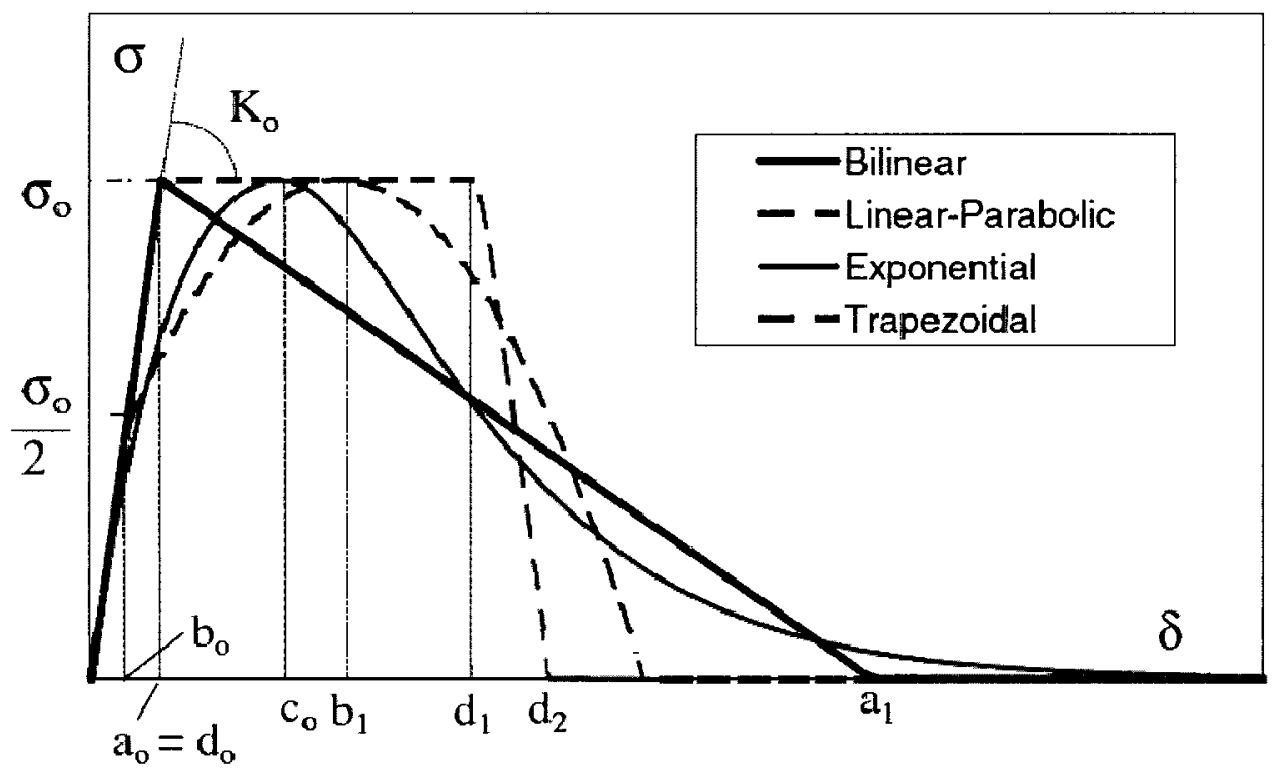

Figure 2.3: Four Examples of Various Shapes of TS laws (Alfano, 2006) $\left(\sigma_{0}=T_{0}=\right.$ peak traction, $a_{1}=\delta_{c}=$ critical separation, for bilinear law $)$

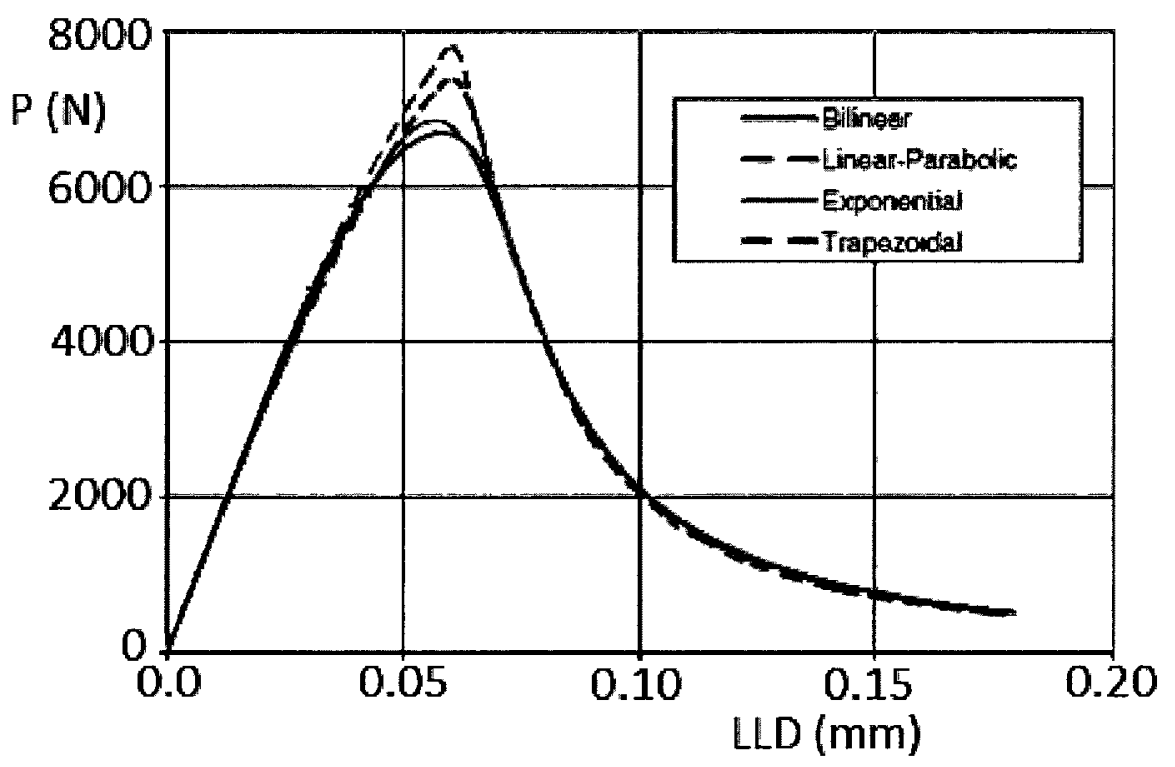

Figure 2.4: $\mathrm{C}(\mathrm{T})$ Test: P vs. LLD Curves for Various TS law Shapes (Alfano, 2006) 


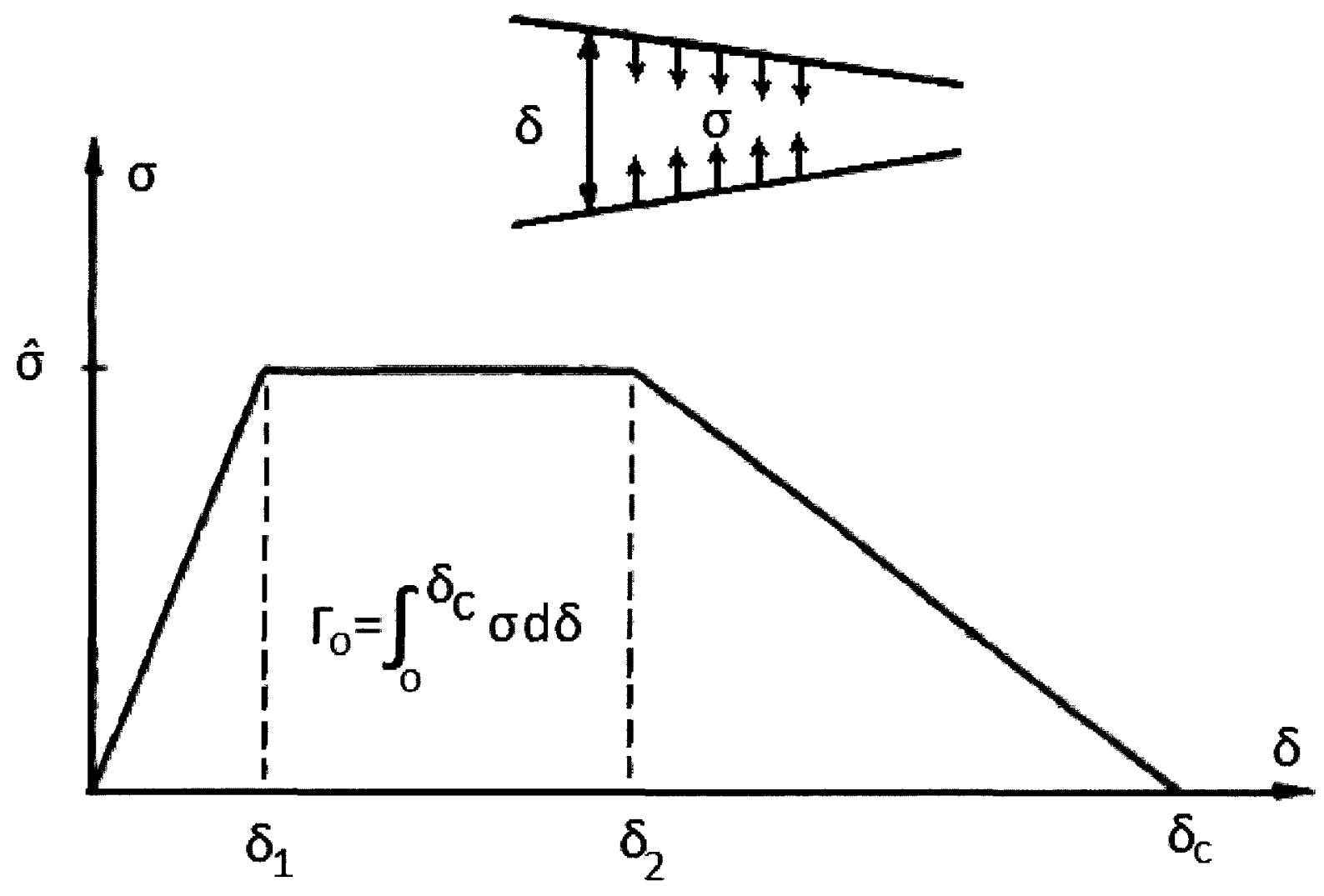

Figure 2.5: Trapezoidal Traction-Separation Law (Tvergaard and Huchinson, 1992)

$$
\left(\widehat{\sigma} \equiv T_{o}\right)
$$




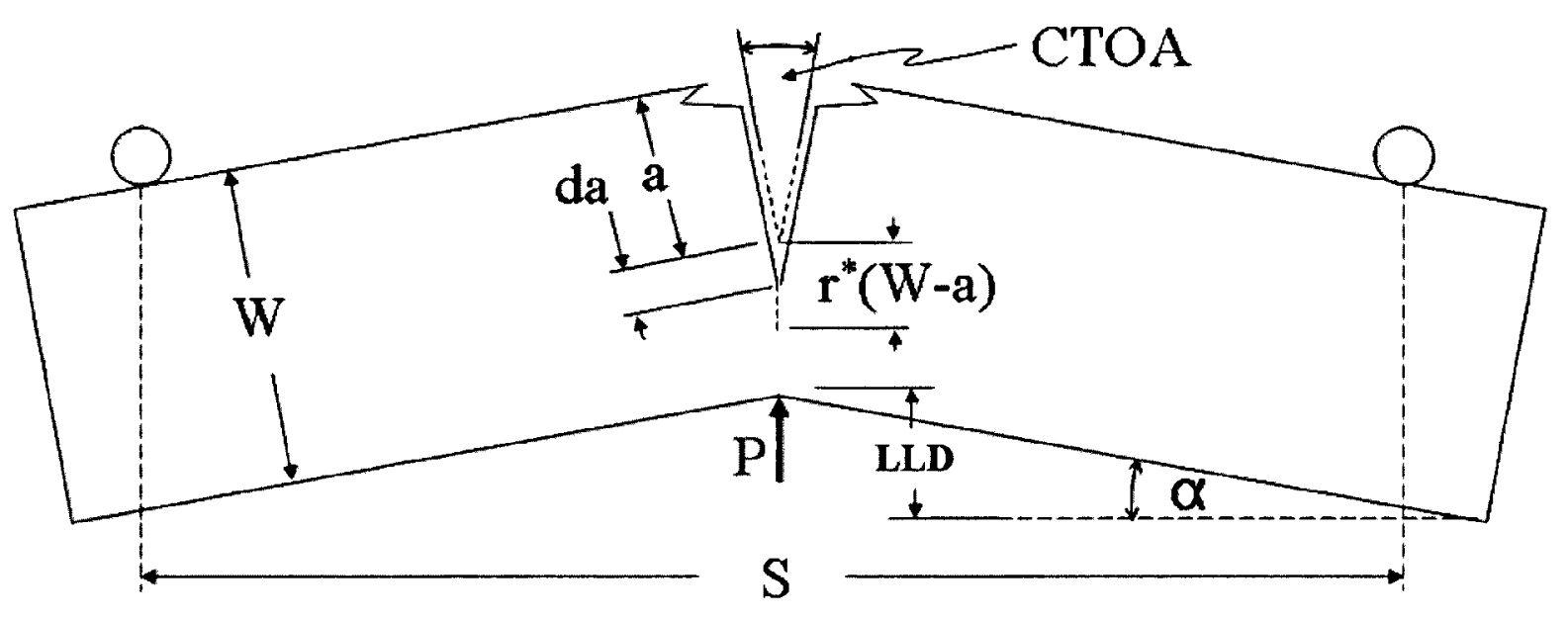

(a) Full Specimen (Xu et al, 2011a)

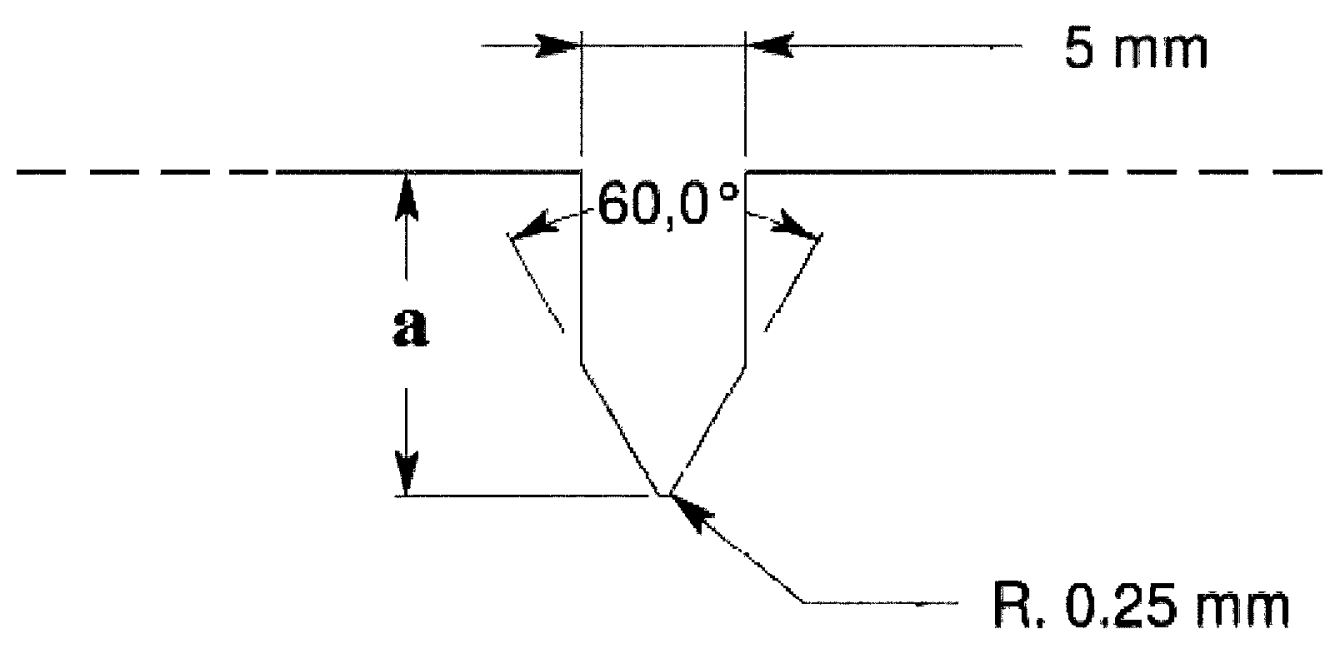

(b) Notch Geometry (Xu and Tyson, 2011b)

Figure 2.6: DWTT Geometry (a) Full Specimen, (b) Notch Geometry (cut-view) 


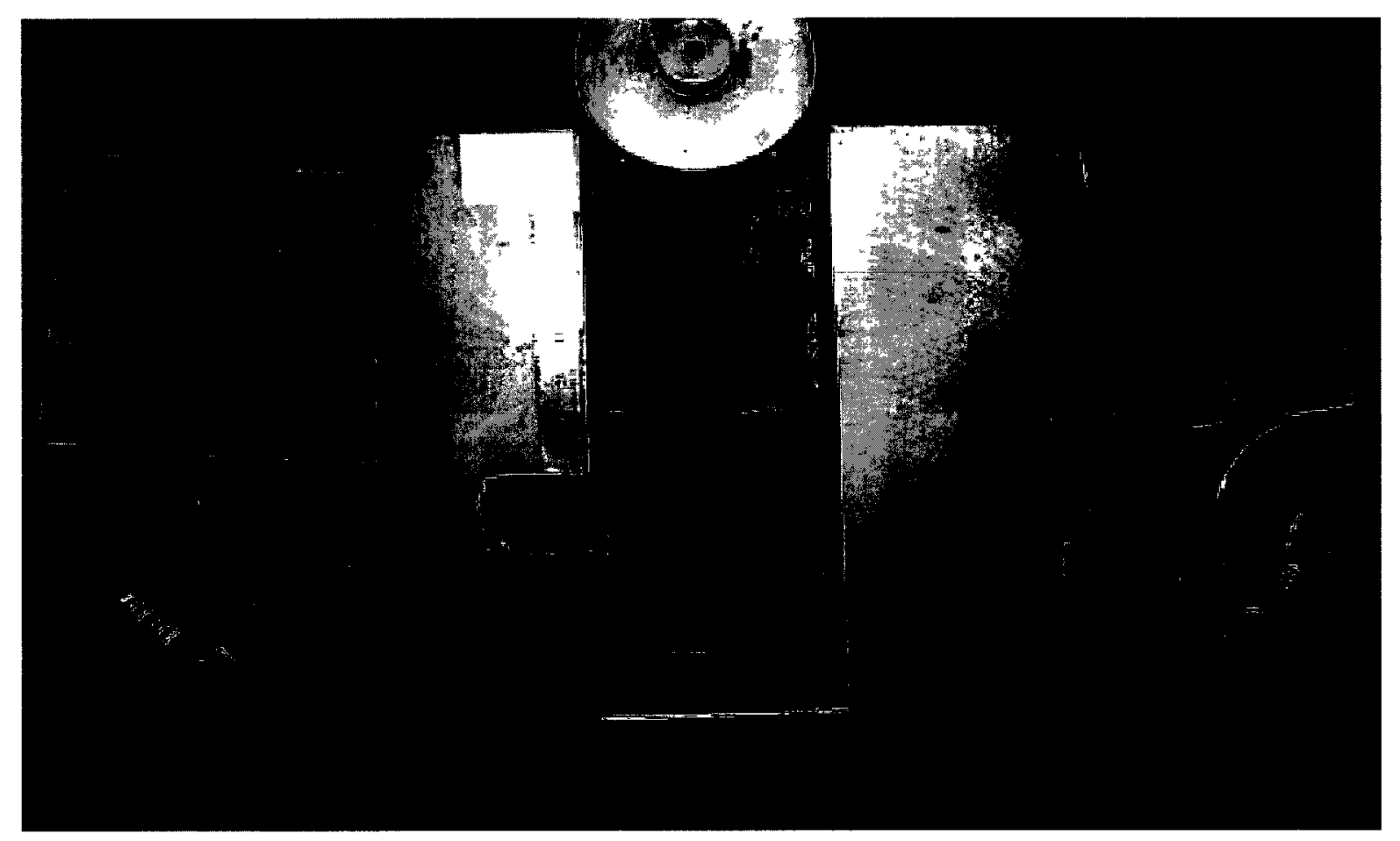

Figure 2.7: Experimental DWTT Set-up (Xu et al, 2010b) 


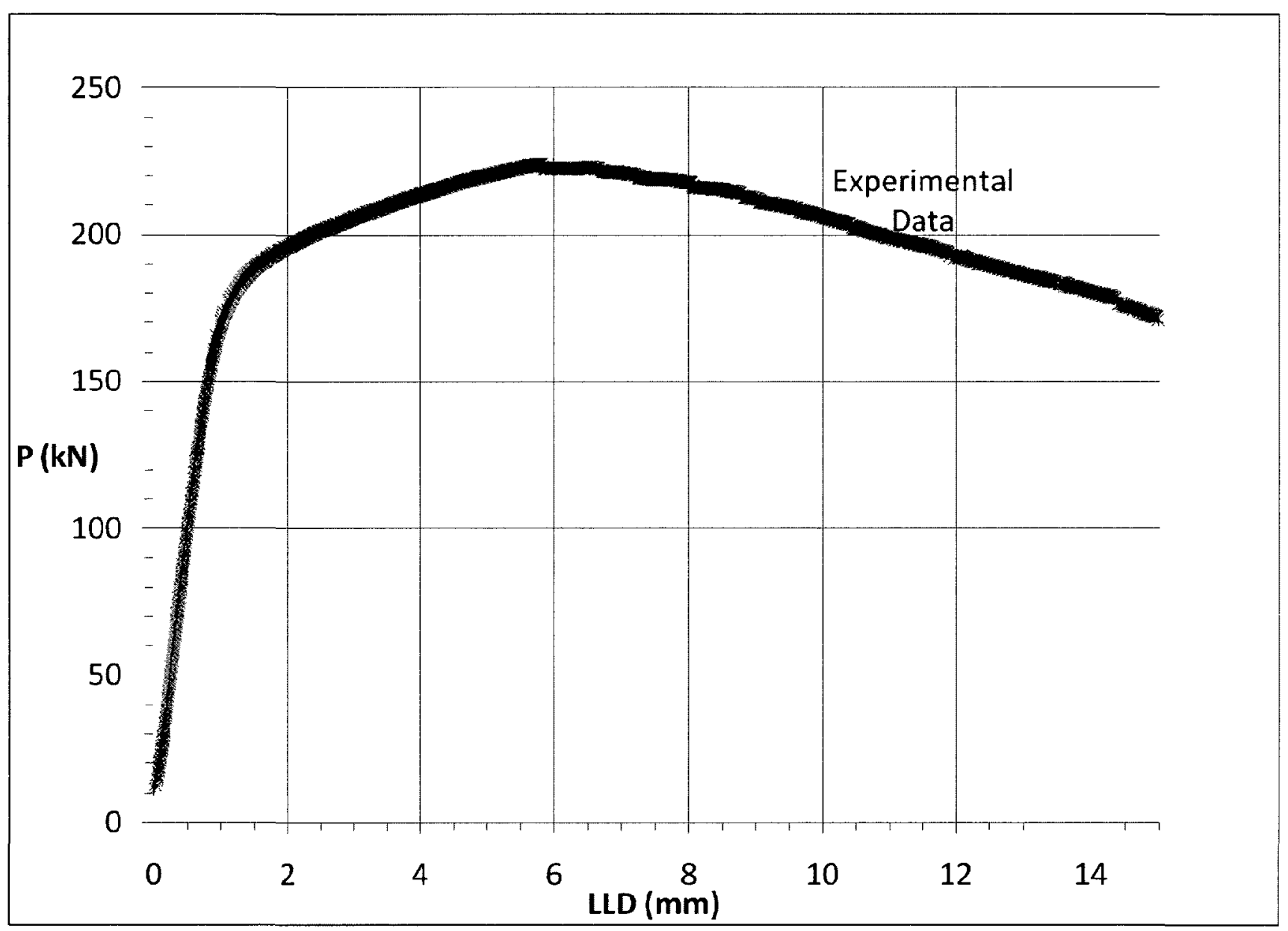

Figure 2.8: P vs. LLD Data for C2 steel - Corrected for Compliance (Xu, 2010a) 


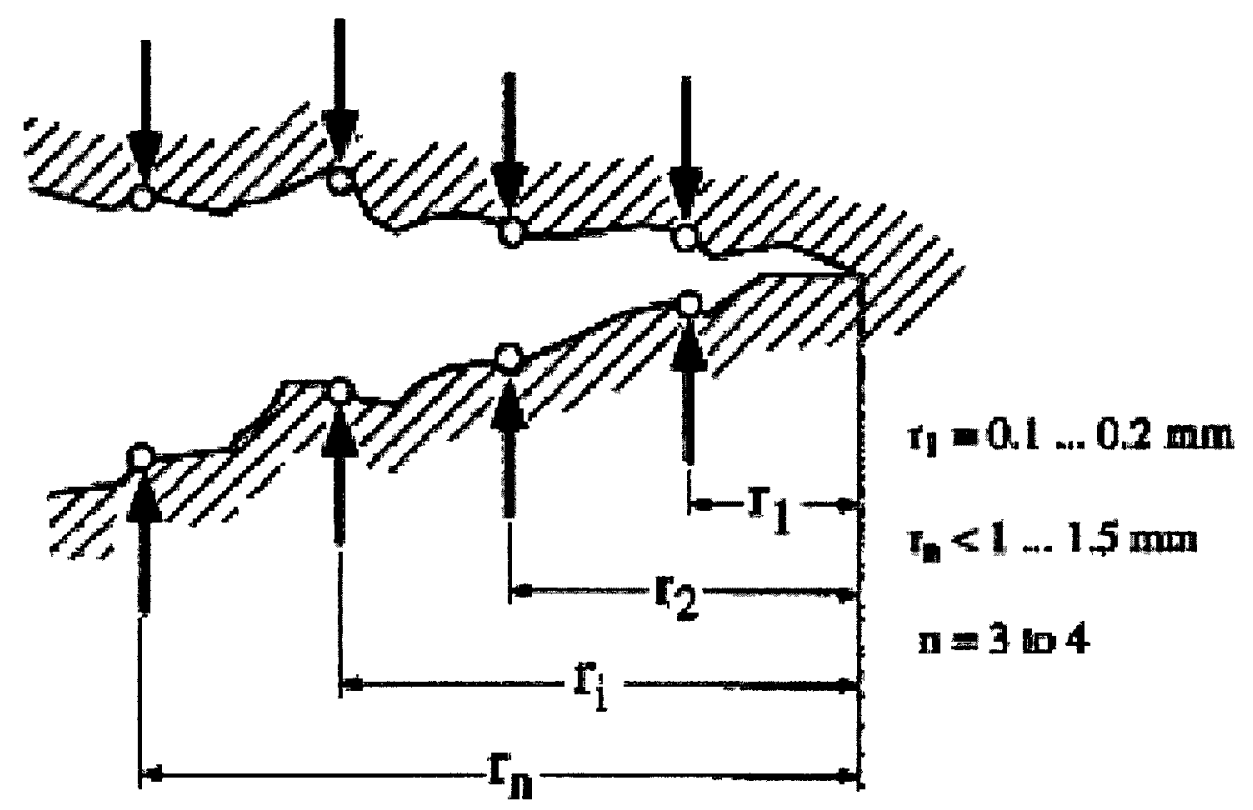

Figure 2.9: Surface Measurement of CTOA 


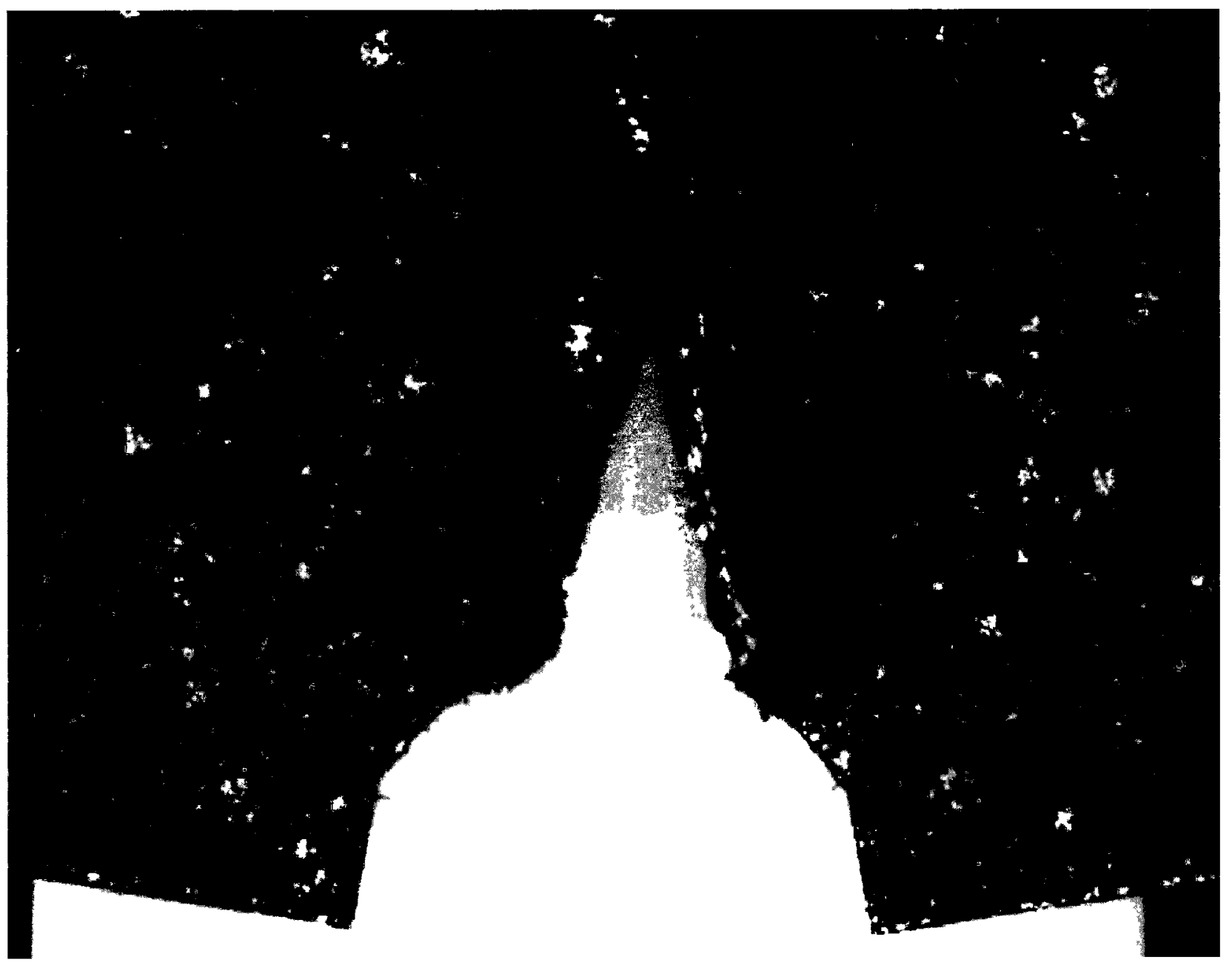

Figure 2.10: Slow-rate-tested X70 Pipe Sample at a Crack Length of $30 \mathrm{~mm}$ Showing Wavy Crack Flanks (Xu et al, 2008) 


\section{Chapter 3}

\section{Finite Element Implementation of Cohesive Zone Modeling}

This chapter will discuss the implementation of cohesive zone modeling (CZM) in Abaqus (2008) using finite element techniques. The process of defining model parameters will be outlined and analysis of a unit cell is performed to validate the theory.

\subsection{Finite Element Implementation of CZM}

A cohesive element is a type of finite element that has a built-in damage evolution law. The cohesive element will have an elastic response until subjected to certain maximum damage criteria. Once the damage criterion has been met, the element stiffness will degrade based on some damage evolution law, as defined by the user. A cohesive zone model uses a layer of cohesive elements to model separation of two parts. To predict fracture, it is possible to imagine an infinitesimally thin layer of cohesive elements along the crack path that fail sequentially as the crack grows.

\subsubsection{Cohesive Zone Model}

It is possible to use Abaqus to perform cohesive zone modeling. Various cohesive element definitions exist in both Abaqus/Standard and Abaqus/Explicit for both two-dimensional (COH2D4, COH2D4P) and three-dimensional modeling (COH3D6, COH3D8). For twodimensional modeling of crack growth it is appropriate to use the $\mathrm{COH} 2 \mathrm{D} 4$ element. Node numbering conventions, integration point locations, and the thickness direction for the COH2D4 element can be seen in Figure 3.1 (Abaqus, 2008). 
Typical uses of cohesive elements in Abaqus are continuum-based modeling, traction-separationbased modeling, and modeling of gaskets or laterally unconstrained adhesive patches. Continuum-based modeling would be used for situations where two parts are bonded together by an adhesive layer of some finite thickness. Traction-separation-based modeling would be used for situations where the interface between two parts can be considered to have zero thickness. The response of gaskets can be modeled assuming a uniaxial stress state and requires only macroscopic properties like material strength and stiffness. When modeling crack growth along a known crack path, it is recommended to use a traction-separation-based approach using a layer of cohesive elements along the crack path of close to zero-thickness (Abaqus, 2008).

Figure 3.2 (Abaqus, 2008) shows a typical design for a cohesive zone model. The model is divided into three separate parts, each meshed independently and constrained to act as one part. A thin layer of cohesive elements is connected to the surrounding parts using tie constraints that cause the entire assembly to act as one structure. Parts 1 and 2 are meshed using continuum elements with a built in elastic-plastic response. This type of model would allow a crack to grow through the cohesive layer as the cohesive elements fail. A similar approach will be used and described in Chapters 4 and 5 to predict crack growth in small-scale yielding and DWTT models. Further meshing considerations will be discussed in Chapter 4 .

\subsubsection{Traction-Separation-Based Modeling}

Traction-separation-based modeling allows the user to define a progressive degradation law that defines the stiffness of cohesive element based on some damage evolution process which is controlled by the element's built in damage variable, D. A typical bilinear traction-separation (TS) law is defined in Figure 3.3.

There are three important values on the traction-separation curve that must be defined: 
- $t_{i}^{0}$ is the peak traction, or maximum normal stress that the element can resist without damage. Damage initiates at $\sigma_{l}=t_{i}^{0}$

- $\delta_{i}^{0}$ is the separation that causes damage to initiate, damage $\mathrm{D}=0$

- $\delta_{\imath}^{f}$ is the separation where damage $\mathrm{D}=1$, and the element's ability to transfer loading is completely eliminated, $\left(t_{t}=0\right.$ when $\delta_{i} \geq \delta_{l}^{f}$ and $\left.D \geq 1\right)$

The built-in traction-separation models in Abaqus (2008) assume an initial linear elastic portion, followed by initiation of damage at some maximum traction and a progressive degradation of stiffness based on a defined damage evolution process.

\section{Linear Elastic Behaviour}

The initial elastic behaviour of a cohesive element is defined as

$$
t=\left\{\begin{array}{l}
t_{n} \\
t_{s} \\
t_{t}
\end{array}\right\}=\left[\begin{array}{lll}
K_{n n} & K_{n s} & K_{n t} \\
K_{n s} & K_{s s} & K_{s t} \\
K_{n t} & K_{s t} & K_{t t}
\end{array}\right]\left\{\begin{array}{c}
\varepsilon_{n} \\
\varepsilon_{s} \\
\varepsilon_{t}
\end{array}\right\}=K \varepsilon
$$

where $t$ is the element's traction, $K$ is the stiffness, and $\varepsilon$ is strain. The subscripts $n, s$, and $t$ represent the normal, shear and tangential directions respectively. Nominal strains are defined as

$$
\varepsilon_{l}=\frac{\delta_{l}}{T_{o}}, i=\mathrm{n}, \mathrm{s}, \mathrm{t}
$$

where $\delta_{i}$ represents the element separation, and $\mathrm{T}_{\mathrm{o}}$ represents the initial thickness of the cohesive element, usually defined as equal to 1.0 regardless of element geometry so that the strains in each direction are equal to the separations in that direction.

When using uncoupled traction-separation laws, as will always be the case in this thesis, it is only required to define $\mathrm{K}_{\mathrm{nn}}, \mathrm{K}_{\mathrm{ss}}$, and $\mathrm{K}_{\mathrm{tt}}$ as the strain in one direction has no effect on the strain in the others for the cohesive elements $\left(K_{n s}, K_{n t}, K_{s t}=0\right)$ 


\section{Damage Initiation}

Damage initiation occurs at a point where the maximum traction or maximum separation of the element has been reached. Abaqus (2008) has four methods for defining the maximum damage of a TS law. These are the maximum nominal stress criterion (Maxs), the maximum nominal strain criterion (Maxe), the quadratic nominal stress criterion (Quads) and the quadratic nominal strain criterion (Quade). The maximum nominal stress criterion has been used exclusively in this thesis. This means that damage initiates when:

$$
\max \left\{\frac{\left\langle t_{n}\right\rangle}{t_{n}^{0}}, \frac{t_{s}}{t_{s}^{0}}, \frac{t_{t}}{t_{t}^{0}}\right\}=1
$$

The angular brackets on $t_{n}$ indicate that only positive traction (tension) is considered, and that compression does not cause damage to initiate or grow.

\section{Damage Evolution}

The selected damage evolution law is what defines the portion of the traction-separation curve between the damage initiation at $\delta_{i}^{0}$ and complete element failure at $\delta_{i}^{f}$. Abaqus (2008) controls this evolution process by using a scalar damage variable, D. This damage variable takes into account one or more damage mechanisms as defined by the user, and represents the overall damage in the material. The actual stress components in a cohesive element are then defined as

$$
\begin{aligned}
& t_{n}=\left\{\begin{array}{cc}
(1-D) \bar{t}_{n}, & \bar{t}_{n} \geq 0 \\
\bar{t}_{n}, & \bar{t}_{n}<0
\end{array}\right. \text { (no damage from compressive loading) } \\
& t_{s}=(t-D) \bar{t}_{s} \\
& t_{t}=(t-D) \bar{t}_{t}
\end{aligned}
$$

where tractions $\bar{t}_{n}, \bar{t}_{s}, \bar{t}_{t}$ are pre-damage values predicted by equation (3.1).

Abaqus allows damage evolution to be defined based on effective displacement, energy, or by directly defining damage variable, $\mathrm{D}$, as a tabular function. 
When defining the damage evolution based on effective displacement, Abaqus requires the user to define the quantity $\delta_{m}^{f}-\delta_{m}^{0}$, or the effective displacement at failure minus the effective displacement at damage initiation. The softening law between $\delta_{m}^{o}$ and $\delta_{m}^{f}$ can then be chosen to be linear, exponential, or with a specified damage, D defined as a tabular function of the effective displacement after initiation. The effective displacement, $\delta_{m}$, is defined as:

$$
\delta_{m}=\sqrt{\left\langle\delta_{n}\right\rangle^{2}+\delta_{s}^{2}+\delta_{t}^{2}}
$$

Where the brackets on $\left\langle\delta_{n}\right\rangle$ mean that only positive values of $\delta_{n}$ are considered. If a linear softening law is chosen such as in Figure 3.3, then the damage variable is defined as follows:

$$
D=\frac{\delta_{m}^{f}\left(\delta_{m}^{\max }-\delta_{m}^{0}\right)}{\delta_{m}^{\max }\left(\delta_{m}^{f}-\delta_{m}^{0}\right)}
$$

where $\delta_{m}^{\max }$ refers to the maximum effective displacement attained by the cohesive element throughout its complete loading history. It is seen that damage variable D increases linearly from 0 to 1 between $\delta_{m}^{0}$ and $\delta_{m}^{f}$. While the damage variable continues increasing past a separation of $\delta_{m}^{f}$, it no longer matters for the macroscopic response of the model as the cohesive element will no longer be able to transfer any load.

For this work however, the traction-separation laws are all defined in terms of the fracture energy, $\Gamma_{0}$, (the area under the TS curve) to better relate them to fracture mechanics. The same equation defined by equation (3.6) applies still, but now the effective displacement at failure is defined by the equation:

$$
\delta_{m}^{f}=\frac{2 \cdot \Gamma_{o}}{T_{e f f}^{o}}
$$


where $T_{e f f}^{o}$ is the effective traction at the point of damage initiation (the user input Maxs).

\subsubsection{Determining Model Parameters}

\section{Cohesive Parameters}

For this work, all the traction-separation laws used are bilinear, with damage evolution defined by the fracture energy. This was done in part because Abaqus version 6.8-2 does not have a built-in method for defining trapezoidal TS laws. The bilinear laws were thus chosen as a first approach to model fracture propagation of $\mathrm{C} 2$ steels. The bilinear shape was chosen for its simplicity, its numerical stability, and evidence of its predictive ability from work by Alfano (2006) and Chandra et al (2002). Alfano has concluded that the trapezoidal law, first used by Tvergaard and Hutchinson (1992), was the least numerically stable and the farthest away from achieving convergence to the exact solution of the four shapes investigated. Therefore to fully define the traction-separation response of the cohesive elements in Abaqus/Explicit, the following parameters must be defined:

- Mass density $(\rho)$

- $\quad$ Elastic stiffnesses $\left(\mathrm{K}_{\mathrm{nn}}, \mathrm{K}_{\mathrm{ss}}\right.$, and $\left.\mathrm{K}_{\mathrm{tt}}\right)$

- The maximum nominal stress in the normal, shear, and tangential directions $\left(\mathrm{t}_{\mathrm{n}}^{0}, \mathrm{t}_{\mathrm{s}}^{0}, \mathrm{t}_{\mathrm{t}}^{0}\right)$

- The fracture energy $\left(\Gamma_{0}\right)$

The simulations performed in this work are all for high strength steels, and the density has been selected accordingly as $7.85 \times 10^{-6} \mathrm{~kg} / \mathrm{mm}^{3}$.

The elastic stiffness parameter, $\mathrm{K}_{\mathrm{nn}}$, is treated as a penalty parameter. In order for the cohesive layer to have negligible effect on the total stiffness of the model, it is desirable to have infinite cohesive stiffness. While this isn't realistic for numerical simulations, it is still desirable to have a cohesive stiffness, $K_{n n}$, that is much higher than the elastic-plastic stiffness, E, of the surrounding elastic-plastic elements. A value of 10,000,000 $\mathrm{MPa}$ has been chosen for $\mathrm{K}_{\mathrm{nn}}$ in most of the TS laws used in this work. Through trial and error this value has been determined to be stiff enough to limit the macroscopic effect of the cohesive layer's stiffness, and small enough 
to maintain a reasonable time-step for most simulations presented here. For this work, just as $\mathrm{G}=$ $\mathrm{E} / 2(1+\mathrm{v}), \mathrm{K}_{\mathrm{ss}}$ and $\mathrm{K}_{\mathrm{tt}}$ are set equal to $\mathrm{K}_{\mathrm{nn}} / 2(1+v)$. Poisson's ratio, $v$, is set as 0.3 , as appropriate for steel. Therefore $\mathrm{K}_{\mathrm{ss}}$ and $\mathrm{K}_{\mathrm{tt}}$ are both equal to 3,846,150 MPa for most TS laws in this work.

The maximum nominal stress varies for each simulation in this work, but is always a multiple of the surrounding elastic-plastic material's yield strength. Typical values for $t_{n}^{0}$ range from $(3.0-$ 4.0) $\cdot \sigma_{\mathrm{y}}$. For all cases $\mathrm{t}_{\mathrm{s}}^{0}$ and $\mathrm{t}_{\mathrm{t}}^{0}$ are set equal to $0.75 \cdot \mathrm{t}_{\mathrm{n}}^{0}$, as the actual values are unknown, and shear strength is typically equal to approximately $3 / 4$ of the yield strength. Note, since the simulations performed in this work all involve only mode-I loading and damage initiation always occurs due to the $\left\langle t_{n}\right\rangle / t_{n}^{0}$ term of equation (3.3), these values of $t_{s}^{0}$ and $t_{t}^{0}$ have no effect on the simulation results.

The fracture energy, $\Gamma_{0}$, is a function of the peak traction and the maximum desired separation. Calibration of this parameter is further discussed in Chapters 4 and 5. Typical values for this work range from 6-18 $\mathrm{MPa} \cdot \mathrm{mm}$.

\section{Continuum Material Parameters}

Two different steels are considered in this work. The first is defined by Tvergaard and Hutchinson (1992) as an ideal ductile steel that follows a true-stress-logarithmic strain curve specified by:

$$
\begin{gathered}
\varepsilon=\frac{\sigma}{E} \quad \sigma \leq \sigma_{y}, \\
\varepsilon=\left(\frac{\sigma_{y}}{E}\right)\left(\frac{\sigma}{\sigma_{y}}\right)^{1 / N} \quad \sigma \leq \sigma_{y},
\end{gathered}
$$

where $\mathrm{N}=0.1, \sigma_{\mathrm{y}} / \mathrm{E}=0.003$, and $v=0.3$. Picking a typical high-strength steel yield stress of 600 MPa gives a value of 200,000 MPa for Young's Modulus, well within the expected range for ductile steels. This material shall hereafter be referred to as the TH material. This TH material can be input into Abaqus with the given elastic properties, and a stress vs. plastic strain tabular function as given in Table 3.1. The elastic-plastic curve is found in Figure 3.4. The tabular values 
are obtained based on Equation 3.8. It is necessary to use the tabular function approximation of stress vs. plastic strain as it is not possible to define the plastic deformation with an equation in Abaqus/Explicit.

The other steel used is from work done by Xu et al $(2010 \mathrm{a}, \mathrm{b})$ on DWTT specimens machined from pipelines. It is a C2 steel, with a yield strength of $576 \mathrm{MPa}$ a Young's modulus of 195,400 $\mathrm{MPa}$ and a Poisson's ratio of 0.3 . Chemical composition of this $\mathrm{C} 2$ steel can be found in Table 3.2, and material properties can be found in Table 3.3. A true stress-strain curve provided by S. $\mathrm{Xu}$ for the $\mathrm{C} 2$ material is given in Figure 3.5 (Xu, 2010a). The plastic strain data entered into Abaqus to model this curve is given in Table 3.4. This material shall hereafter be referred to as the $\mathrm{C} 2$ material.

\subsection{Unit Cell Analysis}

\section{TH Materials}

A unit cell analysis was carried out to verify how cohesive elements function in Abaqus/Explicit. For the TH materials, a single cohesive element was created with a length of $0.03 \mathrm{~mm}$ and thickness of $0.01 \mathrm{~mm}$. The nominal thickness defined in Abaqus was left as the default 1.0. This allows direct comparison between the separation and strain of the cohesive elements. The bottom surface of the cohesive element was subject to a fixed constraint in the y-direction, with node 1 also fixed in the $\mathrm{x}$-direction. A ramped displacement was then applied to the top surface that increased from $0-0.007 \mathrm{~mm}$ as the simulation time increased from $0-1 \mathrm{~s}$. The geometry and boundary conditions of the element are shown in Figure 3.6.

Tvergaard and Hutchinson (1992) defined four separate cases for their trapezoidal TS law, each with a different peak traction. The maximum separation remained constant for each case, so the fracture energy also varied from case to case. The maximum tractions were equal to $3.0 \cdot \sigma_{\mathrm{y}}$, 
$3.5 \cdot \sigma_{\mathrm{y}}, 3.6 \cdot \sigma_{\mathrm{y}}$, and $3.75 \cdot \sigma_{\mathrm{y}}$ for cases $1-4$ respectively. If a yield strength of $600 \mathrm{MPa}$ is assumed as decided earlier, this leads to maximum normal stresses of $1800,2100,2160$, and $2250 \mathrm{MPa}$ for cases $1-4$ respectively.

For each case, $\delta_{c} / \Delta_{o}=0.1, \delta_{1} / \delta_{c}=0.15$, and $\delta_{2} / \delta_{c}=0.5$, where $\delta_{1}, \delta_{2}$, and $\delta_{c}$ are as defined in Figure 2.5, and $\Delta_{o}$ is the length of the smallest elastic-plastic element in the small-scale yielding mesh model that will be outlined in Chapter 4 . As will be seen later, for the TH materials, $\Delta_{o}=0.1 \mathrm{~mm}$. That means that $\delta_{1}=0.001, \delta_{2}=0.005$ and $\delta_{c}=0.01 \mathrm{~mm}$. Inserting these values along with the peak traction for each case into Equation 2.5 allows for calculation of the total fracture energy for each case. When assigning the fracture energy in Abaqus for the small-scale yielding simulations, it is necessary to cut the energy in half since only a half model is used. Therefore, the calculated fracture energies, $\Gamma_{\mathrm{o}}$, were $6.075,7.0875,7.29$, and 7.59375 $\mathrm{MPa} \cdot \mathrm{mm}\left(\mathrm{kJ} / \mathrm{m}^{2}\right)$ for TH1-4, respectively.

Bilinear TS laws are used for the small-scale yielding simulations described in Chapter 4 for reasons discussed in Section 3.1.3. The peak traction and fracture energy were set equal to those defined by Tvergaard and Hutchinson so the change in shape caused a necessary modification to the critical separation, $\delta_{\mathrm{c}}$. Values for $\delta_{\mathrm{c}}$ can therefore be determined by substituting the calculated values of $\Gamma_{\mathrm{o}}$ and $\mathrm{T}_{\mathrm{o}}$ into Equation 2.6. For all TH materials, $\delta_{\mathrm{c}}=0.00675 \mathrm{~mm}$. This decision was made to minimize the effect of the substituted TS law shape on the macroscopic response of the system. Therefore, the final cohesive parameters for cases 1-4 can be seen in Table 3.5.

Based on the cohesive parameters defined in Table 3.5, it is expected that the bilinear tractionseparation response of the cohesive element would have data points as defined by Table 3.6. Figure 3.7 shows the desired bilinear responses for cases $1-4$ overlaid by the measured stress (traction) vs. applied displacement (separation) from the finite element analysis of the unit cell. It can be observed that the measured response of the cohesive elements matches exactly with the 
desired response for all four TH materials. As expected, all four of the TH traction-separation laws have a measured critical separation, $\delta_{\mathrm{c}}$, of $0.00675 \mathrm{~mm}$. The measured data from Figure 3.7 is compiled in Table 3.7.

\section{C2 Materials}

A similar unit cell analysis was performed for three sets of cohesive parameters to be used in future simulations with the $\mathrm{C} 2$ material. These cohesive materials are referred to as $\mathrm{C} 2$ _1, C2_2, and $\mathrm{C} 2$ 3. Cohesive parameters for these $\mathrm{C} 2$ materials can be found in

Table 3.8. These parameters were selected by comparing the C2 material's DWTT simulations with experimental data provided by CANMET.

The key points of the desired TS laws for the $\mathrm{C} 2$ materials can be found in

Table 3.9. The traction-separation response of these unit cell simulations can be found in Table 3.10 and Figure 3.8. The response of these cohesive elements in Abaqus perfectly matches the desired TS law for all three $\mathrm{C} 2$ materials.

As expected, $\mathrm{C} 2 \_2$ and $\mathrm{C} 2 \_3$ have a lower slope than $\mathrm{C} 2{ }_{-} 1$ in the elastic portion because they have reduced stiffness. The stiffnesses are reduced on these two materials to improve numerical stability in the SSY and DWTT simulations. The critical separation value, $\delta_{c}$, is $0.01488 \mathrm{~mm}$ for all three $\mathrm{C} 2$ materials. The thickness of the cohesive elements used for the $\mathrm{C} 2$ simulations is 0.03 $\mathrm{mm}$, rather than the $0.01 \mathrm{~mm}$ used for the TH materials, due to this much larger value of $\delta_{\mathrm{c}}$. The mesh deformation would be too great if a thinner element was used. 


\section{Conclusions}

It can be concluded that the parameters calculated to model the cohesive laws defined by Tvergaard and Hutchinson work as desired. The parameters chosen for the $\mathrm{C} 2$ materials also respond as desired.

The next step in the validation process is to re-create the small-scale yielding model developed by Tvergaard and Hutchinson (1992) to determine if that the bilinear laws outlined in this Chapter give equivalent results as the published trapezoidal laws when measuring the macroscopic response of the model. Resistance curves and CTOA data will be measured for each TS law for both the TH and C2 materials in Chapter 4. The CTOAs of the $\mathrm{C} 2$ and TH materials will be compared to each other.

Following this, the TS laws defined here will be used to simulate drop-weight tear tests in Chapter 5. Load vs. load-line displacement curves, crack extension vs. load-line displacement curves, and CTOA vs. crack extension curves will be measured for each of the seven materials. CTOAs measured during the DWTT simulations will be compared to those measured in the SSY simulations, and the CTOAs of the $\mathrm{C} 2$ materials will be compared to experimental observations. 


\begin{tabular}{|c|c|c|c|}
\hline Stress (MPa) & Plastic Strain & Stress (MPa) & Plastic Strain \\
\hline 600 & 0 & 910 & 0.190206919 \\
\hline 610 & 0.000539216 & 920 & 0.212519725 \\
\hline 620 & 0.001164143 & 930 & 0.237125475 \\
\hline 630 & 0.001886684 & 940 & 0.264231277 \\
\hline 640 & 0.002720164 & 950 & 0.294060429 \\
\hline 650 & 0.003679475 & 960 & 0.326853488 \\
\hline 660 & 0.004781227 & 970 & 0.362869405 \\
\hline 670 & 0.006043921 & 980 & 0.402386711 \\
\hline 680 & 0.007488126 & 990 & 0.445704781 \\
\hline 690 & 0.009136673 & 1000 & 0.493145151 \\
\hline 700 & 0.011014872 & 1010 & 0.545052911 \\
\hline 710 & 0.013150733 & 1020 & 0.60179817 \\
\hline 720 & 0.015575209 & 1030 & 0.663777594 \\
\hline 730 & 0.018322462 & 1040 & 0.731416024 \\
\hline 740 & 0.02143014 & 1050 & 0.80516817 \\
\hline 750 & 0.024939677 & 1060 & 0.8855204 \\
\hline 760 & 0.028896621 & 1070 & 0.972992606 \\
\hline 770 & 0.03335097 & 1080 & 1.068140168 \\
\hline 780 & 0.038357548 & 1090 & 1.171556007 \\
\hline 790 & 0.043976393 & 1100 & 1.283872744 \\
\hline 800 & 0.05027318 & 1110 & 1.405764953 \\
\hline 810 & 0.057319668 & 1120 & 1.537951527 \\
\hline 820 & 0.065194174 & 1130 & 1.681198149 \\
\hline 830 & 0.073982086 & 1140 & 1.836319877 \\
\hline 840 & 0.083776396 & 1150 & 2.004183852 \\
\hline 850 & 0.094678281 & 1160 & 2.185712122 \\
\hline 860 & 0.106797705 & 1170 & 2.381884595 \\
\hline 870 & 0.120254072 & 1180 & 2.593742129 \\
\hline 880 & 0.135176909 & 1190 & 2.822389747 \\
\hline 890 & 0.151706591 & 1200 & 3.069 \\
\hline 900 & 0.169995117 & & \\
\hline
\end{tabular}

Table 3.1: TH Material Plastic Strain 


\begin{tabular}{|cccccccccc|}
\hline Steel & $\mathrm{C}$ & $\mathrm{Mn}$ & $\mathrm{Si}$ & $\mathrm{Al}$ & $\mathrm{Nb}$ & $\mathrm{Ti}$ & $\mathrm{Cu}$ & $\mathrm{Cr}$ & $\mathrm{Ni}$ \\
\hline $\mathrm{C} 2$ & 0.04 & 1.56 & 0.24 & 0.039 & 0.069 & 0.013 & 0.31 & 0.07 & 0.11 \\
\hline
\end{tabular}

\begin{tabular}{|cccccccccc|}
\hline Steel & $\mathrm{P}$ & $\mathrm{S}$ & $\mathrm{Mo}$ & $\mathrm{Ca}$ & $\mathrm{Sn}$ & $\mathrm{B}$ & $\mathrm{N}$ & $\mathrm{Ce}$ & $\mathrm{V}$ \\
\hline $\mathrm{C} 2$ & 0.010 & $<0.009$ & 0.20 & 0.0021 & 0.014 & 0.0003 & 0.008 & 0.001 & 0.003 \\
\hline
\end{tabular}

Table 3.2: C2 Steel's Chemical Composition

\begin{tabular}{|cccccccc|}
\hline Steel & $\sigma_{\mathrm{y}}$ & UTS & Elongation & Area & $\mathrm{N}$ & $\mathrm{Y} / \mathrm{T}$ & $\mathrm{CVN}$ \\
& $(\mathrm{MPa})$ & $(\mathrm{MPa})$ & $(\%)$ & $\begin{array}{c}\text { Reduction } \\
(\%)\end{array}$ & & & $(\mathrm{J})$ \\
& & & & $(\%)$ & & \\
\hline $\mathrm{C} 2$ & 576 & 650 & 29.5 & 78.1 & 0.117 & 0.89 & 303 \\
\hline
\end{tabular}

Table 3.3: C2 Steel's Average Transverse Tensile and Charpy Properties (3 samples) 


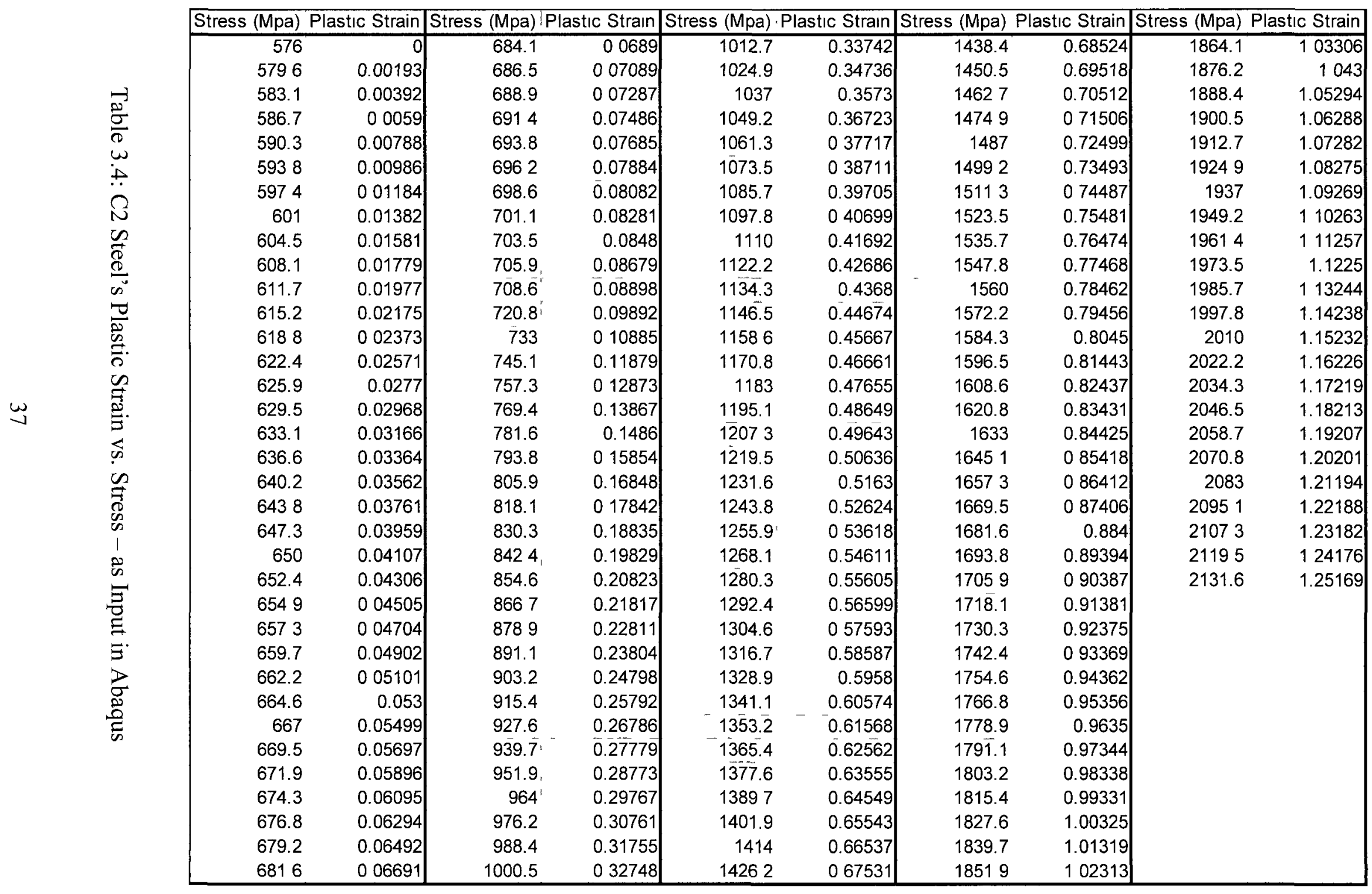




\begin{tabular}{|c|ccc|c|ccc|}
\hline \multirow{2}{*}{ Material } & \multicolumn{3}{|c|}{ MaxS Damage (MPa) } & Fracture Toughness & \multicolumn{3}{|c|}{ Elastic Moduli (MPa) } \\
\cline { 2 - 8 } & Normal & 1st & 2nd & Linear & Knn & Kss & Ktt \\
\hline TH1 & 1800 & 1350 & 1350 & 6.075 & 10000000 & 3846150 & 3846150 \\
TH2 & 2100 & 1575 & 1575 & 7.0875 & 10000000 & 3846150 & 3846150 \\
TH3 & 2160 & 1620 & 1620 & 7.29 & 10000000 & 3846150 & 3846150 \\
TH4 & 2250 & 1687.5 & 1687.5 & 7.59375 & 10000000 & 3846150 & 3846150 \\
\hline
\end{tabular}

Table 3.5: Cohesive Parameters - TH Materials

\begin{tabular}{|c|c|c|c|}
\hline \multicolumn{2}{|c|}{ TH1 } & \multicolumn{2}{c|}{ TH2 } \\
\hline Traction (MPa) & Separation (mm) & Traction (MPa) & Separation (mm) \\
\hline 0 & 0 & 0 & 0 \\
\hline 1800 & 0.00018 & 2100 & 0.00021 \\
\hline 0 & 0.00675 & 0 & 0.00675 \\
\hline
\end{tabular}

\begin{tabular}{|c|c|c|c|}
\hline \multicolumn{2}{|c|}{ TH3 } & \multicolumn{2}{c|}{ TH4 } \\
\hline Traction (MPa) & Separation (mm) & Traction (MPa) & Separation (mm) \\
\hline 0 & 0 & 0 & 0 \\
\hline 2160 & 0.00022 & 2250 & 0.00023 \\
\hline 0 & 0.00675 & 0 & 0.00675 \\
\hline
\end{tabular}

Table 3.6: Desired TS Laws for TH Materials, Cases 1-4 


\begin{tabular}{|c|c|c|c|c|c|c|c|c|}
\hline \multicolumn{9}{|c|}{ Materıal } \\
\hline \multicolumn{2}{|c|}{$\mathrm{TH} 1$} & \multicolumn{2}{|c|}{$\mathrm{TH} 2$} & \multicolumn{3}{|c|}{ TH3 } & \multicolumn{2}{|c|}{ TH4 } \\
\hline Traction (MPa) & Separation (mm) & Traction (MPa) & Separation $(\mathrm{mm})$ & Traction (MPa) & & Separation $(\mathrm{mm})$ & Traction (MPa) & Separation $(\mathrm{mm})$ \\
\hline 0.0 & 0.00000 & 0.0 & 0.00000 & 0.0 & & 0.00000 & 0.0 & 0.00000 \\
\hline 700.0 & 0.00007 & 700.0 & 0.00007 & 700.0 & 1 & 0.00007 & 700.0 & 0.00007 \\
\hline 1399.5 & 0.00014 & 1399.5 & 0.00014 & 1399.5 & & 0.00014 & 1399.5 & 0.00014 \\
\hline 1791.8 & 0.00021 & 2099.0 & 0.00021 & 2099.0 & & 0.00021 & 2099.0 & 0.00021 \\
\hline 1772.6 & 0.00028 & 2077.6 & 0.00028 & 2138.9 & · & 0.00028 & 2231.1 & 0.00028 \\
\hline 1753.5 & 0.00035 & 2055.1 & 0.00035 & 2115.7 & : & 0.00035 & 2206.9 & 0.00035 \\
\hline 1657.6 & 0.00070 & 1942.7 & 0.00070 & 2000.0 & & 0.00070 & 2086.2 & 0.00070 \\
\hline 1561.7 & 0.00105 & 1830.3 & 0.00105 & 1884.3 & ' & 0.00105 & 1965.6 & 0.00105 \\
\hline 1465.8 & 0.00140 & 1717.9 & 0.00140 & 1768.6 & ? & 0.00140 & 1844.9 & 0.00140 \\
\hline 1369.9 & 0.00175 & 1605.5 & 0.00175 & 1652.9 & 1 & 0.00175 & 1724.2 & 0.00175 \\
\hline 1274.0 & 0.00210 & 1493.1 & 0.00210 & 1537.2 & & 0.00210 & 1603.5 & 0.00210 \\
\hline 1178.1 & 0.00245 & 1380.8 & 0.00245 & 1421.5 & 1 & 0.00245 & 1482.8 & 0.00245 \\
\hline 1082.2 & 0.00280 & 1268.4 & 0.00280 & 1305.8 & ! & 0.00280 & 1362.1 & 0.00280 \\
\hline 986.3 & 0.00315 & 1156.0 & 0.00315 & 1190.1 & & 0.00315 & 1241.4 & 0.00315 \\
\hline 890.4 & 0.00350 & 1043.6 & 0.00350 & 1074.4 & & 0.00350 & 1120.7 & 0.00350 \\
\hline 794.6 & 0.00385 & 931.2 & 0.00385 & 958.7 & & 0.00385 & 1000.0 & 0.00385 \\
\hline 698.7 & 0.00420 & 818.8 & 0.00420 & 843.0 & & 0.00420 & 879.4 & 0.00420 \\
\hline 602.8 & 0.00455 & 706.5 & 0.00455 & 727.3 & I & 0.00455 & 758.7 & 0.00455 \\
\hline 506.9 & 0.00490 & 594.1 & 0.00490 & 611.6 & I & 0.00490 & 638.0 & 0.00490 \\
\hline 411.0 & 0.00525 & 481.7 & 0.00525 & 495.9 & & 0.00525 & 517.3 & 0.00525 \\
\hline 315.1 & 0.00560 & 369.3 & 0.00560 & 380.2 & I & 0.00560 & 396.6 & 0.00560 \\
\hline 219.2 & 0.00595 & 256.9 & 0.00595 & 264.5 & & 0.00595 & 275.9 & 0.00595 \\
\hline 123.3 & 0.00630 & 144.5 & 0.00630 & 148.8 & 1 & 0.00630 & 155.2 & 0.00630 \\
\hline 27.4 & 0.00665 & 32.1 & 0.00665 & 33.1 & & 0.00665 & 34.5 & 0.00665 \\
\hline 0.0 & 0.00675 & 0.0 & 0.00675 & 0.0 & & 0.00675 & 0.0 & 0.00675 \\
\hline
\end{tabular}




\begin{tabular}{|c|ccc|c|ccc|}
\hline \multirow{2}{*}{ Material } & \multicolumn{3}{|c|}{ MaxS Damage (MPa) } & $\begin{array}{c}\text { Fracture Toughness } \\
\text { (MPa-mm) }\end{array}$ & \multicolumn{3}{|c|}{ Elastic Moduli (MPa) } \\
\cline { 2 - 8 } & Normal & 1st & 2nd & Linear & Knn & Kss & Ktt \\
\hline C2_1 & 2016 & 1512 & 1512 & 15.00 & 10000000 & 3846154 & 3846154 \\
C2_2 & 2160 & 1620 & 1620 & 16.07 & 5000000 & 1923077 & 1923077 \\
C2_3 & 2304 & 1728 & 1728 & 17.14 & 5000000 & 1923077 & 1923077 \\
\hline
\end{tabular}

Table 3.8: Cohesive Parameters - C2 Materials

\begin{tabular}{|c|c|c|c|}
\hline \multicolumn{2}{|c|}{ C2_1 } & \multicolumn{2}{c|}{ C2_2 } \\
\hline Traction (MPa) & Separation (mm) & Traction (MPa) & Separation (mm) \\
\hline 0 & 0 & 0 & 0 \\
\hline 2016 & 0.0002016 & 2160 & 0.000432 \\
\hline 0 & 0.01488 & 0 & 0.01488 \\
\hline
\end{tabular}

\begin{tabular}{|c|c|}
\hline \multicolumn{2}{|c|}{ C2_3 } \\
\hline Traction (MPa) & Separation (mm) \\
\hline 0 & 0 \\
\hline 2304 & 0.0004608 \\
\hline 0 & 0.01488 \\
\hline
\end{tabular}

Table 3.9: Desired TS Laws for C2 Materials, Cases 1-3 


\begin{tabular}{|cc|cc|cc|}
\hline \multicolumn{3}{|c|}{ C2_1 } & \multicolumn{2}{c|}{ C2_2 } & \multicolumn{2}{c|}{ C2_3 } \\
\hline Traction (MPa) & Separation (mm) & Traction (MPa) Separation (mm) & Traction (MPa) Separation (mm) \\
\hline 0.0 & 0.0000 & 0.0 & 0.0000 & 0.0 & 0.0000 \\
1499.9 & 0.0001 & 750.0 & 0.0002 & 750.0 & 0.0002 \\
2002.5 & 0.0003 & 1500.1 & 0.0003 & 1500.1 & 0.0003 \\
1981.9 & 0.0004 & 2157.3 & 0.0005 & 2250.2 & 0.0005 \\
1961.3 & 0.0006 & 2134.9 & 0.0006 & 2281.8 & 0.0006 \\
1837.7 & 0.0015 & 2000.3 & 0.0015 & 2137.9 & 0.0015 \\
1755.3 & 0.0021 & 1910.6 & 0.0021 & 2042.0 & 0.0021 \\
1631.7 & 0.0030 & 1776.1 & 0.0030 & 1898.2 & 0.0030 \\
1487.5 & 0.0041 & 1619.1 & 0.0041 & 1730.4 & 0.0040 \\
1343.3 & 0.0051 & 1462.1 & 0.0051 & 1562.6 & 0.0051 \\
1219.7 & 0.0060 & 1327.5 & 0.0060 & 1418.8 & 0.0060 \\
1075.5 & 0.0070 & 1170.6 & 0.0071 & 1251.0 & 0.0071 \\
931.3 & 0.0081 & 1013.6 & 0.0081 & 1083.3 & 0.0081 \\
807.7 & 0.0090 & 879.1 & 0.0090 & 939.4 & 0.0090 \\
663.5 & 0.0100 & -122.1 & 0.0100 & 771.6 & 0.0100 \\
519.3 & 0.0111 & 565.1 & 0.0111 & 603.8 & 0.0111 \\
395.7 & 0.0120 & 430.6 & 0.0120 & 460.0 & 0.0120 \\
251.5 & 0.0131 & 273.6 & 0.0130 & 292.2 & 0.0130 \\
107.3 & 0.0141 & 116.6 & 0.0141 & 124.4 & 0.0141 \\
4.2 & 0.01485 & 4.5 & 0.01485 & 4.6 & 0.01485 \\
0.0 & 0.01488 & 0.0 & 0.01488 & 0.0 & 0.01488 \\
\hline
\end{tabular}

Table 3.10: Measured Results From Unit Cell Analyses for C2 Materials 


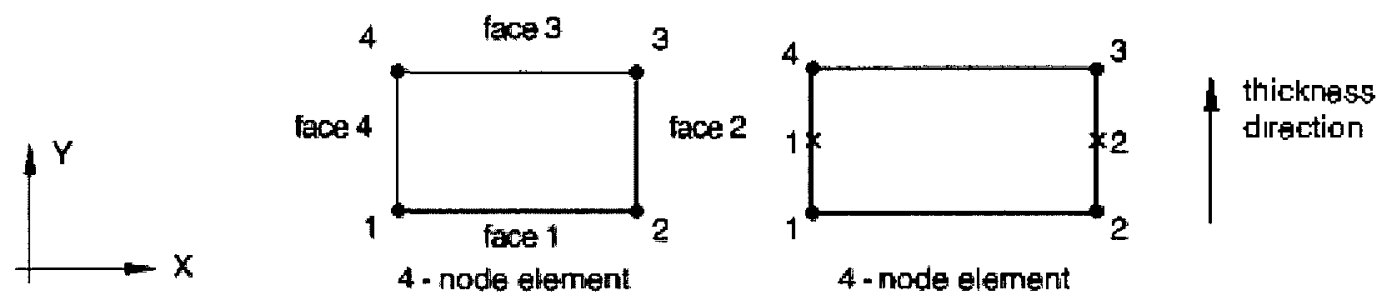

Figure $3.1: \mathrm{COH} 2 \mathrm{D} 4$ Element Numbering Conventions $(\bullet-$ Nodes, $\times$ - Integration points) (Abaqus, 2008)

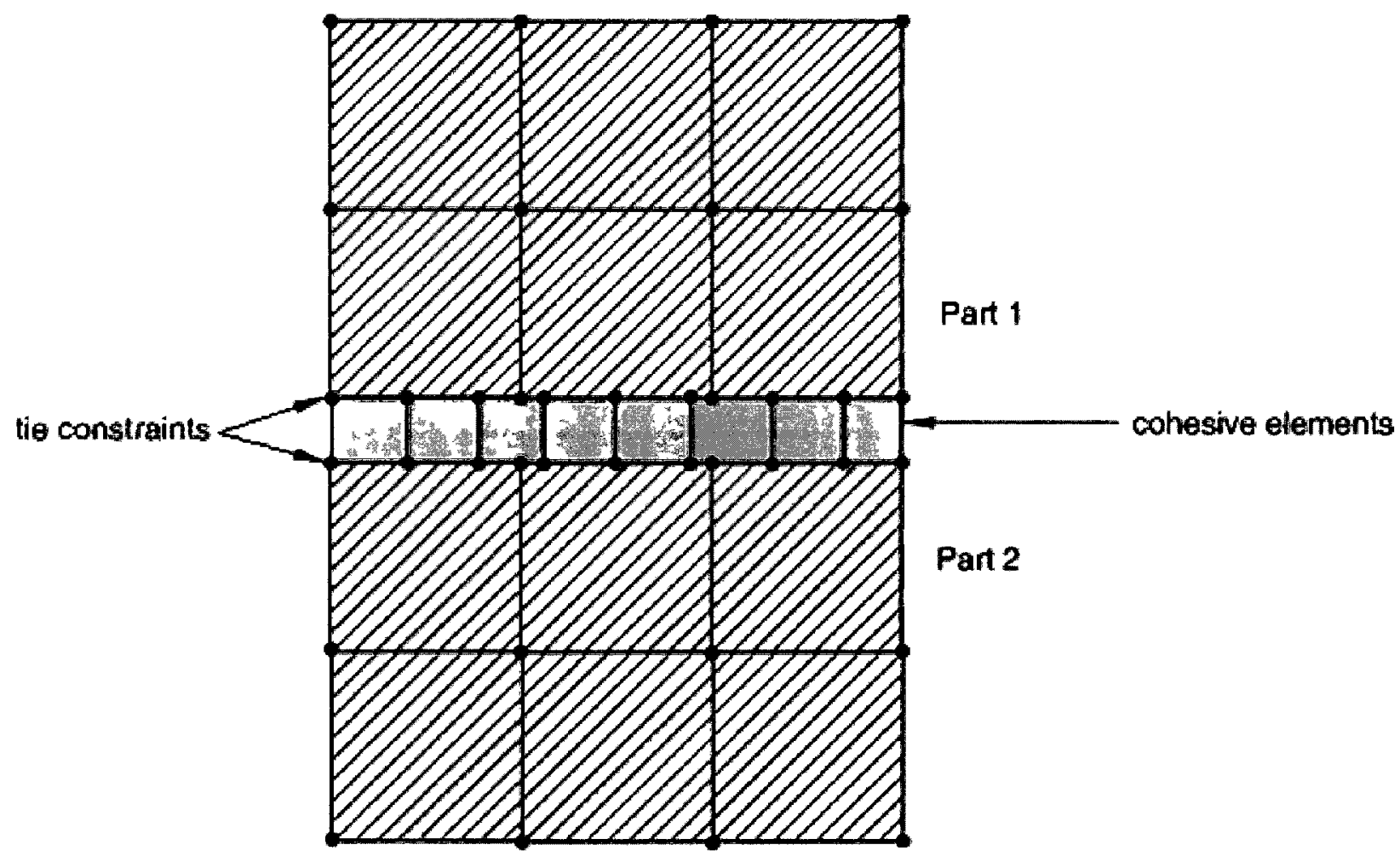

Figure 3.2: Typical Traction-Separation Based Cohesive Zone Model - Independent Meshes with Tie Constraints (Abaqus, 2008) 


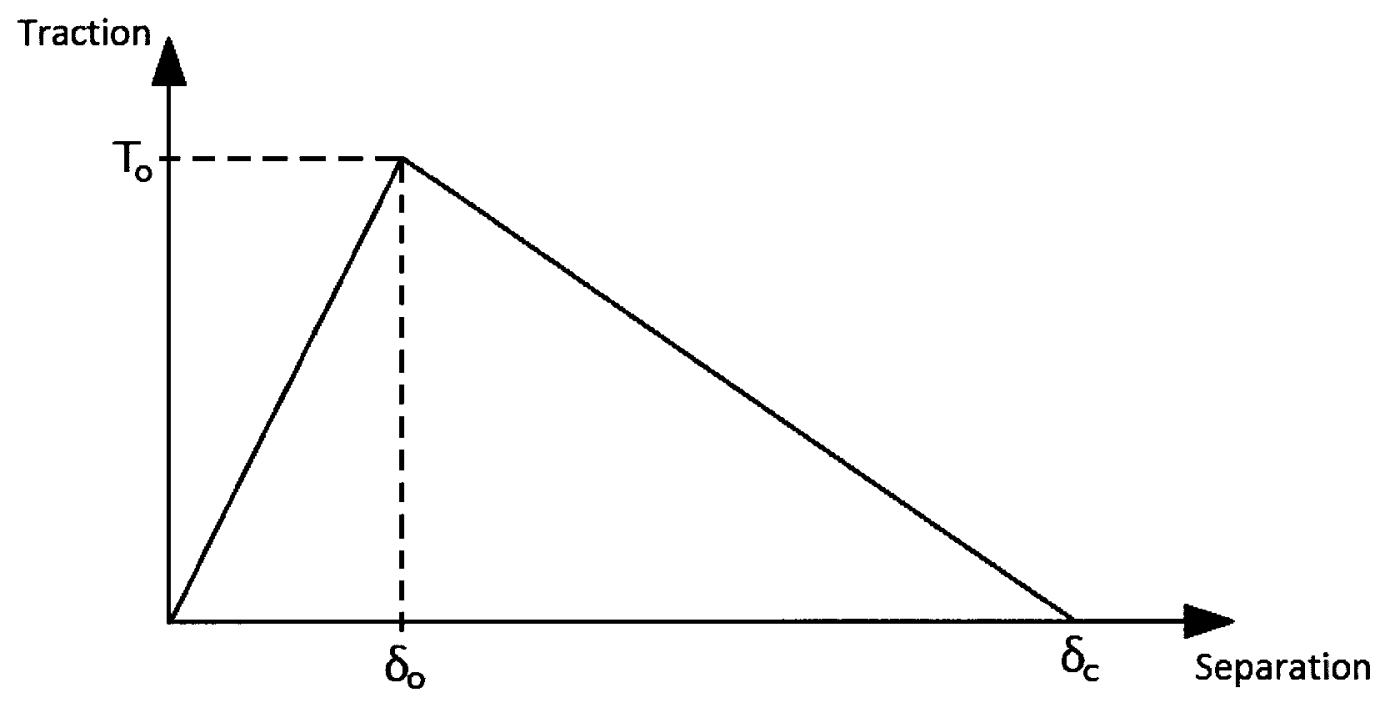

Figure 3.3: Typical Traction-Separation Response

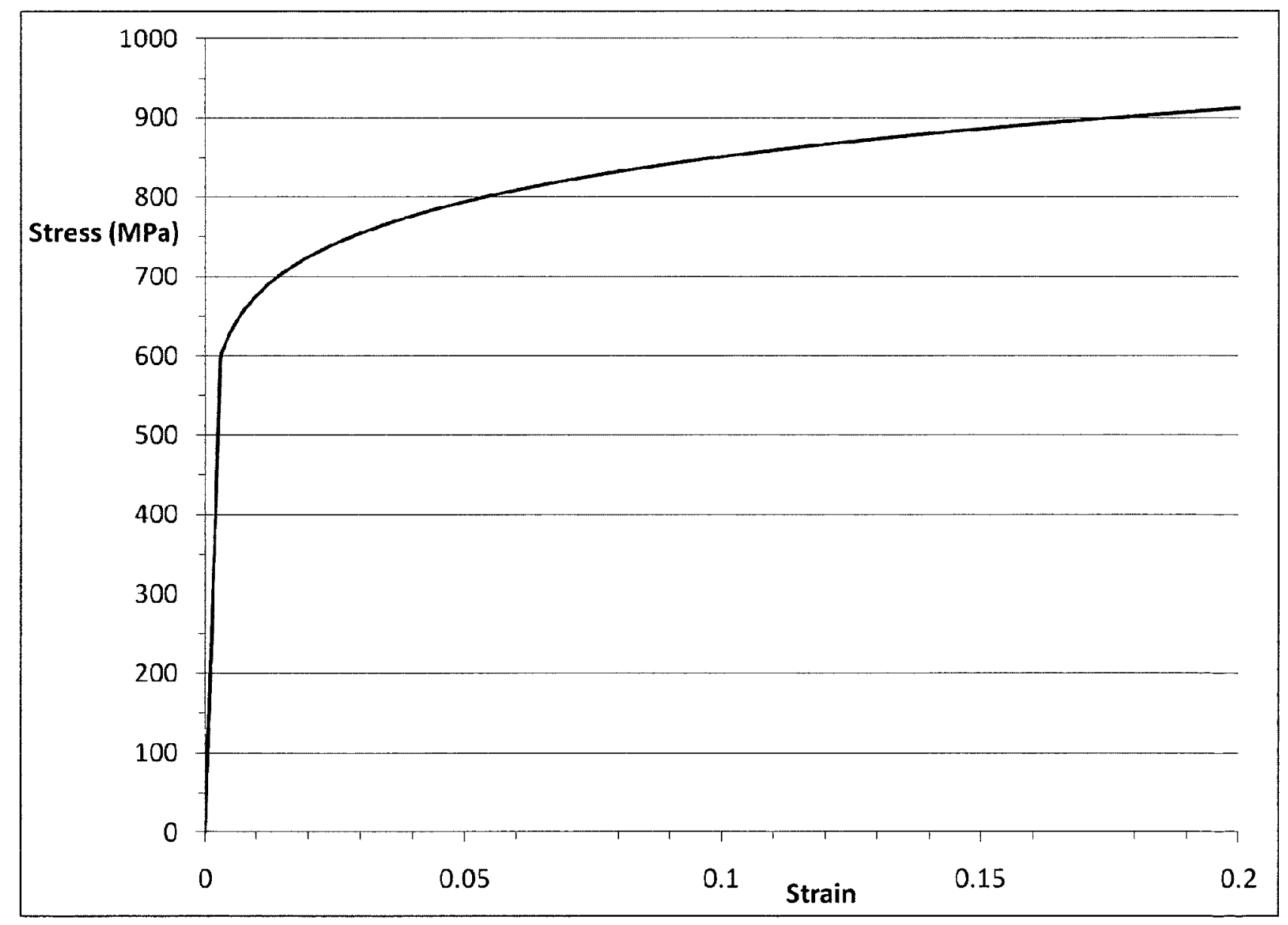

Figure 3.4: TH Steel - True Stress-Strain Curve 


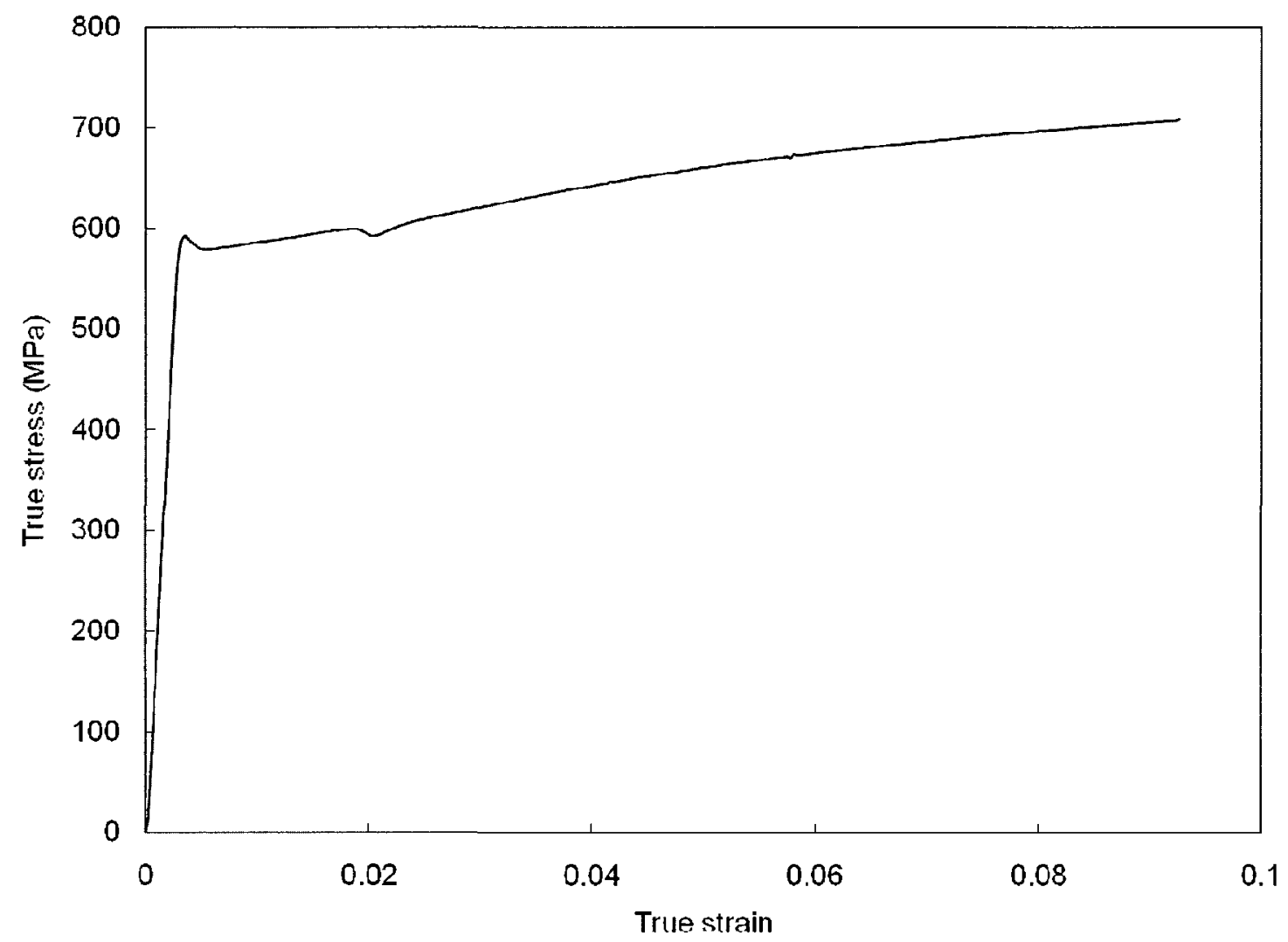

Figure 3.5: C2 Steel - True Stress-Strain Curve (Su, 2010a)

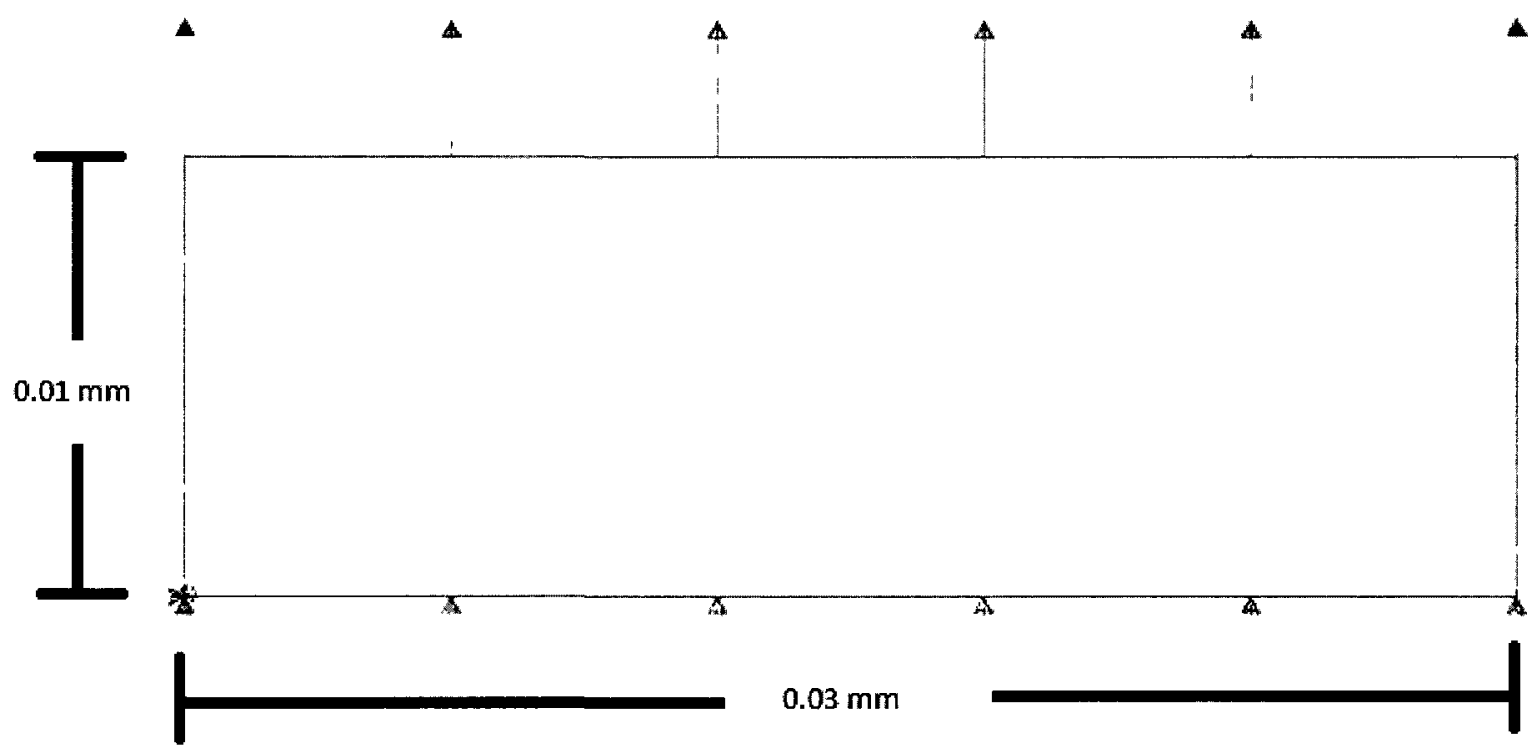

Figure 3.6: Unit Cell Geometry and Boundary Conditions 


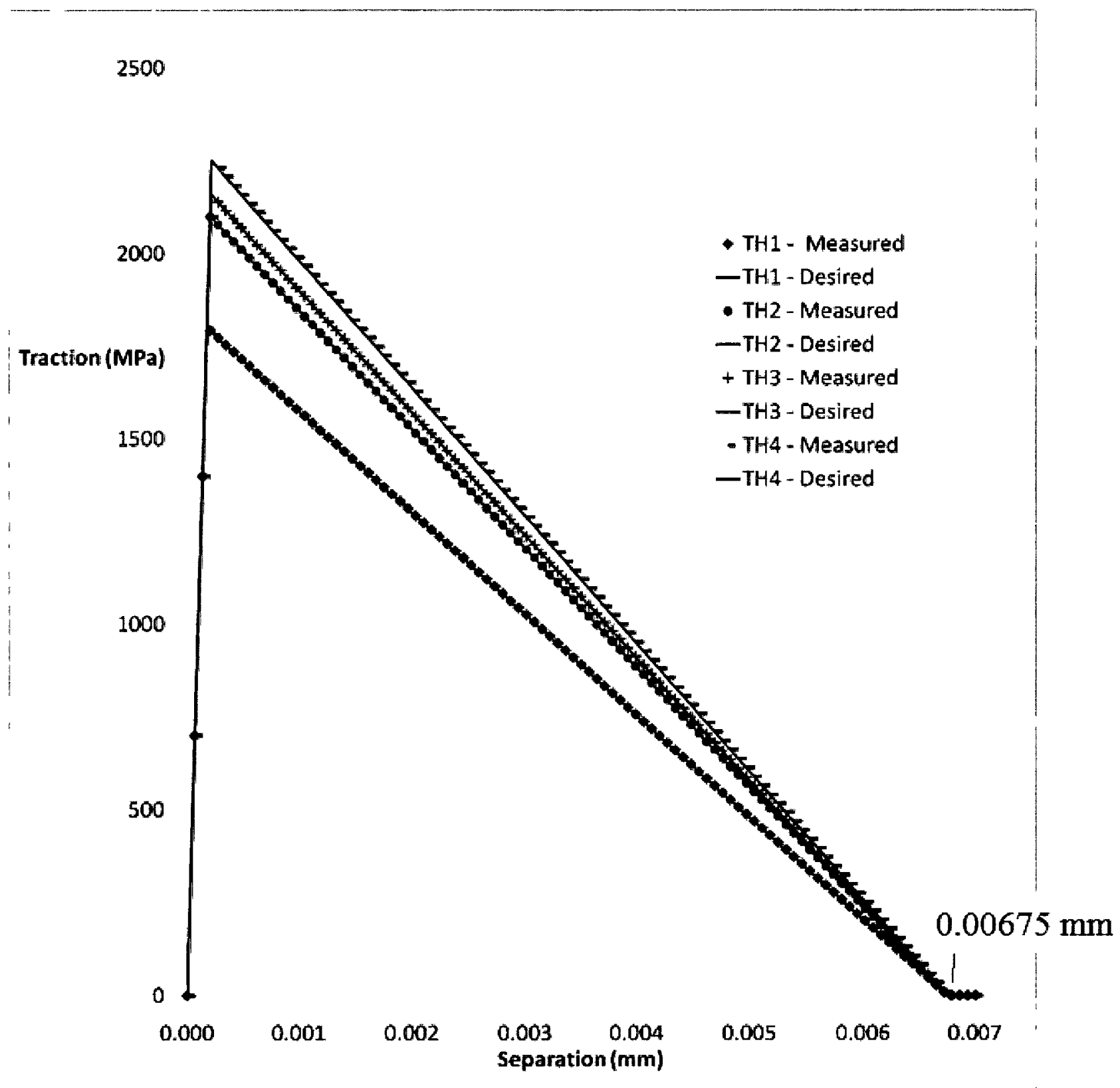

Figure 3.7: Unit Cell Analysis, Verification of TS Laws for TH Materials 


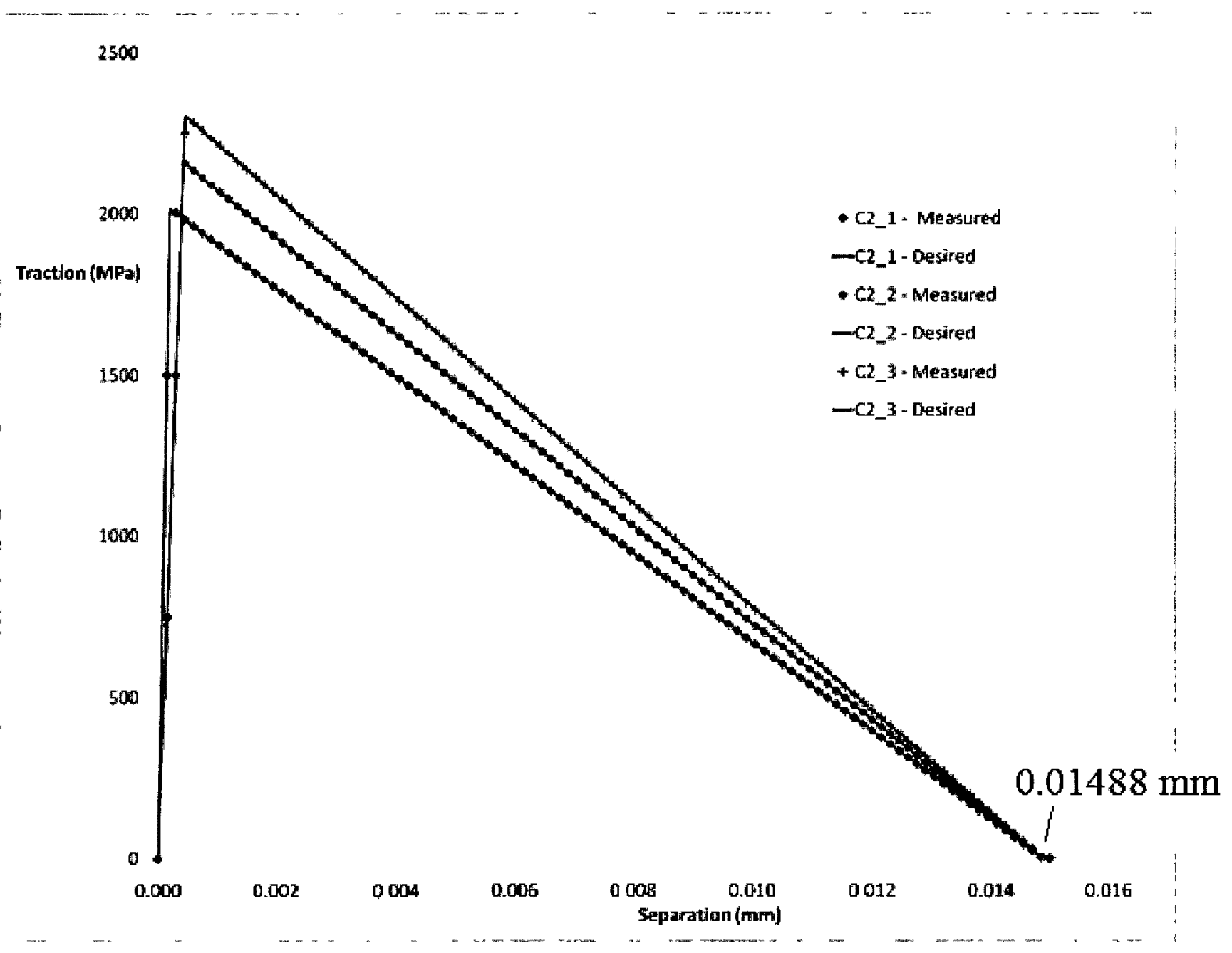

Figure 3.8: Unit Cell Analysis, Verification of TS Laws for C2 Materials 


\section{Chapter 4}

\section{FE Simulation of Small-Scale Yielding Models}

This chapter will explore implementation of cohesive zone (CZ) modeling in Abaqus by performing finite element analysis of a small-scale yielding (SSY) model. The SSY model will be defined and simulation results for a set of ideal materials, as defined by Tvergaard and Hutchinson (1992), and a set of real-world materials (C2 steel) will be presented. Tvergaard and Hutchinson used a CZ model to perform analysis of plane strain mode I crack growth under conditions of small-scale yielding. A small-scale yielding analysis is performed here to verify that the techniques used here are able to recreate the results of Tvergaard and Hutchinson (1992). Verifying the techniques used here by comparing with Tvergaard and Hutchinson's results will increase the confidence in the DWTT results detailed in Chapter 5. In addition, the crack propagation in C2 steels is simulated using the SSY model. Simulation results for both the TH and $\mathrm{C} 2$ materials include normalized resistance curves and crack tip opening angle data.

\subsection{Model Specifications}

\section{Geometry}

A small scale yielding model is used to simulate a pre-existing horizontal crack surrounded by an infinite plate. Figure 4.1 shows the geometry for the small-scale yielding model. The initial crack extends along the bottom edge from the left side of the semicircle to the center. Only the top side of the crack needs to be modeled due to the inherent symmetry about the crack front for mode-I crack growth. The crack is modeled by leaving the current crack face unconstrained and constraining the material to the right of center in the y-direction, along the line of symmetry. $A_{o}$ is the radius of the circle, and the initial length of the crack. A value of $200 \mathrm{~mm}$ was chosen for $A_{0} . B_{0}$ is the equal to twice the length of the cohesive zone, which in this case was $9 \mathrm{~mm}$. This allows for up to $4.5 \mathrm{~mm}$ of crack growth. $\Delta_{\mathrm{o}}$ is the length of the smallest elastic-plastic elements at the crack tip, and is set to $0.1 \mathrm{~mm}$. This means that $1 / 2 \mathrm{~B}_{0}=45 \Delta_{0}$ and $A_{0}=2000 \Delta_{0}$. These values were selected to meet small-scale yielding requirements which require the plastic zone to 
be less than $5 \%$ of the radius of the model. The largest plastic zone simulated here is $2.354 \mathrm{~mm}$, which is only about $1.2 \%$ of the radius.

\section{Material Properties}

Two different sets of materials were used in this model, with each set having one elastic-plastic response, and varying cohesive parameters. The first set of four materials was defined by Tvergaard and Hutchinson (1992) and is representative of an ideal high strength steel. These are the TH materials defined in Chapter 3. The second material set is the $\mathrm{C} 2$ steel. The $\mathrm{C} 2$ steel is an X70 steel characterized by CANMET/MTL (Xu, 2010a) and is representative of modern high toughness pipeline steels. The $\mathrm{C} 2$ material is also defined in Chapter 3. Cohesive parameters for the TH materials can be found in Table 3.5. Cohesive parameters for the $\mathrm{C} 2$ materials can be found in Table 3.8. As mentioned in Chapter 3, bilinear traction-separation laws were used for these simulations, rather than the trapezoidal laws used by Tvergaard and Hutchinson.

\section{Loading Conditions}

It is possible to apply a ramped displacement field to the outer edges of the model to generate a known stress intensity factor at the crack tip. To do this, the semicircle is broken up into 36 wedges, each 5 degrees of the circle, as shown in Figure 4.2. Displacements $u_{x}(t)$ and $u_{y}(t)$ are then applied to the nodes at the outer edges based on the following equations (Williams, 1957):

$$
\begin{aligned}
& u_{x}(t)=\left\{\frac{K_{r}}{2 \mu} \sqrt{\frac{A_{o}}{2 \pi}} \cos \left(\frac{\theta}{2}\right)\left[\kappa-1+2 \sin ^{2}\left(\frac{\theta}{2}\right)\right]\right\} \cdot \frac{t}{t_{f}} \quad, \quad \mathrm{t}=0 \rightarrow \mathrm{t}_{f} \\
& u_{y}(t)=\left\{\frac{K_{r}}{2 \mu} \sqrt{\frac{A_{o}}{2 \pi}} \sin \left(\frac{\theta}{2}\right)\left[\kappa+1-2 \cos ^{2}\left(\frac{\theta}{2}\right)\right]\right\} \cdot \frac{t}{t_{f}} \quad, \quad \mathrm{t}=0 \rightarrow \mathrm{t}_{f}
\end{aligned}
$$

Where $K_{r}$ is the maximum desired stress intensity at the crack tip (set high enough to allow complete propagation of the crack though the cohesive zone), $\mu$ is the shear modulus and $\kappa=3-4 v$, where $v$ is Poisson's ratio. $\theta$ is measured counter-clockwise from the crack front. 
Time varies from 0 to 1 second, which causes the displacements to increase linearly throughout the simulation.

Table 4.1(a) details the maximum displacements applied for the TH materials. These displacements are based on a maximum applied stress intensity of $9135 \mathrm{MPa} \sqrt{\mathrm{mm}}$. This value is equal to $5 \cdot K_{o}^{T H 4}$, and was chosen to be sufficiently large to allow the crack to propagate completely through the cohesive layer. $K_{o}^{T H 4}$ is the $K_{o}$ for the TH4 material based on Equation (2.7) and represents the largest $K_{o}$ among the TH materials.

Table 4.1(b) details the maximum displacements applied for the $\mathrm{C} 2$ materials. These displacements are based on a maximum applied stress intensity of 41,952 $\mathrm{MPa} \sqrt{\mathrm{mm}}$. This value is $\sim 15.5 \cdot K_{o}^{C 2}{ }^{3}$, and was large enough to allow full propagation of the crack for the $\mathrm{C} 2$ simulations. $K_{o}^{C 2 \_3}$ is the $K_{o}$ for the $\mathrm{C}_{-} \_3$ material based on Equation (2.7) and represents the largest $K_{o}$ among the $\mathrm{C} 2$ materials.

\section{Mesh Design}

The model is divided into two separate parts for meshing. There is the main semicircular section which is divided into 1,533 solid quadrilateral plane-strain elements with reduced integration (CPE4R elements), and a cohesive layer along the crack front composed of 150 cohesive (COH2D4) elements. The two sections are joined together using surface-to-surface tie constraints. Figure 4.3 and Figure 4.4 (a) - (c) show the mesh used for these simulations at increasing levels of magnification of the crack tip. To mesh the elastic-plastic region, a swept meshing technique is used away from the crack tip and a structured meshing technique is used at the crack tip. A free meshing technique joins the gap between these two sections. The cohesive layer is meshed using a swept meshing technique, as required by the element type. For the TH material the cohesive elements have a length of $0.03 \mathrm{~mm}$, and a thickness of $0.01 \mathrm{~mm}$. This is too thin for the tougher $\mathrm{C} 2$ materials though, as the required maximum separation value of 0.01488 
$\mathrm{mm}$ would cause too much mesh distortion. Therefore, cohesive elements with a length of 0.03 $\mathrm{mm}$ and a thickness of $0.03 \mathrm{~mm}$ were used when modeling the $\mathrm{C} 2$ materials.

The smallest elastic-plastic elements, those that are tied to the cohesive elements, have dimensions of $0.1 \times 0.1 \mathrm{~mm}$. Diehl (2007) recommends that 3-5 cohesive elements be used per adjacent continuum element. The dimensions used here means that there are $31 / 3$ cohesive elements per adjacent continuum element. It was found to be particularly important that the cohesive nodes do not line up with the elastic-plastic nodes too often, as it would allow the mesh to deform in an undesirable fashion.

\section{Verification of Applied Stress Intensity}

A simulation was performed without the cohesive layer to verify that the applied displacement field is yielding the desired stress intensity at the crack tip. Using the TH materials, a displacement field was applied, based on Equations 4.1 and 4.2, until a stress intensity higher than the highest critical stress intensity was reached (that of the TH4 material). The assumed stress intensity at the crack tip was compared with the measured J-integral for 8 contours in Abaqus. The J-integral data can be found in Table 4.2. Table 4.2 also provides incremental percentage difference information between the theoretical and measured intensities. It is seen that the two sets of data agree almost perfectly, with errors of less than $0.5 \%$ throughout the simulation.

\subsection{Results}

Simulations were completed in Abaqus/Explicit, version 6.8-2 (Abaqus, 2008), using the CAE platform on Windows 7 computers. Abaqus/Explicit was chosen rather than Abaqus/Standard because it is more numerically stable for the simulations performed here. To simulate quasi-static conditions, dynamic, explicit steps were used with a time period of one second. Results were recorded at 8000 increments, divided into approximately equal intervals based on time. Mass densities of $7.85 \times 10^{-6} \mathrm{~kg} / \mathrm{mm}^{3}$ were used for all materials. Typical run times for these simulations ranged from 3-5 minutes for the TH1 simulation to up to 120 minutes for the $\mathrm{C}_{-} 3$ 
simulation. The range of simulation time was due to the reduction of the stable time increment caused by increased fracture energy at the crack tip.

\subsubsection{Results of TH Simulations}

\section{Resistance Curves}

Four small-scale yielding simulations were run with the same mesh, loading conditions, and TH elastic-plastic material (Table 3.1). The TH material has a yield stress, $\sigma_{y}$, of $600 \mathrm{MPa}$, Young's Modulus, E, of 200,000 MPa, and Poisson's ratio, v, of 0.3. The applied displacement field for the TH simulations can be found in Table 4.1(a). Each case had increasingly tough cohesive parameters, as defined in Table 3.5. These sets of cohesive parameters are referred to as TH1TH4. The peak traction in the normal direction was defined as $3.0 \cdot \sigma_{y}, 3.5 \cdot \sigma_{y}, 3.6 \cdot \sigma_{y}$, and 3.75. $\sigma_{y}$ for TH1-TH4 respectively. These peak tractions can be found in Table 4.3 (a). Fracture energies, $\Gamma_{o}$, were calculated as if a trapezoidal TS law was used, as per Equation 2.5. As in the simulations performed by Tvergaard and Hutchinson (1992), $\delta_{c}=0.1 \Delta_{o}, \frac{\delta_{1}}{\delta_{c}}=0.15$, and $\frac{\delta_{2}}{\delta_{c}}=0.5$. These calculated fracture energies were then inserted into the bilinear TS laws in Abaqus, so the only the shape of the TS laws used here is different than the TS laws used by Tvergaard and Hutchinson (1992). The fracture energy input into Abaqus was defined as half the fracture energy calculated for the model as only a half-model was analyzed. Values quoted for fracture energies in this thesis are those entered as material parameters into Abaqus (equal to half the fracture energy of the whole model). These fracture energies can be found in Table 4.3 (a).

It was possible to measure the crack length from the simulation results by measuring the traction stresses in each element in the cohesive layer. The cohesive elements are numbered sequentially from 1-150, with element 1 at the original crack tip. The current crack tip is defined as the highest element number to have failed completely, with traction capability reduced to zero. Tables 4.4-4.7 show sample traction data from the simulations for TH1-4 respectively. The crack length, $\Delta \mathrm{a}$, can be determined by multiplying the length of the cohesive element by the element 
number of the current crack tip. The crack length is then normalized by the size of the plastic zone $\left(R_{0}\right)$ for each case. $R_{0}$ can be found by:

$$
R_{o}=\frac{1}{3 \pi\left(1-v^{2}\right)} \frac{E \Gamma_{o}}{\sigma_{y}^{2}}=\frac{1}{3 \pi}\left(\frac{K_{o}}{\sigma_{y}}\right)^{2}
$$

Values for the ratio of the peak traction to yield stress, $\hat{\sigma} / \sigma_{y}$, fracture energy, $\Gamma_{o}$, critical stress intensity, $K_{o}$, and plastic zone length, $R_{o}$, for each TH case can be found in Table 4.3(a).

The tractions are then compared to the applied displacement in the x-direction at the outer node at $0^{\circ}$. This displacement is converted into a theoretical applied stress intensity, $\mathrm{K}_{\mathrm{r}}$, using Equation (4.1), which is then normalized by the critical stress intensity, $K_{0}$, for each case. The normalized values $\mathrm{K}_{\mathrm{r}} / \mathrm{K}_{\mathrm{o}}$ can be found for each increment in Tables 4.4-4.7.

Figure 4.5 shows the results of these four simulations by plotting $K_{r} / K_{0}$ vs. $\Delta a / R_{0}$. As expected, increasing the toughness of the cohesive elements increases the fracture resistance. On the same graph, the results published by Tvergaard and Hutchison (1992) are displayed with dashed lines. It is seen that the steady-state resistance from these four simulations agrees quite closely with the Tvergaard and Hutchinson steady-state resistance data. Differences in the shape of the resistance curves before steady-state can likely be attributed to the difference in shape between the two sets of traction-separation laws.

\section{Crack Tip Opening Angle}

The crack tip opening angle was measured for each simulation at several increments. The ISO defines the CTOA as the angle of the crack surfaces measured (or calculated) at $1 \mathrm{~mm}$ from the current crack tip (ISO, 2007). The crack tip in the $\mathrm{CZ}$ simulations performed here is defined to be located at the shared nodes between the last cohesive element to fail (element fully damaged, 
with no ability to transfer loads), and the next cohesive element that will fail. The elastic-plastic nodes do not line up exactly $1 \mathrm{~mm}$ behind this crack tip due to the length difference between the cohesive and elastic-plastic elements, but to remain consistent with the spirit of the ISO approach, the CTOA was calculated by measuring the y-displacement of the node of an elasticplastic element that was nearest to $1 \mathrm{~mm}$ behind the current crack tip. This displacement was used as the crack tip opening displacement (CTOD). The CTOA was then calculated using from the CTOD using trigonometry and calculating with the true distance behind the crack tip. Figure 4.6 shows an example of how the CTOA is calculated by overlaying the original transparent mesh by a darkened mesh after the crack has extended about $3.2 \mathrm{~mm}$. The cohesive layer is hidden to allow the CTOA to be seen more clearly. It can be seen that the new surface created by the crack propagation is not completely flat. This will introduce some 'noise' into the CTOA data when using this calculation method, but the average measured CTOA at steady-state should provide a reasonable approximation of the true results. No data for CTOA is calculated until the crack length reaches $\sim 1 \mathrm{~mm}$.

Table 4.8 shows some sample displacement data for the TH1 case for nodes labelled by their distance from the original crack tip to demonstrate the process of calculating the CTOA. Tables 4.9-4.11 present the CTOA data calculated from the results of the simulations for TH2-TH4, respectively. The CTOA vs. crack length, $\Delta \mathrm{a}$, has been plotted for all four cases in Figure 4.7. Power law trendlines were fitted using MS Excel. As expected, increasing fracture toughness causes increasing critical CTOAs. Additionally, the numerical results follow the expected trends, as the CTOA values are initially high, but drop towards a steady-state value after a small amount of propagation. The length of the cohesive zone $(4.5 \mathrm{~mm})$ was not enough to conclusively achieve steady-state CTOAs for $\mathrm{TH} 2-4$, but it can be seen that a steady-state value of about $1.9^{\circ}$ was determined for the TH1 material. Values for steady-state CTOAs are estimated from their power law trendlines as about $2.5^{\circ}, 3.5^{\circ}$, and $6^{\circ}$ for $\mathrm{TH} 2-4$ respectively. These values will be compared to the steady-state CTOAs measured for these materials during DWTT simulations in Chapter 5. 


\subsubsection{Results of C2 Simulations}

\section{Resistance Curves}

Three small-scale yielding simulations were run with the same mesh, loading conditions, and C2 elastic-plastic material (Table 3.4). The $\mathrm{C} 2$ material has a yield stress, $\sigma_{y}$, of $576 \mathrm{MPa}$, Young's Modulus, E, of 195,400 MPa, and Poisson's ratio, $v$, of 0.3 . The applied displacement field for the C2 simulations can be found in Table 4.1(b). Each case had increasingly tough cohesive parameters, as defined in Table 3.8. These sets of cohesive parameters are referred to as C2_1C2_3. The peak traction in the normal direction was defined as $3.5 \cdot \sigma_{y}, 3.75 \cdot \sigma_{y}$, and $4.0 \cdot \sigma_{y}$ for C2_1-C2_3 respectively. Values for $\hat{\sigma} / \sigma_{y}, \Gamma_{o}, K_{o}$, and $R_{o}$ for each $\mathrm{TH}$ case can be found in Table 4.3(b). Fracture energies were calculated as per Equation 2.5 again, as if $\delta_{c}=0.1 \Delta_{o}$,

$\frac{\delta_{1}}{\delta_{c}}=0.15$, and $\frac{\delta_{2}}{\delta_{c}}=0.5$. This technique for choosing $\Gamma_{o}$, and therefore $\delta_{c}$, is used to remain consistent with the TH simulations instead of arbitrarily assigning values. Tables 4.12-4.14 show sample resistance curve data from the simulations for $\mathrm{C} 2 \_1-\mathrm{C} 2 \_3$ materials respectively.

Figure 4.8 shows the results of these three simulations by plotting $K_{r} / K_{o} v s . \Delta a / R_{0}$. Again as expected, increasing the toughness of the cohesive elements increases the fracture resistance. Data for the $\mathrm{C}_{2} 3$ simulation is slightly more sporadic as the high toughness of the cohesive elements meant there was a much larger amount of mesh deformation required for crack propagation. This can be seen in the CTOA data presented below. It is likely that a larger model with coarser mesh would be able to achieve more data points along this resistance curve, but as the same issues are not experienced for the DWTT simulations with the same parameters, it is not further explored here.

\section{Crack Tip Opening Angle}

CTOA data for the C2_1-C2_3 materials can be found in Table 4.15(a) - (c). The CTOAs are obtained following the same procedure used for the TH1-TH4 materials. This data is plotted in Figure 4.9. Again, the length of the cohesive zone $(4.5 \mathrm{~mm})$ was not enough to conclusively 
achieve steady-state CTOAs for these materials, and no estimates of the steady-state CTOA will be given here. Trendlines were fitted to the CTOA data using power laws in Excel. The trendline for the C2 3 data is extended forward 1 period to give a better idea of what the limited data is predicting. The CTOA values are $\sim 30^{\circ}-80^{\circ}$ at initiation and drop to $\sim 20^{\circ}-60^{\circ}$ after $2 \mathrm{~mm}$ of propagation for the $\mathrm{C} 2$ materials. As expected, due to the higher fracture energy of the $\mathrm{C} 2$ materials, the CTOAs for the $\mathrm{C} 2$ materials are much higher than those of the TH materials. These results will be compared to the CTOAs measured for these materials during DWTT simulations in Chapter 5.

\subsection{Conclusions}

Small-scale yielding simulations were carried out for two different sets of materials. Verification of the model design was performed by first measuring the theoretical applied vs. measured stress intensity at the crack tip. Following this, simulations performed by Tvergaard and Hutchinson (1992) were recreated and the results were compared. It was seen that there was very good correlation between the resistance curves found here, and those published by Tvergaard and Hutchinson. CTOA data was collected for the TH materials, and it was seen, as expected, that tougher cohesive elements resulted in higher values of the CTOA. Additionally, the CTOA values followed trends observed experimentally (Newman, 2003), as they started high, and steadily dropped towards steady-state values. Steady-state CTOA values ranged from $\sim 2-6^{\circ}$ for the TH materials. These values will be compared to the values found during the DWTT simulations in Chapter 5.

The tougher C2 materials were seen to yield even higher resistance curves, and higher values for the CTOAs. While steady-state values for CTOA were not achieved, measured values of CTOA ranged from $\sim 30^{\circ}-80^{\circ}$ at initiation, to $\sim 20^{\circ}-60^{\circ}$ after $2 \mathrm{~mm}$ of propagation for the $\mathrm{C} 2$ materials. These values will be compared to the values found during the DWTT simulations in Chapter 5 . 


\begin{tabular}{|c|c|c|}
\hline Angle & Ux & Uy \\
\hline 0 & 0.268 & 0 \\
5 & 0.26902 & 0.01175 \\
10 & 0.27205 & 0.0238 \\
15 & 0.27703 & 0.03647 \\
20 & 0.28383 & 0.05005 \\
25 & 0.29229 & 0.0648 \\
30 & 0.30222 & 0.08098 \\
35 & 0.31338 & 0.09881 \\
40 & 0.32549 & 0.11847 \\
45 & 0.33825 & 0.14011 \\
50 & 0.35135 & 0.16384 \\
55 & 0.36443 & 0.18971 \\
60 & 0.37715 & 0.21775 \\
65 & 0.38916 & 0.24792 \\
70 & 0.40009 & 0.28015 \\
75 & 0.40961 & 0.3143 \\
80 & 0.41736 & 0.35021 \\
85 & 0.42305 & 0.38766 \\
90 & 0.42639 & 0.42639 \\
95 & 0.42711 & 0.46611 \\
100 & 0.42499 & 0.50649 \\
105 & 0.41987 & 0.54718 \\
110 & 0.41159 & 0.58781 \\
115 & 0.40006 & 0.62797 \\
120 & 0.38525 & 0.66727 \\
125 & 0.36716 & 0.70531 \\
130 & 0.34584 & 0.74166 \\
135 & 0.32141 & 0.77595 \\
140 & 0.29401 & 0.80779 \\
145 & 0.26384 & 0.83681 \\
150 & 0.23116 & 0.86269 \\
155 & 0.19623 & 0.88512 \\
160 & 0.15937 & 0.90386 \\
165 & 0.12094 & 0.91866 \\
170 & 0.08131 & 0.92936 \\
175 & 0.04086 & 0.93584 \\
180 & 0 & 0.938 \\
\hline & & \\
\hline
\end{tabular}

(a) TH Materials $-K_{r}^{\max }=9135$

\begin{tabular}{|c|c|c|}
\hline Angle & Ux & Uy \\
\hline 0 & 1.23079 & 0 \\
5 & 1.23546 & 0.05394 \\
10 & 1.24939 & 0.10931 \\
15 & 1.27223 & 0.16749 \\
20 & 1.30346 & 0.22984 \\
25 & 1.34234 & 0.29759 \\
30 & 1.38794 & 0.3719 \\
35 & 1.43917 & 0.45377 \\
40 & 1.49479 & 0.54406 \\
45 & 1.55341 & 0.64344 \\
50 & 1.61355 & 0.75241 \\
55 & 1.67364 & 0.87124 \\
60 & 1.73207 & 1.00001 \\
65 & 1.78721 & 1.13858 \\
70 & 1.83742 & 1.28658 \\
75 & 1.8811 & 1.44342 \\
80 & 1.91673 & 1.60833 \\
85 & 1.94286 & 1.7803 \\
90 & 1.95817 & 1.95817 \\
95 & 1.96148 & 2.14058 \\
100 & 1.95177 & 2.32603 \\
105 & 1.92822 & 2.51291 \\
110 & 1.8902 & 2.69948 \\
115 & 1.83727 & 2.88394 \\
120 & 1.76925 & 3.06444 \\
125 & 1.68617 & 3.2391 \\
130 & 1.58828 & 3.40607 \\
135 & 1.47606 & 3.56353 \\
140 & 1.35023 & 3.70973 \\
145 & 1.2117 & 3.84302 \\
150 & 1.06158 & 3.96187 \\
155 & 0.90117 & 4.06491 \\
160 & 0.73192 & 4.15093 \\
165 & 0.55543 & 4.21892 \\
170 & 0.37341 & 4.26807 \\
175 & 0.18765 & 4.2978 \\
180 & 0 & 4.30775 \\
\hline & & \\
\hline
\end{tabular}

(b) C2 Materials $-K_{r}^{\max }=41,952$

Table 4.1: Displacement Field - Maxımum Applied Displacements 


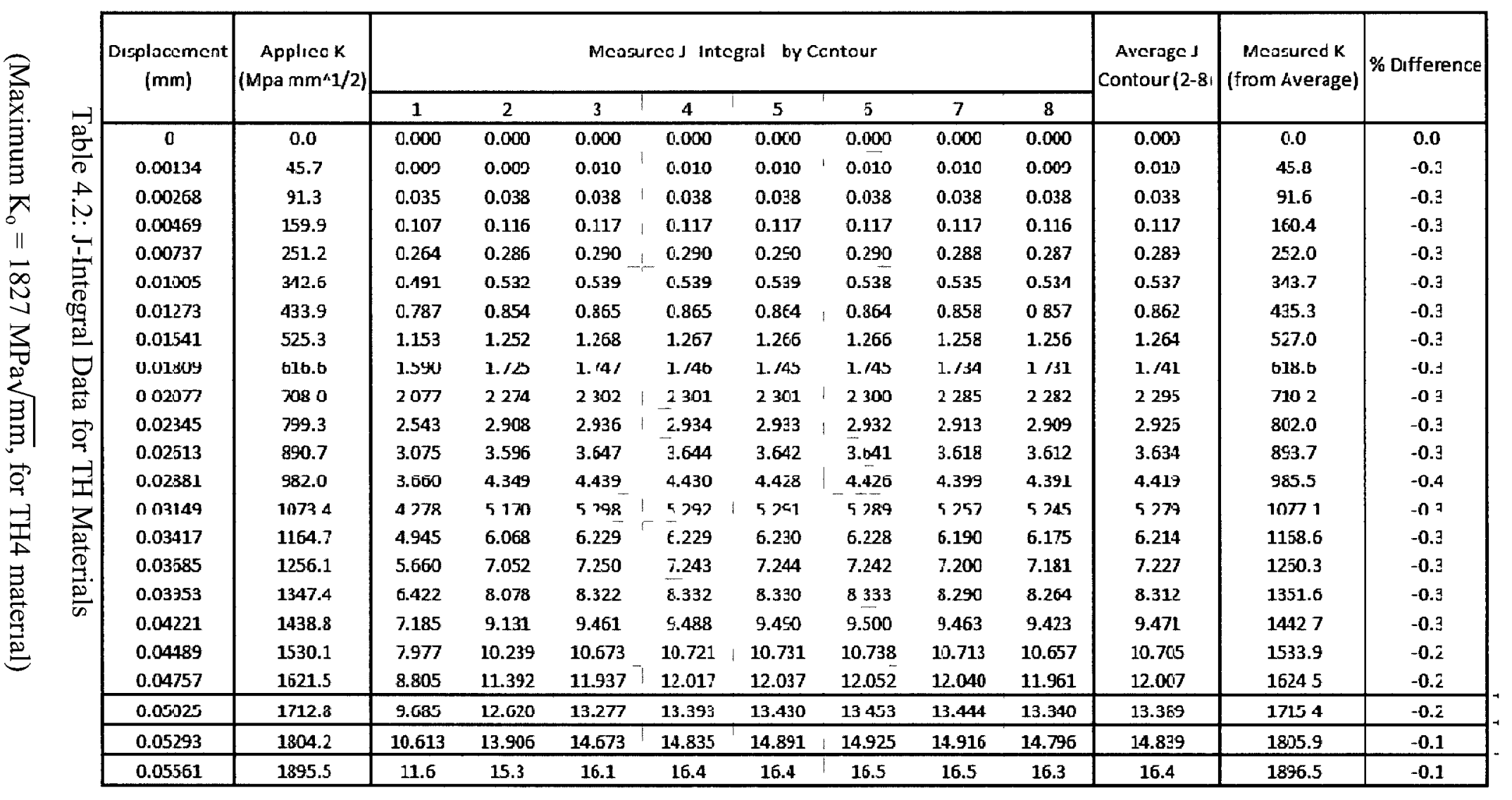




\begin{tabular}{|c|c|c|c|c|}
\hline Case & TH1 & TH2 & TH3 & TH4 \\
\hline$\hat{\mathrm{o}} / \sigma_{\mathrm{y}}$ & 3.0 & 3.5 & 3.6 & 3.75 \\
\hline $\begin{array}{c}\Gamma_{\mathrm{o}} \\
(\mathrm{MPa} \cdot \mathrm{mm})\end{array}$ & 12.150 & 14.175 & 14.580 & 15.188 \\
\hline $\begin{array}{c}\mathrm{K}_{\mathrm{o}} \\
(\mathrm{MPa} \sqrt{\mathrm{mm}})\end{array}$ & 1634.1 & 1765.0 & 1790.1 & 1827.0 \\
\hline $\mathrm{R}_{\mathrm{o}}(\mathrm{mm})$ & 0.787 & 0.918 & 0.944 & 0.984 \\
\hline
\end{tabular}

(a)

\begin{tabular}{|c|c|c|c|}
\hline Case & C2_1 & C2_2 & C2_3 \\
\hline $\begin{array}{c}\hat{\mathrm{o}} / \sigma_{\mathrm{y}} \\
\Gamma_{\mathrm{o}} \\
(\mathrm{MPa} \cdot \mathrm{mm})\end{array}$ & 3.5 & 3.75 & 4.0 \\
\hline $\begin{array}{c}\mathrm{K}_{\mathrm{o}} \\
(\mathrm{MPa} \sqrt{\mathrm{mm}})\end{array}$ & 2538.1 & 2627.1 & 2713.3 \\
\hline $\mathrm{R}_{\mathrm{o}}(\mathrm{mm})$ & 2.060 & 2.208 & 2.354 \\
\hline
\end{tabular}

(b)

Table 4.3: Model Parameters - (a) TH Materials, (b) C2 Materials 


\begin{tabular}{|c|c|c|c|c|c|c|c|c|c|c|c|c|c|c|c|c|}
\hline \multirow{2}{*}{$\begin{array}{c}\text { Applied } \\
\text { Displacement } \\
(\mathrm{mm})\end{array}$} & \multirow{2}{*}{$\mathrm{Kr} / \mathrm{Ko}$} & \multicolumn{15}{|c|}{ Traction Stresses in Cohesive Elements (by element number) (MPa) } \\
\hline & & 1 & 2 & 3 & 19 & 30 & 54 & 82 & 111 & 137 & 138 & 139 & 140 & 141 & 142 & 143 \\
\hline 0.0000 & 00000 & 0.00 & 0.00 & 0.00 & 000 & 000 & 0.00 & 000 & 000 & 0.00 & 000 & 0.00 & 000 & 0.00 & 0.00 & 0.00 \\
\hline 00166 & 03456 & 1792.89 & 1549.57 & 1030.00 & 309.94 & 24795 & 176.42 & 143.05 & 12874 & 114.44 & 11444 & 11444 & 114.44 & 109.68 & 104.90 & 10490 \\
\hline 0.0479 & 09999 & 735.03 & 105844 & 1392.53 & 109195 & 81539 & 553.12 & 43869 & 37193 & 324.25 & | 324.25 & 324.24 & 32902 & 32902 & 314.72 & 305.18 \\
\hline 0.0581 & 12118 & 0.15 & 19048 & 563.80 & 1411.44 & 1082.43 & 3| $720.02 \mid$ & 553.13 & 462.53 & 405.31 & 405.31 & 405.31 & 410.08 & 405.31 & 391.01 & 371.93 \\
\hline 0.0581 & 12124 & 0.00 & 188.28 & 560.02 & 1411.44 & 1082.43 & 3. 720.02 & 553.13 & 462.52 & 410.07 & 405.31 & 405.31 & 410.08 & 405.31 & 391.01 & 376.70 \\
\hline 0.0598 & 12473 & 0.00 & 0.00 & 17900 & 1697.54 & 1173.02 & 76771 & 581.73 & 486.37 & 419.62 & 419.62 & 424.39 & 429.15 & 419.62 & 405.32 & 391.00 \\
\hline 0.0604 & 12591 & 0.00 & 0.00 & 0.00 & 1614.40 & 1316.08 & 810.63 & 600.81 & 495.91 & 429.15 & 429.15 & 429.15 & 433.91 & 429.16 & 414.85 & 400.54 \\
\hline 0.0604 & 12598 & 0.00 & 0.00 & 0,00 & 0.00 & 1499.83 & $|1015.67|$ & 662.80 & 519.74 & 438.69 & 43869 & 438.69 & 443.46 & 438.69 & 424.39 & 410.08 \\
\hline 00604 & 12605 & 0.00 & 0.00 & 0,00 & 0.00 & 0.00 & 1530.65 & 796.31 & 581.74 & 476.84 & 476.84 & 48161 & 48637 & 476.84 & 462.53 & 443.46 \\
\hline 0.0605 & 12612 & 0.00 & 0.00 & 0.00 & 0.00 & 0.00 & 000 & 1311.31 & 729.55 & 562.67 & 557.90 & 557.89 & 562.67 & 553.13 & 534.07 & 510.22 \\
\hline 0.0605 & 12619 & 0.00 & 0,00 & 0.00 & $=0,00$ & 0.00 & 0.00 & 0.00 & 1239.78 & 748.63 & 739.10 & 734.33 & 739.09 & 724.80 & 691.42 & 65805 \\
\hline 0.0605 & 12626 & 0.00 & 0.00 & 0,00 & 0.00 & 0.00 & 0.00 & 0.00 & 000 & 1425.74 & | 1382.83 & 1344.69 & 1306.53 & 1254.10 & 1187.33 & 1115.80 \\
\hline 0.0606 & 12633 & 0.00 & 0.00 & 000 & $0: 00$ & ${ }^{*} 0.00$ & 0.00 & 0.00 & 0.00 & 0.00 & 74.20 & 239.30 & 443.78 & 640.17 & 832.54 & 1026.70 \\
\hline 0.0607 & 12661 & 0.00 & $\therefore 0.00$ & 0.00 & 0.00 & 0.00 & 0.00 & 0.00 & 0.00 & 0.00 & 0.00 & 49.35 & 218.84 & 424.87 & 622.36 & 854.07 \\
\hline 0.0608 & 12675 & 0.00 & 0,00 & 0.00 & 0,00 & 0.00 & 000 & 0.00 & 0.00 & 0.00 & 0,00 & 0.00 & 130.33 & 331.61 & 550.79 & 777.19 \\
\hline 0.0614 & 12800 & 0.00 & 0,00 & 0,00 & 0.00 & 0.00 & 0.00 & $0: 00$ & 0.00 & $-0,00$ & 0.00 & 0.00 & 0.00 & 63.78 & 384.95 & 633.56 \\
\hline 0.0616 & 12856 & 0.00 & 0.00 & 0.00 & 0.00 & 0.00 & 0.00 & 0,00 & 0.00 & 0.00 & 0.00 & 0.00 & 0.00 & 0.00 & 35582 & 593.65 \\
\hline 0.0641 & 13379 & 0.00 & 0.00 & 0.00 & 0.00 & 0.00 & 000 & 0.00 & 0.00 & 0.00 & $0.00^{\prime-~}$ & 0.00 & 0.00 & $0: 00$ & 0.00 & 136.47 \\
\hline \multirow[t]{3}{*}{0.0643} & 13414 & 0.00 & 0.00 & 0.00 & 0.00 & 0.00 & $0: 00$ & 0.00 & 0.60 & 0,00 & 0.00 & 0.00 & 0.00 & 0.00 & 0.00 & 0.00 \\
\hline & (A)/RO & 0381 & 0,0762 & 0.114 & 0.7242 & 1.1435 & 2.0584 & 3.12 & & 5.2222 & & & 5,3365 & & 5,4128 & 5.4509 \\
\hline & $R_{N} / \mathbb{R}_{10}$ & 1.2124 & 2473 & 1.2591 & 1.2598 & 1.2605 & 1.2612 & 1.2619 & 1262 & 1.2633 & 1.2661 & 1.2675 & 1.28 & 1.2856 & $\begin{array}{r}1,3379 \\
\end{array}$ & 1.3414 \\
\hline
\end{tabular}




\begin{tabular}{|c|c|c|c|c|c|c|c|c|c|c|c|c|c|c|c|c|c|}
\hline \multirow{2}{*}{$\begin{array}{c}\text { Applied } \\
\text { Displacement } \\
\text { (mm) }\end{array}$} & \multirow{2}{*}{$\mathrm{Kr} / \mathrm{Ko}$} & \multicolumn{16}{|c|}{ Traction Stresses in Cohesıve Elements (by element number) (MPa) } \\
\hline & & 1 & 5 & 10 & 15 & 20 & 25 & 30 & 35 & 75 & 76 & 111 & 113 & 115 & 117 & 119 & 148 \\
\hline 0.0000 & 0.0000 & 0.00 & 0.00 & 0.00 & 0.00 & 0.00 & 0.00 & 0.00 & 0.00 & 0.00 & 0.00 & 0.00 & 0.00 & 0.00 & 0.00 & 0.00 & 0.00 \\
\hline 0.0100 & 0.1936 & 1153.96 & 362.40 & 281.33 & 205.04 & 185.97 & 166.88 & 152.58 & 133.51 & 90.60 & 95.37 & 81.06 & 76.29 & 76.29 & 76.29 & 76.30 & 61.98 \\
\hline 0.0193 & 0.3723 & 2090.54 & 662.79 & 529.29 & 395.77 & 338.55 & 314.70 & 295.64 & 257.49 & 171.66 & 171.66 & 147.82 & 143.05 & 143.05 & 143.05 & 143.06 & 109.67 \\
\hline 0.0518 & 0.9998 & 789.19 & 1749.98 & 1468.65 & 1306.54 & 1144.41 & 991.81 & 886.92 & 810.61 & 500.67 & 495.90 & 405.30 & 400.53 & 395.78 & 391.00 & 386.25 & 314.70 \\
\hline 0.0649 & 1.2527 & 0,00 & 1940.85 & 1688.00 & 1530.65 & 1397.13 & 1244.54 & 1149.18 & 1068.12 & 658.04 & 658.04 & 524.52 & 519.74 & 510.22 & 500.68 & 500.69 & 400.55 \\
\hline 0.0843 & 1.6276 & 0.00 & 0,00 & 1882.53 & 1850.13 & 1688.00 & 1583.10 & 1478.20 & 1397.13 & 939.37 & 939.37 & 729.56 & 729.55 & 715.25 & 705.71 & 700.95 & 548.37 \\
\hline 0.0975 & 1.8826 & 0.00 & 0,00 & 000 & 1846.44 & 1955.01 & 1840.60 & 1692.77 & 1583.09 & 1130.09 & 1134.87 & 896.45 & 882.15 & 877.37 & 867.83 & 853.54 & 672.33 \\
\hline 01063 & 2.0526 & 0.00 & 0.00 & Q 000 & 0.00 & 1895.24 & 195026 & 1893.05 & 174998 & 125408 & 125885 & 101566 & 996.58 & 991.82 & 972.75 & 963.21 & 767.73 \\
\hline 0.1141 & 2.2038 & 0.00 & $0.00^{2}$ & 0.00 & 0,00 & 0,00 & 1669.65 & 2039.25 & 1974.10 & 137 & 1368.52 & 1111. & 1096 & 1087.19 & 1068.12 & 1058.59 & 848.77 \\
\hline 0.1207 & 23318 & 0.00 & & 0.00 & 0,00 & 0.00 & 0,00 & 1856.04 & 2007.49 & 1463.89 & 1454.35 & 1182.56 & 117 & 1168.25 & 115 & 1139.64 & 915.57 \\
\hline 0.1245 & 24035 & 0.00 & 0.00 & -0.00 & 0.00 & 0.00 & 0.00 & 0.00 & 1617.25 & 1544.95 & 1535.42 & 124930 & 1239.78 & 1230.24 & 1206.40 & 1196.87 & 963.22 \\
\hline 0.1295 & 2.5017 & & 0.00 & 2000 & 0.00 & 0.00 & 0.00 & 0.00 & 0.00 & 1626.02 & 1611.71 & 1311.29 & 1301.76 & 1292.23 & 1268.39 & 1263.63 & 1015.68 \\
\hline 0.1306 & 25216 & $0.00^{2}$ & 0.00 & 0.00 & 0.00 & 0.00 & 0.00 & 0.00 & 0.00 & 86 & 163 & 133 & 131 & 13 & 128 & 1282.69 & 1029.97 \\
\hline 0.1310 & 2.5304 & 0,00 & & 0.00 & 000 & 0.00 & 0.00 & 0,00 & 0.00 & 1664.15 & 1649.86 & 1335.14 & 1325.60 & 1316.07 & 1287.46 & 1287.47 & 1034.76 \\
\hline 0.1315 & 2.5392 & 0.00 & 0.00 & 0.00 & 0,00 & 0.00 & 0.00 & 0,00 & 0.00 & 0.00 & 343.95 & 1630.78 & 159 & 1587.87 & 1578.33 & 1549.73 & 1249.33 \\
\hline 0.1316 & 2.5413 & & 0.00 & 0.00 & o 00 & 0.00 & 0.00 & 0.00 & 0.00 & $0.00^{2}$ & 0.00 & 1635.55 & 163 & 1611.72 & 156 & 15 & 1249.43 \\
\hline 0.1318 & 2.5445 & 000 & 0.00 & 0.00 & D.:00. & $=0.00$ & 0,00 & 0.00 & 0.00 & & 0.00 & 0.00 & 675.96 & 1537.42 & 200 & 206 & 1516.24 \\
\hline 0.1318 & 2.5455 & 0.00 & 0.00 & 0.00 & 0.00 & 0.00 & 10.00 & $0.00=$ & 0.00 & 0.00 & 0,00 & 0.00 & 0.00 & 994.48 & 1736.80 & 1979.36 & 1554.56 \\
\hline 0.1320 & 2.5497 & $=0.00$ & 0.00 & 0.00 & 0.00 & 0,00 & 0,00 & 0.00 & 0.00 & & & & 0,00 & 403.57 & 1345.78 & 1702.95 & 1549.97 \\
\hline 0.1321 & 25518 & 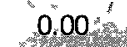 & & 000 & 000 & 0.00 & 0.00 & 0.00 & 0,00 & 000 & 0.00 & 0,00 & $0.00^{\circ}$ & 0.00 & 1225.23 & 1665.23 & 1558.78 \\
\hline 0.1325 & 2.5580 & & & 0.00 & 0.00 & 000 & 0.00 & $0.00^{\circ}$ & $0.00^{*}-$ & 0.00 & 0.00 & $=0,00$ & $0.00=$ & 0.00 & 804.01 & 1346.72 & 1579.73 \\
\hline 0.1326 & 2.5612 & 000 & 0.00 & 0.00 & 0.00 & 0.00 & 0.00 & 0.00 & 0.00 & 0.00 & 0.00 & 0.00 & 000 & 0,00 & 0.00 & 1150.02 & 1598.91 \\
\hline 0.1328 & 2.5653 & 0,00 & 0.00 & - ब.0O & 000 & 0.00 & 0.00 & 0.00 & $0.00^{d}$ & 0.0 & 0,00 & $0 ; 00$ & 1000 & $0: 00$ & 0.00 & 244.67 & 1649.29 \\
\hline 0.1329 & 2.5664 & 0.00 & 0.00 & 0.00 & 0.00 & 0.00 & 0.00 & $=0.00$ & $0.00^{2}$ & 0.00 & 000 & 0.00 & 0.00 & $\quad 0.00$ & 0,00 & 0,00 & 1634.32 \\
\hline \multirow[t]{3}{*}{0.1331} & 2.5695 & 0.00 & 000 & 0.00 & 0.00 & 0.00 & 0.00 & : $0: 00$ & 0.00 & - 0.0 & 0.00 & $0.00^{\circ}$ & 0.00 & 0.00 & 0,00 & 0.00 & 0.00 \\
\hline & / $n(x)$ & & & 0.32 & 0.45 & 0.03 & 0.81 & 309802 & 1.14 & 4.2 & 2.4831 & 3.6267 & 3.6920 & \multicolumn{2}{|c|}{$3.7573,3.8227$} & 3.8880 & 4.8355 \\
\hline & $\angle K O$ & $-1,25$ & 162 & 4.882 & 2.05 & 2.2038 & 2.3318 & 2.4035 & 2.5017 & 2.5 & 2.5413 & 2.54 .5 & 2.5455 & 2.5518 & $3 \quad 2.5612$ & 25664 & 2.5695 \\
\hline
\end{tabular}




\begin{tabular}{|c|c|c|c|c|c|c|c|c|c|c|c|c|c|c|c|c|c|}
\hline & \multirow{2}{*}{$\begin{array}{c}\text { Applied } \\
\text { Displacement } \\
(\mathrm{mm})\end{array}$} & \multirow{2}{*}{$\mathrm{Kr} / \mathrm{Ko}$} & \multicolumn{15}{|c|}{ Traction Stresses in Cohesive Elements (by element number) (MPa) } \\
\hline & & & 1 & 2 & 5 & 10 & 15 & 20 & 25 & 30 & 35 & 40 & 50 & 90 & 100 & 120 & 148 \\
\hline \multirow{21}{*}{ 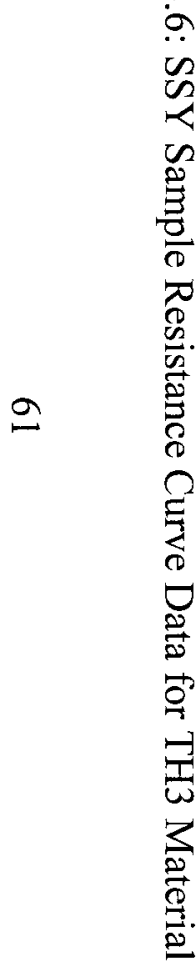 } & 0.0000 & 00000 & 0.00 & 0.00 & 000 & 0.00 & 0.00 & 0.00 & 000 & 000 & $\quad 000$ & 0.00 & 0.00 & 0.00 & 0.00 & 0.00 & 000 \\
\hline & 0.0200 & 03813 & 2149.26 & 1857.88 & 8677.10 & 548.36 & 405.30 & 352.86 & $324.25^{\prime}$ & 295.64 & 267.03 & 247.95 & 228.88 & 162.13 & 15736 & 143.05 & 119.21 \\
\hline & 0.0525 & 10001 & 798.67 & 1278.29 & 91707.09 & 1473.43 & 1316.08 & 1158.71 & | 1006 13| & \begin{tabular}{l|l}
3 & 906.00
\end{tabular} & 824.91 & 753.40 & 643.72 & 462.52 & 438.69 & 391.00 & 314.70 \\
\hline & 0.0656 & 12484 & 3.23 & 45675 & 2142.48 & 1626.01 & 1535.42 & 1397.13 & '1249.31، & $1,1158.71$ & 1082.42 & 991.82 & 863.08 & 605.58 & 567.44 & 505.45 & 405.31 \\
\hline & 0.0656 & 12494 & 0.00 & 450.79 & 2140.12 & 1630.77 & 1535.42 & 1397.13 & 125408 & 81158.72 & 1082.42 & 991.82 & 863.08 & 600.81 & 567.44 & 500.68 & 405.31 \\
\hline & 0.0740 & 1.4099 & 0.00 & 0.00 & 1583.69 & 1888.26 & 1659.40 & 1502.03 & , 1397.13 & 31301.77 & 1211.17 & 1130.10 & 1006.12 & 705.72 & 658.04 & 576.96 & 462.50 \\
\hline & 0.0883 & 1.6811 & 0,00 & 0.00 & 0.00 & 1896.41 & 189781 & 1702.30 & 1602.17 & \begin{tabular}{l|l|} 
& 1506.81
\end{tabular} & 1425.74 & 1335.14 & 1225.47 & 891.69 & 834.47 & 729.56 & 581.76 \\
\hline & 01027 & 1.9564 & $0: 00$ & $0: 00^{4}$ * & 0.00 & 0,00 & 203669 & 194071 & 184536 & 51716.62 & 1602.17 & 1525.88 & 3416.21 & 1082.42 & 102043 & 891.69 & 710.48 \\
\hline & 0.1139 & 21686 & $0.00^{x^{20 \times n}}$ & 0.00 & 0.00 & 0.00 & 0.00 & 1983.85 & 1969.33 & 3) 190736 & 1778.59 & 1678.47 & 154019 & 1215.93 & 1149.18 & 1020.43 & 820.19 \\
\hline & 0.1213 & 2.3089 & $0: 00$ & 0.00 & 0.00 & 0.00 & 0.00 & $=0: 00$ & 212798 & 8| 1983.64 & 1950.26 & 1845.36 & 1645.08 & 1306.53 & 1239.78 & 1106.26 & 89165 \\
\hline & 0.1299 & 2.4732 & 000 & 000 & 0,00 & 0.00 & 0.00 & 0.00 & 0.00 & 1881.48 & 1997.94 & 2007.48 & 31788.14 & 1401.90 & 1335.14 & 1196.86 & 97279 \\
\hline & 0.1381 & 26299 & 0.00 & $0,00^{2}$ & 0.00 & 0.00 & 0 & $0.0 \%$ & 0.00 & Dop & 1691.96 & 2086.44 & 1935.96 & 1502.04 & 1430.51 & 1282.69 & 1049.03 \\
\hline & 0.1422 & 27083 & 0.00 & $0.00 \times$ & 0.00 & 000 & 0,00 & 0.00 & & 0.00 & -0.00 & 1945.46 & 52055.17 & 1554.49 & 1482.96 & 1330.38 & 1087.21 \\
\hline & 0.1473 & 2.8040 & 0.00 & 000 & 0.00 & $40: 00$ & 0.00 & $0.00^{\circ}$ & 0.00 & $0: 00$ & 0.00 & 0.00 & 204086 & 1621.25 & 1544.95 & 1392.36 & 1125.32 \\
\hline & 0.1555 & 29618 & 0.00 & 0.00 & 0.00 & 0.00 & 0,00 & $=0.00$ & 0.00 & 0.00 & -0.00 & $=0$. & 0.00 & 1773.84 & 1673.70 & 1502.04 & 1211.15 \\
\hline & 0.1630 & 31032 & 0.00 & 0.00 & 0 & 0,00 & 0.00 & 0.00 & 0.00 & 0.00 & 0.0 & 0.0 & 0,00 & 000 & 1794.41 & 1878.74 & 141587 \\
\hline & 0.1630 & 31043 & 0.00 & & 00 & 0.00 & 0.00 & 0.00 & 0.00 & 0.00 & 0.00 & & 0.00 & $0,00^{\circ}$ & 0,00 & 1902.58 & 1583.46 \\
\hline & 0.1631 & 31053 & $-0: 00 \%$ & 0.00 & 0.00 & 0.00 & 0000 & 0.00 & 0.00 & 0.00 & 0.0 & 0.00 & 000 & 000 & 0,00 & 0.00 & 1885.56 \\
\hline & 0.1667 & 31748 & 0.00 & 0,00 & 0.00 & 0.00 & 0.00 & $\begin{array}{r}0.00 \\
\times\end{array}$ & 0.00 & 0.00 繁 & 0.00 & 0 & wot od & 0.00 & 0.00 & 0.00 & 0.00 \\
\hline & & & & & & & & & & & & & & & & & \\
\hline & & & 1.249418 & 1.4099 & 1.6811 & 1.9564 & 2,1686 & 2.3089 & 2,4732 & 2.6299 & & & 2,9618 & 3.1032 & 3.1043 & $\begin{array}{l}3.1053 \\
\end{array}$ & 3.1748 \\
\hline
\end{tabular}




\begin{tabular}{|c|c|c|c|c|c|c|c|c|c|c|c|c|c|c|c|c|}
\hline & \multirow{2}{*}{$\begin{array}{c}\text { Applied } \\
\text { Displacement } \\
(\mathrm{mm}) \\
\end{array}$} & \multirow{2}{*}{$\mathrm{Kr} / \mathrm{Ko}$} & \multicolumn{14}{|c|}{ Traction Stresses in Cohesive Elements (MPa) } \\
\hline & & & 1 & 5 & 10 & 20 & 30 & 40 & 50 & 60 & 70 & 80 & 90 & 110 & 130 & 148 \\
\hline & 0.0000 & 00000 & 0.00 & 0.00 & 0.00 & 0.00 & 0.00 & 0.00 & 0.00 & 0.00 & 0.00 & 0.00 & 0.00 & 0.00 & 0.00 & 0.00 \\
\hline & 0.0209 & 3905 & 2238.34 & 710.49 & 576.97 & 371.92 & 305.18 & 257.48 & 23365 & 209.81 & 200.27 & 190.74 & 171.65 & 157.36 & 143.05 & 123.98 \\
\hline & 0.0536 & 09998 & 817.62 & 1640.31 & 1487.72 & 1173.01 & 929.84 & 767.71 & 662.80 & 591.27 & 543.59 & 495.90 & 472.07 & 29.15 & 381.47 & 32901 \\
\hline & 0.0664 & 12381 & 0,00 & 2130.72 & 1630.77 & 1406.67 & 1168.26 & 1006.13 & 872.61 & 782.01 & 705.72 & 648.50 & 610.35 & 538.83 & 481.61 & 410.09 \\
\hline & 0.0949 & 17708 & 0.00 & 0.00 & 1953.89 & 1721.38 & 1554.49 & 1387.60 & 1282.69 & 1187.32 & 110626 & 1015.66 & 958.44 & 858.31 & 758.17 & 638.98 \\
\hline & 0.1125 & 92 & 0.00 & 0.00 & 0.00 & 1940.71 & 1769.07 & 156402 & 1482.96 & 1378.05 & 130652 & 1225.47 & 1168.25 & 1049.04 & 944.14 & 791.53 \\
\hline & 01359 & 25363 & 0.00 & 0.00 & 0.00 & 0.00 & 203133 & 189781 & 1707.07 & 1611.71 & 1530.64 & 1463.89 & 1401.90 & 1287.46 & 1163.48 & 996.57 \\
\hline & 0.1543 & 2.8785 & $0.00_{x}$ & 0.00 & 0.00 & 0.00 & 0.00 & 208378 & 1969.34 & +1840.59 & $171662 !$ & 1616.48 & 1554.48 & 1440.05 & 1316.07 & 1130.09 \\
\hline & 0.1671 & 31183 & 0.00 & 0,00 & 0.00 & 000 & 0.00 & 0.00 & 2055.17 & 204086 & 1893.05 & 1778.60 & 1692.77 & 1554.48 & 1425.74 & 1239.74 \\
\hline & 0.1789 & 33380 & $0.00^{\circ}$ & 0.00 & 0.00 & 0.00 & $0.00^{\circ}$ & 0.00 & 0.00 & 2074.24 & 206 & 194 & 18 & 16 & 15 & 1335.10 \\
\hline & 0.1891 & 3.5277 & 0.00 & 0.00 & 0.00 & 0.00 & 0.00 & 0.00 & 0.00 & 0.00 & 2074.26 & 2083.78 & 1969.34 & 1788.13 & 1621.25 & 1420.92 \\
\hline & 0.1974 & 36834 & $\quad 0.00=$ & 00 & 0 & 40 & 0.00 & 0.00 & 000 & 0.00 & 0.00 & 2074.24 & 2079.01 & 1897.81 & 1730.92 & 1501.99 \\
\hline & 0.2049 & 38230 & 8.00 & 0.00 & 0.00 & 0.00 & $0: 00$ & 000 & 0.00 & 000 & 0,00 & -0.00 & 2088.55 & 2012.25 & 1835.82 & 1587.83 \\
\hline & 0.2124 & 39627 & 0.00 & 0.0 & 0.00 & 0.00 & 0.00 & 0.00 & 0.00 & 0.00 & 0.00 & 0.00 & 0.00 & 2102.85 & 1940.73 & 1678.42 \\
\hline & 0.2249 & 4.1951 & 000 & 0.00 & $0: 00$ & $0.00^{2}$ & $0.00^{\circ}$ & 0.00 & $=0.00$ & 0.00 & คคค & $0: 00$ & 0.00 & 0.00 & 2102.85 & 1876.80 \\
\hline & 0.2350 & 43838 & 000 & 0,00 & 0.00 & 0.00 & 0.00 & 0.00 & 0.00 & 0.00 & 0.00 & 0.00 & 0,00 & 0.00 & 0,00 & 2120.50 \\
\hline & \multirow[t]{3}{*}{0.2414} & 45043 & 0.00 & 0.00 & 0.00 & $0.00^{2}$ & 0.00 & & 0.00 & 0.00 & 0.00 & 0.00 & 6.00 & 0.00 & $0: 00$ & 0.00 \\
\hline & & Aa/R R & 0.0305 & 0.1525 & 0.3049 & $0.6099^{3 / 3}$ & 0.9 & 1.2198 & 54247 & 1.8297 & & 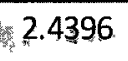 & & & 45 & 4,5132 \\
\hline & & Rr:Re & 1.2381 & 1.7708 & 2.0992 & 2.5363 & 2.8785 & $=3.1183$ & 3.3380 & 3.5277 & 3.6834 & 3.8230 & 3.9627 & 4,1951 & 4.3838 & 4.5043 \\
\hline
\end{tabular}




\begin{tabular}{|c|c|c|c|ccccccccc|}
\hline \multirow{2}{*}{$\Delta \mathrm{a}(\mathrm{mm})$} & $\begin{array}{c}\Delta \mathrm{a}-1 \\
\end{array}$ & $\begin{array}{c}\text { Measured } \\
\text { (rounded) }\end{array}$ & \multirow{2}{*}{ CTOD $(\mathrm{mm})$} & & \multicolumn{6}{|c|}{ Cpening Displacement of EL-PL Nodes (labelled by distance from original crack tip) (mm) } \\
\cline { 5 - 12 } & & & 0.5 & 0.6 & 0.7 & 1.4 & 1.5 & 1.6 & 2.2 & 2.3 & 2.4 \\
\hline 1.62 & 0.6 & 0.0163 & 1.8329 & 0.0170 & 0.0163 & 0.0154 & 0.0107 & 0.0091 & 0.0073 & 0.0002 & 0.0002 & 0.0001 \\
2.46 & 1.5 & 0.0161 & 1.9227 & 0.0216 & 0.0210 & 0.0202 & 0.0173 & 0.0161 & 0.0149 & 0.0116 & 0.0100 & 0.0083 \\
3.33 & 2.3 & 0.0170 & 1.8875 & 0.0254 & 0.0249 & 0.0242 & 0.0220 & 0.0210 & 0.0200 & 0.0181 & 0.0170 & 0.0157 \\
\hline
\end{tabular}

Table 4.8: SSY CTOA Data - TH1

\begin{tabular}{|c|c|}
\hline$\Delta \mathrm{a}(\mathrm{mm})$ & CTOA $\left.^{\circ}\right)$ \\
\hline 1.010 & 5.69 \\
1.130 & 5.33 \\
2.270 & 2.97 \\
3.350 & 2.71 \\
\hline
\end{tabular}

Table 4.9: SSY CTOA Data - TH2 


\begin{tabular}{|c|c|}
\hline$\Delta \mathbf{a}(\mathrm{mm})$ & CTOA $\left.^{\circ}\right)$ \\
\hline 1.010 & 6.61 \\
1.520 & 5.45 \\
1.760 & 5.19 \\
\hline
\end{tabular}

Table 4.10: SSY CTOA Data - TH3

\begin{tabular}{|c|c|}
\hline$\Delta \mathrm{a}(\mathrm{mm})$ & CTOA $\left.^{\circ}{ }^{\circ}\right)$ \\
\hline 1.005 & 8.06 \\
1.515 & 6.95 \\
2.025 & 6.75 \\
2.505 & 6.39 \\
3.045 & 6.52 \\
3.510 & 6.15 \\
\hline
\end{tabular}

Table 4.11: SSY CTOA Data - TH4 


\begin{tabular}{|c|c|c|c|c|c|c|c|c|c|c|c|c|c|}
\hline \multirow{2}{*}{$\begin{array}{c}\text { Applied } \\
\text { Displacement } \\
\text { (mm) }\end{array}$} & \multirow{2}{*}{$\mathrm{Kr} / \mathrm{Ko}$} & \multicolumn{12}{|c|}{ Traction Stresses in Cohesive Elements (by element number) (MPa) } \\
\hline & & 1 & 5 & 10 & 20 & 30 & 40 & 50 & 60 & 70 & 80 & 90 & 100 \\
\hline 0.0000 & 0000 & 00 & 0.0 & 0.0 & 0.0 & 0.0 & 0.0 & 0.0 & 0.0 & 0.0 & 0.0 & 0.0 & 0.0 \\
\hline 0.0192 & 0.253 & 2011.4 & 6342 & 510.2 & 3290 & 2766 & 2336 & 2098 & 1907 & 1812 & 1621 & 1621 & 152.6 \\
\hline 0.0762 & 1.000 & 926.3 & 1813.2 & 1521.1 & 14162 & 12541 & 1111.0 & 1010.9 & 906.0 & 834.4 & 762.9 & 715.2 & 681.9 \\
\hline 0.0992 & 1.301 & 0.0 & 1529.1 & 1616.5 & 1554.5 & 1435.3 & 13256 & 1258.8 & 1158.7 & 1111.0 & 1034.7 & 982.3 & 934.6 \\
\hline 0.1822 & 2.391 & 0.0 & 0.0 & 1597.7 & 18072 & 1702.3 & 16165 & 15879 & 1535.4 & 1511.6 & 1478.2 & 1454.3 & 1440.1 \\
\hline 0.1967 & 2581 & 0.0 & 0.0 & 00 & 1745.2 & 1764.3 & 1668.9 & 16403 & 15831 & 1564.0 & 1530.6 & 1502.0 & 1492.5 \\
\hline 0.2503 & 3.285 & $0 ; 0$ & 0.0 & 0.0 & 0.0 & 1764.3 & 18072 & 1759.5 & 1702.3 & 1683.2 & 1654.6 & 1630.8 & 1621.2 \\
\hline 0.3151 & 4.134 & $0,0,0$ & 0,0 & 0.0 & 0.0 & $0.0^{\circ}$ & 1814.8 & 1874.0 & 1831.0 & 1778.6 & 1759.5 & 1730.9 & 1707.1 \\
\hline 0.3447 & 4.523 & w 0,0 & 0,0 & 0,0 & 0.0 & 0.0 & 0.0 & 1818.1 & 1869.2 & 1821.5 & 1807.2 & 1773.8 & 1764.3 \\
\hline 0.3697 & 4850 & $0.0_{0}$ & 0,0 & 0,0 & 0.0 & $0^{x} .0^{\text {san }}$ & 0.0 & -0.0 & 1807.2 & 1859.7 & 1835.8 & 1812.0 & 1792.9 \\
\hline 0.3953 & 5.187 & & 0.0 & 0.0 & 0.0 & 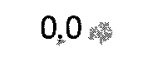 & 0.0 & 0.0 & 0.0 & 1827.0 & 1873.9 & 1835.8 & 1840.6 \\
\hline 0.4177 & 5.480 & 0.0 & $0.0^{\circ}$ & $0: 0$ & $0.0^{\circ}$ & 0.0 & 0.0 & 0.0 & 0.0 . & 0.0 & 1834.0 & 1878.7 & 1840.6 \\
\hline 04403 & 5778 & & & & & 0.0 & 00 & 0.0 & 0.0 & 0.0 & 0.0 & 18259 & 18644 \\
\hline 04623 & 6.066 & -0 & 0.0 & & 0.0 & 0.0 & 0.0 & 0.0 & 0 & 0.0 & 0.0 & 0,0 & 1835.8 \\
\hline \multirow[t]{4}{*}{04847} & 6.359 & 0.0 㒸 & $0: 0$ & 0.0 & 0.0 & 0.0 & 0.0 & 0.0 & 00 & 0.0 & 0.0 & 0.0 & 0.0 \\
\hline & $\Delta$ & 0.03 & 0.15 & 0,3 & 0.6 & 0.9 & 12 & 15 & 1,8 & 2.1 & 2.4 & 2.7 & 3 \\
\hline & $\Delta \mathrm{A} / \mathrm{R} / \mathrm{R}$ & 0.0146 & 0.0728 & 0.1456 & 0.2912 & 0.4369 & 0.5825 & 0,7281 & 08737 & 1.0194 & 1,1650 & 1.3106 & 1,4562 \\
\hline & Kiflko & 1.3011 & 2,3909 & 2.5812 & 3.2848 & 4.13444 ㄴ. & 4.5231 & $4.8503^{3}$ & 5.1873 & 5.4801 & 5.7777 & 6.0664 & 6.3592 \\
\hline
\end{tabular}




\begin{tabular}{|c|c|c|c|c|c|c|c|c|c|c|c|c|}
\hline \multirow{2}{*}{\begin{tabular}{|c|} 
Applied \\
Displacement \\
(mm)
\end{tabular}} & \multirow{2}{*}{$\mathrm{Kr} / \mathrm{Ko}$} & \multicolumn{11}{|c|}{ Traction Stresses in Cohesive Elements (by element number) (MPa) } \\
\hline & & 1 & 5 & 10 & 20 & 30 & 40 & 50 & 60 & 70 & 80 & 90 \\
\hline 0.0000 & 0.000 & 0.0 & 0.0 & 0.0 & 0.0 & 0.0 & 0.0 & 0.0 & 0.0 & 0.0 & 0.0 & 0.0 \\
\hline 0.0218 & 0.277 & 2149.9 & 793.9 & 634.2 & 410.1 & 343.3 & 286.1 & 257.5 & 231.3 & 217.0 & 197.9 & 190.7 \\
\hline 0.0789 & 1.001 & 942.1 & 1671.4 & 1525.9 & 1425.7 & 1294.6 & 1154.0 & 1056.2 & 956.1 & 884.5 & 810.6 & 762.9 \\
\hline 0.0997 & 1.263 & 0.0 & 1464.3 & 1635.5 & 1545.0 & 1444.8 & 1339.9 & 1275.5 & 1182.6 & 1125.3 & 1053.8 & 999.0 \\
\hline 0.2229 & 2.826 & 0.0 & 0.0 & 1969.2 & 1747.6 & 1742.8 & 1659.4 & 1630.8 & 1595.0 & 1576.0 & 1559.3 & 1540.2 \\
\hline 0.2746 & 3.481 & 0.0 & 0.0 & 0.0 & 1771.4 & 1797.7 & 1759.5 & 1699.9 & 1668.9 & 1654.6 & 1647.5 & 1623.6 \\
\hline 0.3912 & 4.959 & 0.0 & 0.0 & 0.0 & 0.0 & 1788.1 & 1885.9 & 1783.4 & 1790.5 & 1771.4 & 1761.9 & 1738.1 \\
\hline 0.4379 & 5.551 & 0.0 & & 0.0 & 0.0 & 0.0 & 1907.4 & 1845.4 & 1823.9 & 1797.7 & 1792.9 & 1788.2 \\
\hline 0.5183 & 6.570 & 0.0 & 0.0 & 0.0 & 0.0 & 0.0 & 0.0 & 1812.0 & 1955.1 & 1862.0 & 1843.0 & 1843.0 \\
\hline 0.5489 & 6.958 & 0.0 & & 0.0 & 0.0 & 0.0 & $0.0 \ldots$ & 00 & 1869.2 & 1914.5 & 1874.0 & 1857.3 \\
\hline 0.6167 & 7.818 & 0.0 & 0.0 & 0.0 & 0.0 & 0.0 & 0.0 & 0.0 & 0.0 & 1871.6 & 1936.0 & 1912.1 \\
\hline 0.6416 & 8.133 & 0.0 & 0.0 & 0.0 & 0.0 & $0: 0$ & 0,0 & 0.0 & 0.0 & 0.0 & 1885.9 & 1936.0 \\
\hline 0.6818 & 8.642 & 0.0 & 0.0 & 0.0 & 0.0 & 0.0 & 00 & 0.0 & 00 & 0.0 & 0.0 & 1881.1 \\
\hline \multirow[t]{5}{*}{0.7177} & 9.098 & 0.0 & 0.0 & $0.0^{x^{x} x^{m+x}}$ & 0.0 & 0.0 & $0.0^{2}$ & 0.0 & 0.0 & 0.0 & 0.0 & 0.0 \\
\hline & \\
\hline & $\Delta a$ & 0.03 & 0.15 & 0.3 & 0.6 & 0.9 & 1.2 & 1.5 & 1.8 & 2.1 & 2.4 & 2.7 \\
\hline & $\Delta \mathrm{a} / \mathrm{Ro}$ & 0.0136 & 0.0680 & 0.1359 & 0.2718 & 0.4077 & 0.5437 & 0.6796 & 0.8155 & 0.9514 & 1.0873 & 1.2232 \\
\hline & $\mathrm{Kr} / \mathrm{Ko}$ & 1.2633 & 2.8258 & 3.4809 & 4.9589 & 5.5508 & 6.5697 & 6.9578 & 7.8178 & 8.1329 & 8.6422 & 9.0979 \\
\hline
\end{tabular}




\begin{tabular}{|c|c|c|c|c|c|c|c|c|c|c|c|c|c|c|c|c|}
\hline \multirow{2}{*}{\multicolumn{2}{|c|}{ 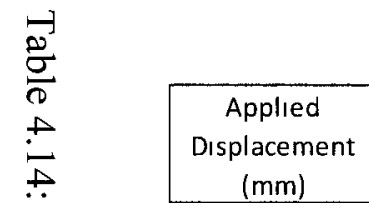 }} & \multirow{2}{*}{$\mathrm{Kr} / \mathrm{Ko}$} & \multicolumn{14}{|c|}{ Traction Stresses in Cohesive Elements (by element number) (MPa) } \\
\hline & & & 1 & 3 & 5 & 6 & 9 & 12 & 14 & 16 & 29 & 43 & 62 & 71 & 72 & 73 \\
\hline \multirow{2}{*}{$\underset{\sim}{\mathscr{L}}$} & 0.0000 & 0.000 & 0.0 & 00 & 0.0 & 00 & 00 & 00 & 0.0 & 00 & 00 & 0.0 & 00 & 00 & 0.0 & 0.0 \\
\hline & 0.0251 & 0.308 & 2292.8 & 1387.9 & 848.8 & 832.1 & 743.9 & 624.7 & 538.8 & 526.9 & 376.7 & 305.2 & 250.3 & 233.7 & 231.3 & 2289 \\
\hline \multirow{4}{*}{ 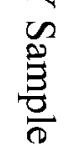 } & 0.0816 & 1.001 & 969.8 & 1934.8 & 15880 & $177 \underline{3} 8$ & 1585.5 & 15330 & 1552.1 & 1497.3 & 1339.9 & 1170.6 & 984.7 & 922.7 & 913.1 & 898.8 \\
\hline & 0.1020 & 1.251 & 0,0 & 15243 & 1304.6 & 18668 & 1716.6 & 1626.0 & 1652.2 & 1609.3 & 1463.9 & 1351.8 & 1194.5 & 1139.7 & 1130.1 & 11182 \\
\hline & 0.1170 & 1.435 & $0: 0$ & 1068.9 & 12304 & 1919.1 & 1688.0 & 1669.0 & 16951 & 1649.8 & 15307 & 14305 & 1311.3 & 1270.8 & 1258.8 & 12422 \\
\hline & 0.2127 & 2.610 & 0.0 & 0,0 & 20798 & 21483 & 1566.7 & 18358 & 17977 & 1821.5 & 1711.9 & 1657.0 & 1576.0 & 1559.3 & 1556.9 & 15545 \\
\hline \multirow{3}{*}{ 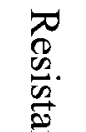 } & 0.2419 & 2.968 & 0.0 & 0.0 & 3321 & 11729 & 22153 & 1742.8 & 1764.3 & 1833.4 & 1747.6 & 16832 & 1616.5 & 1604.6 & 1599.8 & 15998 \\
\hline & 02484 & 3.048 & 0,0 & 0.0 & 0.0 & 7373 & 22897 & 1690.4 & 18072 & 1857.3 & 1766.7 & 1692.8 & 1628.4 & 1611.7 & 1609.3 & 1609.3 \\
\hline & 0.2699 & 3.313 & 0.0 & 0.0 & 0.0 & 0.0 & 21515 & 15285 & 1919.3 & 1843.0 & 1790.5 & 17119 & 1649.9 & 1642.7 & 1635.6 & 16308 \\
\hline \multirow{3}{*}{$\begin{array}{l}\vec{\Xi} \\
\stackrel{8}{8} \\
\Omega\end{array}$} & 0.4024 & 4.938 & $0.0^{\circ}$ & 0.0 & $0,0, \infty$ & 0.0 & 0.0 & 1595.1 & 1103.9 & 1425.9 & 1802.4 & 18859 & 17691 & 17762 & 1757.1 & 1738.1 \\
\hline & 0.6267 & 7.692 & 0,0 & 0.0 & $x^{2} 0$ & 0.0 & $x, 0.0$ & , o. & 9403 & 766.4 & 1544.8 & 14593 & 1773.8 & 1869.2 & 1845.4 & 1900.2 \\
\hline & 0.6271 & 7.696 & 0.0 & 0.0 & 00 & 0.0 & 0.0 & 0.0 & 0 & $7 \overline{7.8}$ & 1108.8 & 1313.8 & 16997 & 1848.5 & 1716.4 & 1654.8 \\
\hline \multirow{2}{*}{$\sum_{\infty}^{\infty}$} & 0.6272 & 7.698 & 0.0 & 0.0 & 0.0 & 0.0 & 000 & 0.0 & 0.0 & 70.6 & 1116.0 & 13972 & 16216 & 1657.0 & 1647.4 & 1714.4 \\
\hline & 0.6300 & 7.732 & 0.0 & 0.0 & 0.0 & 0.0 & 0.0 & 0.0 & 0,0 & 0,0 & 1106.5 & 1306.6 & 1699.9 & 17690 & 1761.8 & 1826.6 \\
\hline \multirow{2}{*}{ 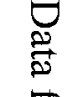 } & 08022 & 9.846 & 0.0 & 0.0 & 0.0 & 00 & 0 & & & 0.0 & 0.0 & 1082.4 & 13130 & 1497.5 & 13827 & 1420.7 \\
\hline & 0.8024 & 9.849 & 0.0 & 0.0 & 0.0 & 0.0 & $0.0^{\circ}$ & 0.0 & 0.0 & 0.0 & 0.0 & 0.0 & 1002.6 & 14887 & 1319.7 & 1146.0 \\
\hline \multirow{3}{*}{$\stackrel{\vec{O}}{\Omega}$} & 08024 & 9849 & 20.0 & 0 & 0.0 & 00 & 0.0 & 0.0 & 0.0 & 0.0 & 0.0 & 0.0 & 0.0 & 11988 & 1126.1 & 925.3 \\
\hline & 08024 & 9849 & $0, \mathrm{Q}^{2}$ & 0.0 & 0,0 & 00 & $0.0+$ & $\quad 0.0$ & 0.0 & 0.0 & 0.0 & 0.0 & 0.0 & 0.0 & 553.3 & 1473.9 \\
\hline & 08024 & 9849 & 0.0 . & 0.0 & 0,0 & 0.0 & 0.0 & $1 E 0=0.0$ & $=0.0$ & 0.0 & 0.0 & 0.0 & 0.0 & 0.0 & 0.0 & 958.2 \\
\hline \multirow{3}{*}{$\underset{\substack{\mathbb{0} \\
\stackrel{0}{0}}}{\stackrel{3}{0}}$} & & & & & & & & & & & & & & & & \\
\hline & & 这司 & $\begin{array}{l}0.03 \\
0.0127\end{array}$ & 0,09 & $0: 15$ & $0: 18$ & $\begin{array}{cc}0.27 \\
0.1147\end{array}$ & $\begin{array}{c}0.36 \\
0.1529\end{array}$ & 0.42 & $0 ; 48$ & $\begin{array}{c}0.87 \\
0.3695\end{array}$ & $\begin{array}{c}1.29 \\
0.5479\end{array}$ & 1.86 & $2: 13$ & 2.16 & $\begin{array}{l}\text { N/A } \\
\text { N/A }\end{array}$ \\
\hline & & 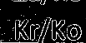 & 1.25137 & 2.610287 & 3.048326 & 3.313051 & 4.938458 & 7.691755 & 7.696468 & 7.731754 & 9.845594 & 9.848662 & 9.848674 & 9.848674 & 9.848674 & NA \\
\hline
\end{tabular}




\begin{tabular}{|c|c|}
\hline$\Delta \mathrm{a}(\mathrm{mm})$ & $\mathrm{CTOA}^{\circ}{ }^{\circ}$ \\
\hline 0.96 & 29.1 \\
1.05 & 24.1 \\
1.17 & 25.8 \\
1.26 & 25.8 \\
1.35 & 22.6 \\
1.47 & 23.7 \\
1.56 & 24.6 \\
1.65 & 19.6 \\
1.77 & 19.1 \\
1.86 & 19.5 \\
1.95 & 20.5 \\
2.07 & 18.8 \\
2.16 & 19.0 \\
2.25 & 20.1 \\
2.37 & 18.8 \\
2.46 & 19.1 \\
2.55 & 19.8 \\
2.67 & 18.6 \\
2.76 & 19.1 \\
2.85 & 20.1 \\
2.97 & 19.0 \\
3.06 & 19.3 \\
3.15 & 20.1 \\
\hline
\end{tabular}

(a) $\mathrm{C} 2 \_1$

\begin{tabular}{|c|c|}
\hline$\Delta \mathrm{a}(\mathrm{mm})$ & CTOA $\left.^{\circ}\right)$ \\
\hline 0.96 & 44.4 \\
1.05 & 41.9 \\
1.17 & 41.6 \\
1.26 & 40.8 \\
1.35 & 33.9 \\
1.47 & 32.5 \\
1.56 & 33.5 \\
1.65 & 37.5 \\
1.77 & 38.3 \\
1.86 & 38.7 \\
1.95 & 32.7 \\
2.07 & 31.2 \\
2.16 & 31.8 \\
2.25 & 38.0 \\
2.37 & 38.9 \\
2.46 & 38.0 \\
2.55 & 31.2 \\
2.67 & 29.7 \\
2.76 & 29.4 \\
2.85 & 30.4 \\
2.97 & 31.3 \\
\hline & \\
\hline
\end{tabular}

(b) $\mathrm{C} 2 \_2$

\begin{tabular}{|c|c|}
\hline$\Delta \mathrm{a}(\mathrm{mm})$ & CTOA $\left.^{\circ}\right)$ \\
\hline 1.2900 & 80.6976 \\
1.8600 & 66.6778 \\
2.1300 & 60.5626 \\
2.1600 & 60.7935 \\
\hline
\end{tabular}

(c) $\mathrm{C}_{-} 3$

Table 4.15: SSY CTOA Data - C2 Materials 

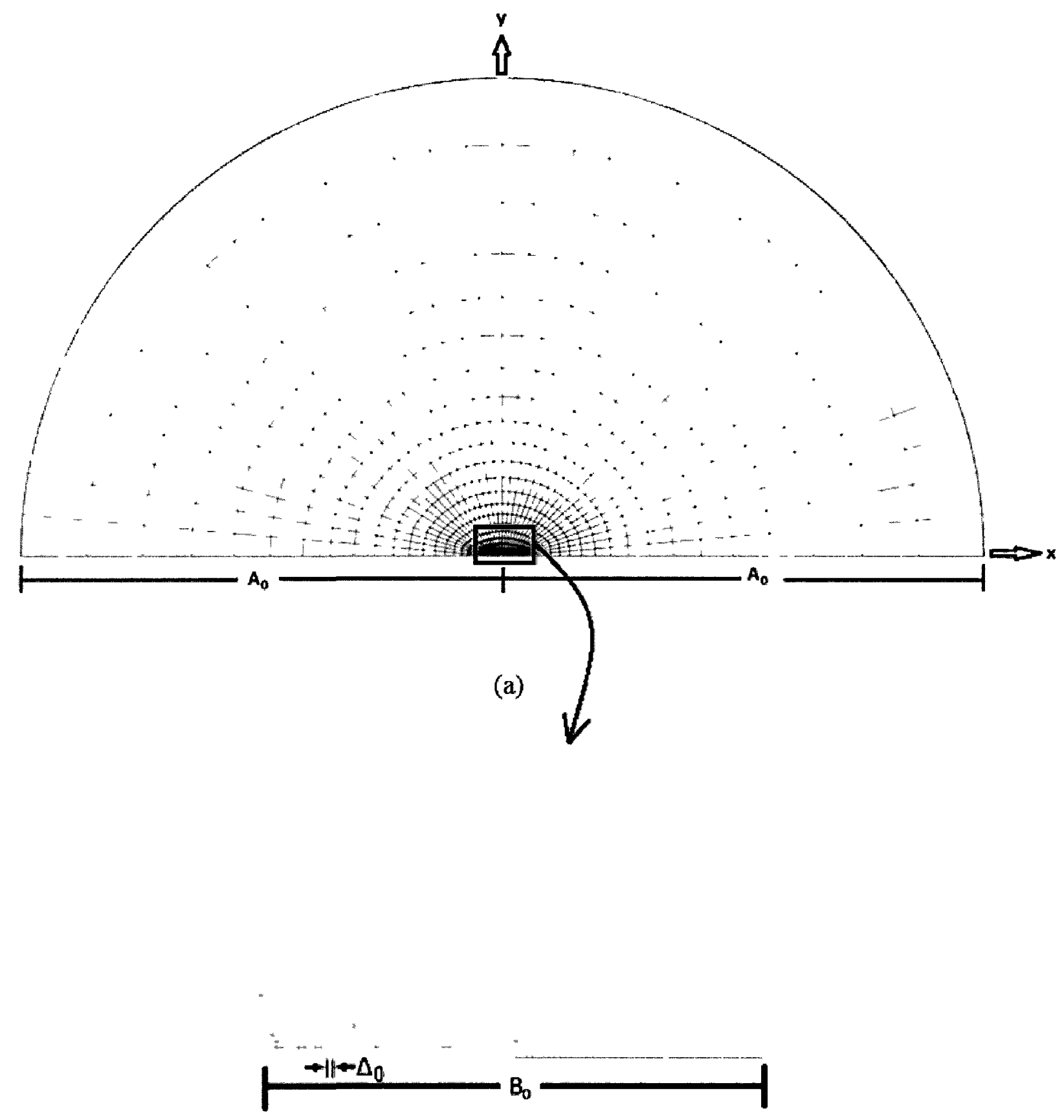

(b)

Figure 4.1: SSY Model - Geometry 


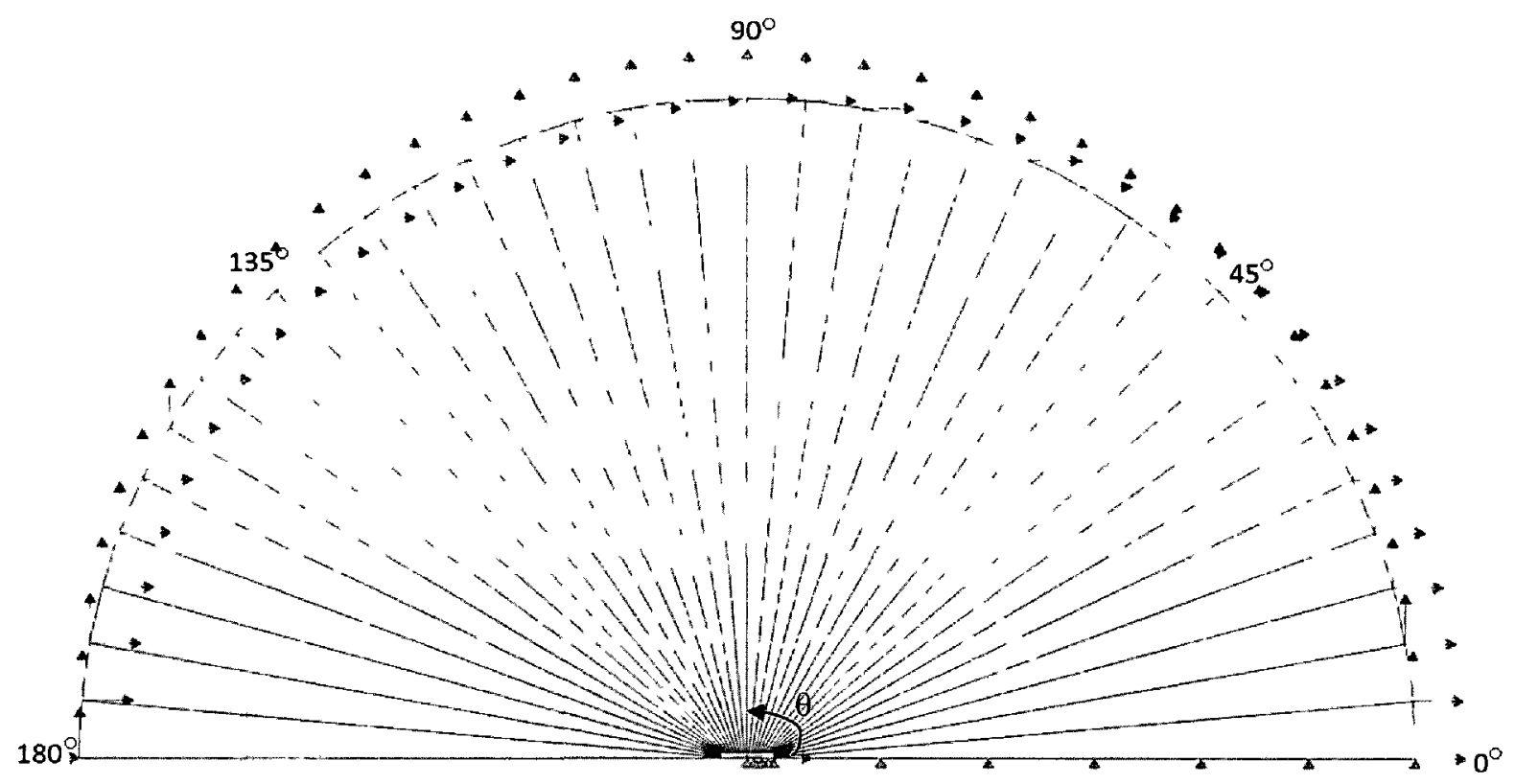

Figure 4.2: Loading Conditions

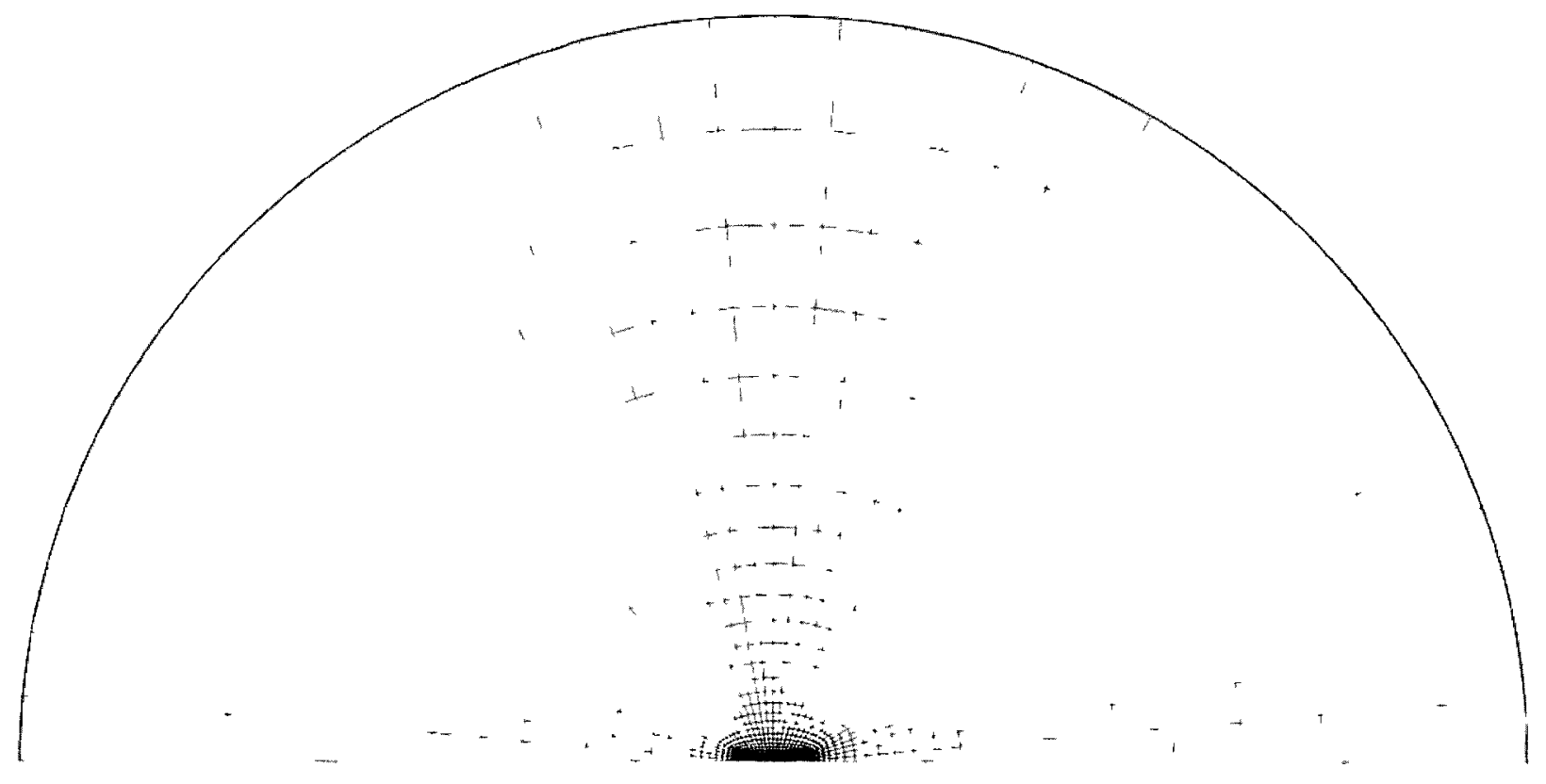

Figure 4.3: Mesh Design - Full Model 


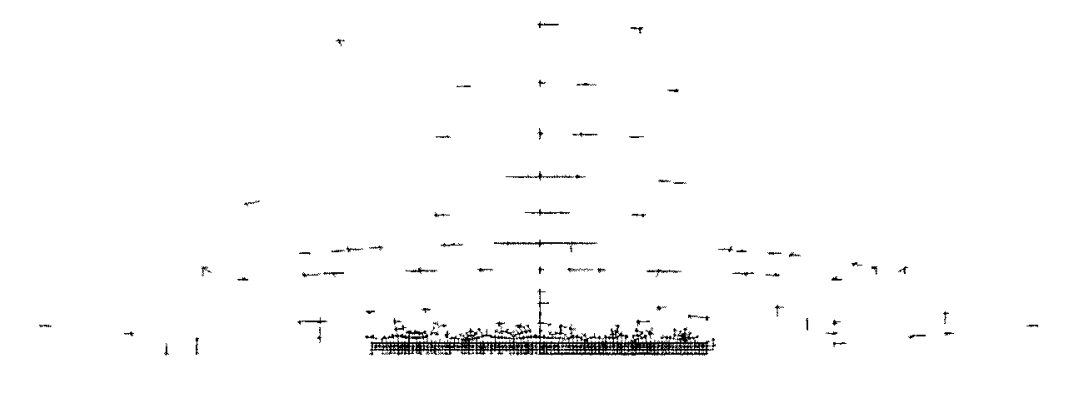

(a)

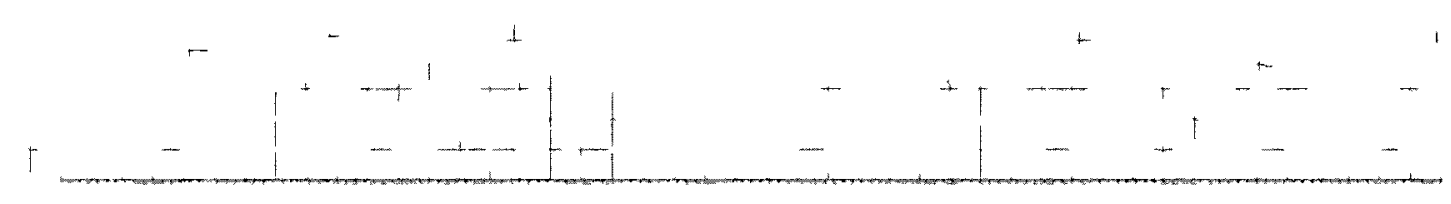

(b)

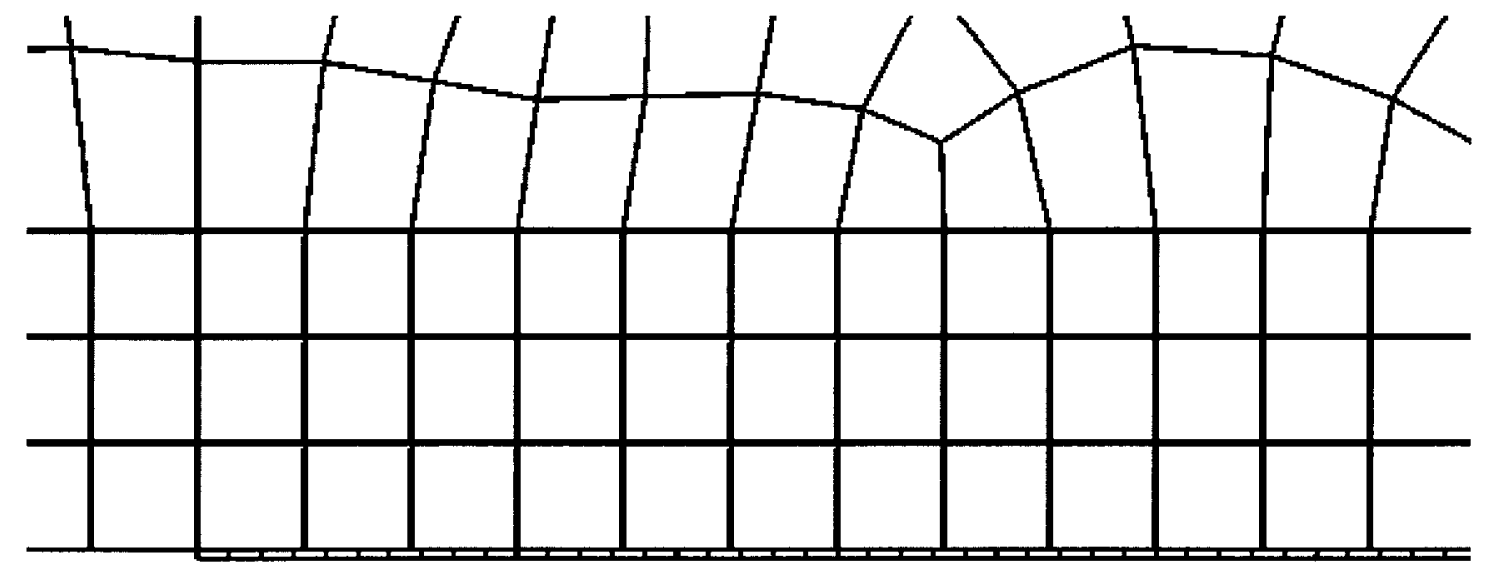

(c)

Figure 4.4 (a) - (c) C Crack Tip Mesh Desıgn 


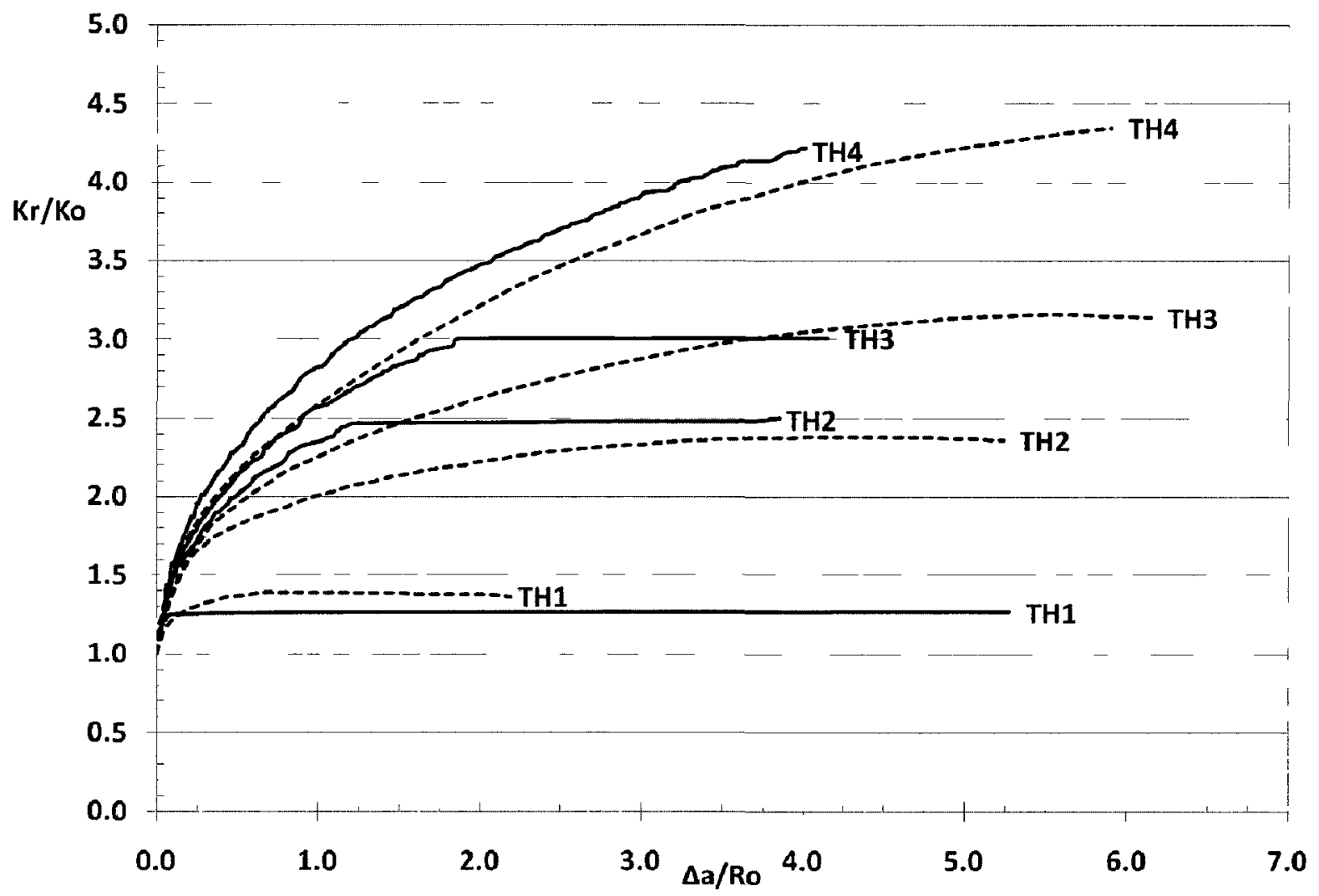

Figure 4.5 : SSY- Normalized Resistance Curves - TH Materials and With Literature Data (- ) Present work, (-----) Tvergaard and Hutchinson, 1992 


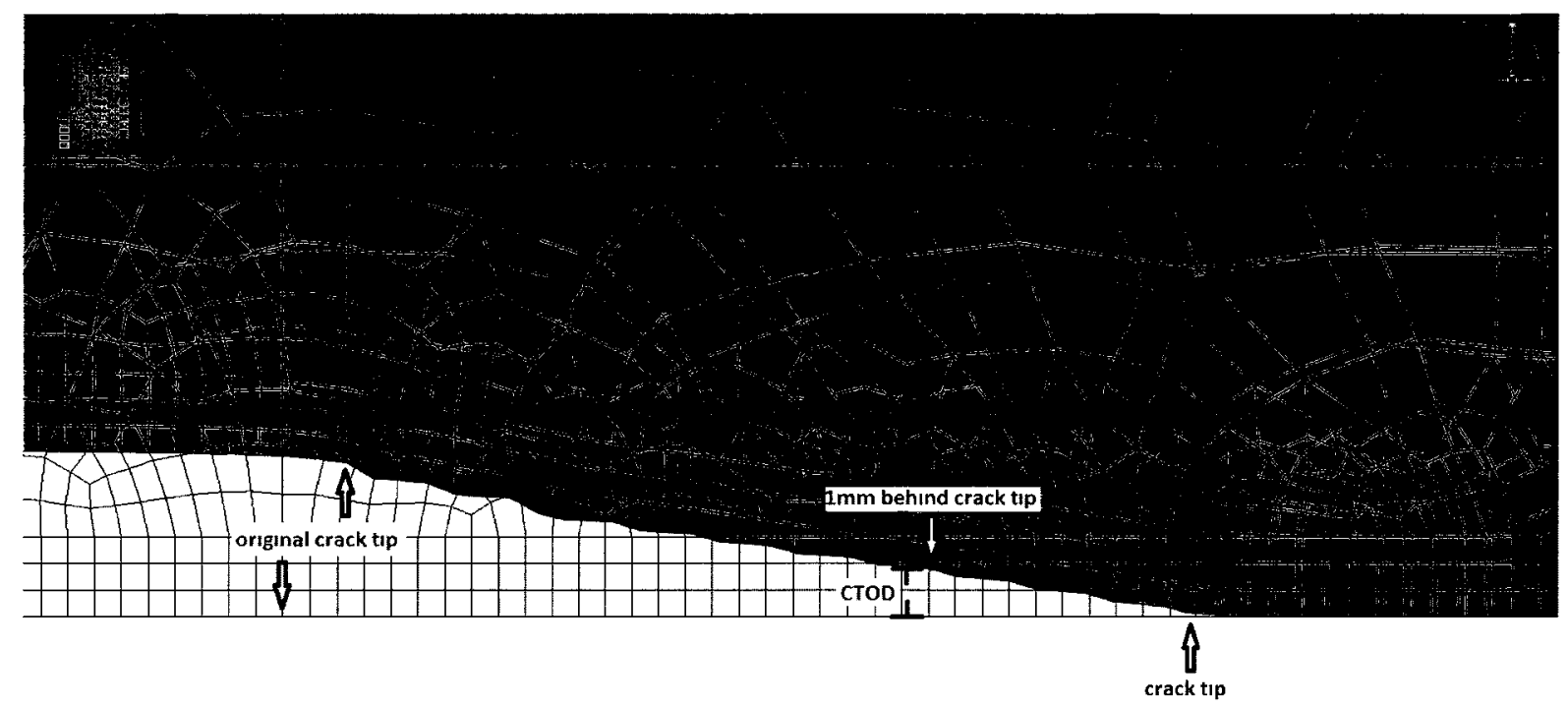

Figure 4.6 Measuring CTOA from FE Results

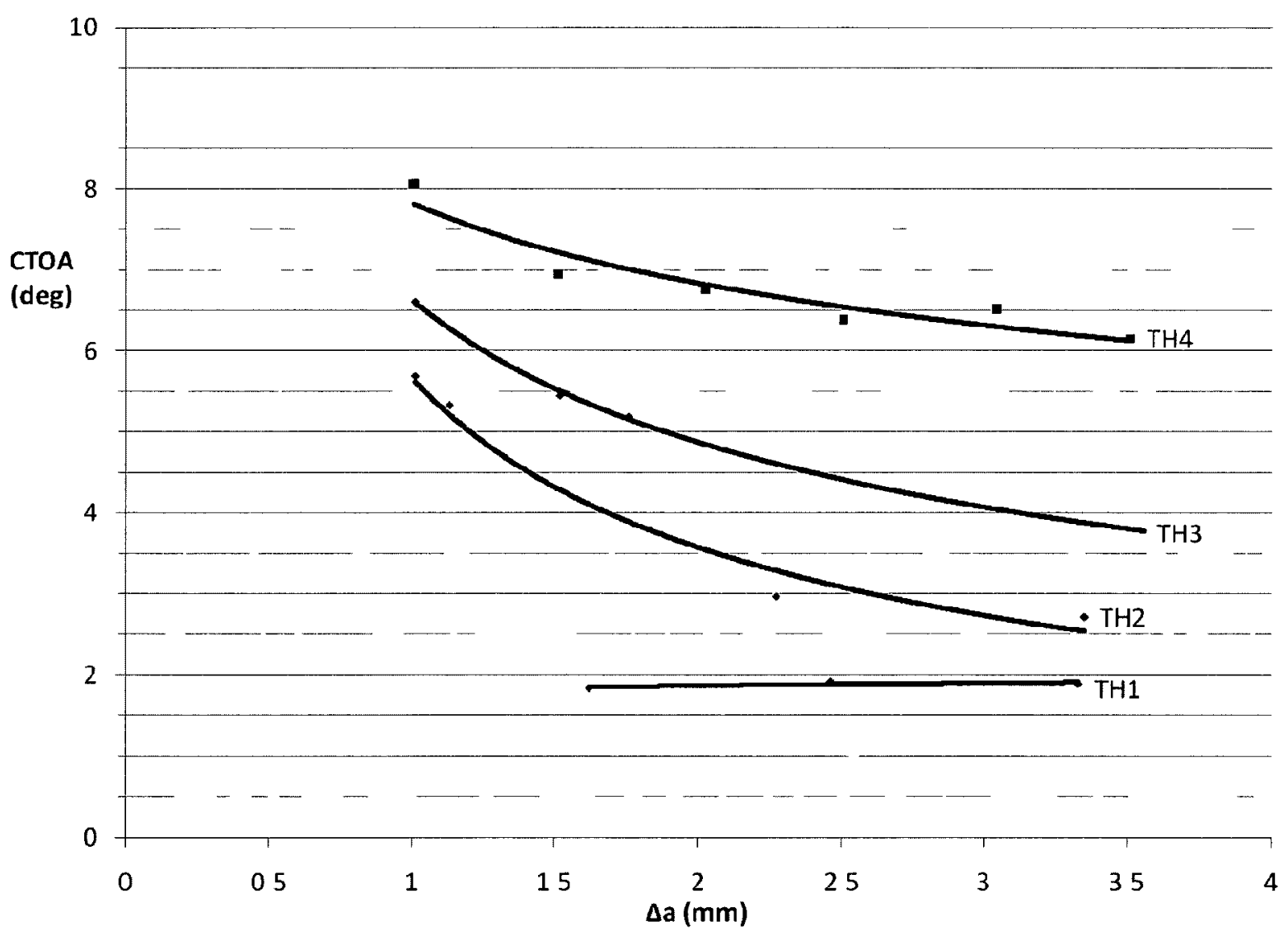

Figure 4.7 CTOA Data - SSY Model - TH Materials 


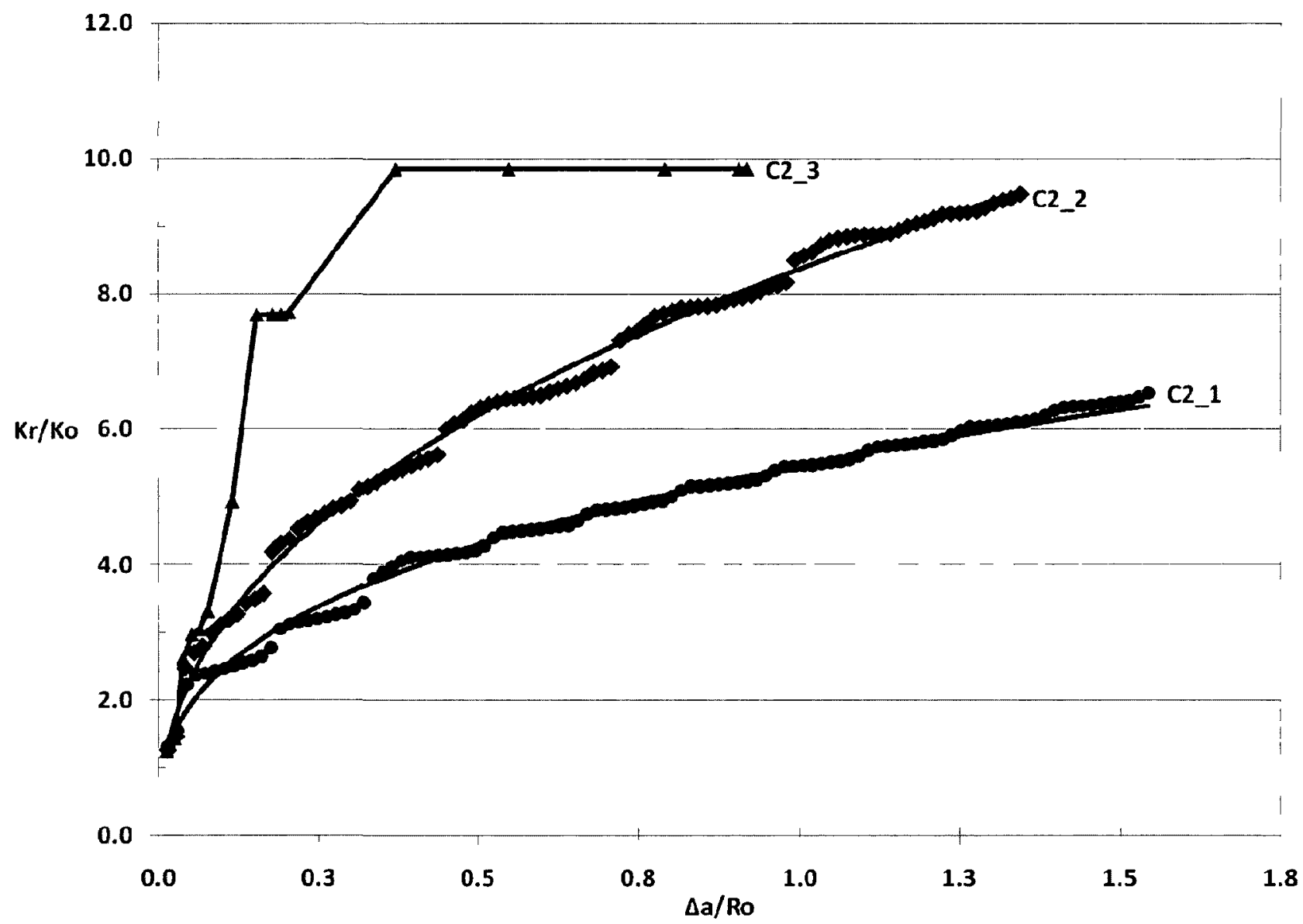

Figure 4.8 : SSY- Normalized Resistance Curves - C2 Materials 


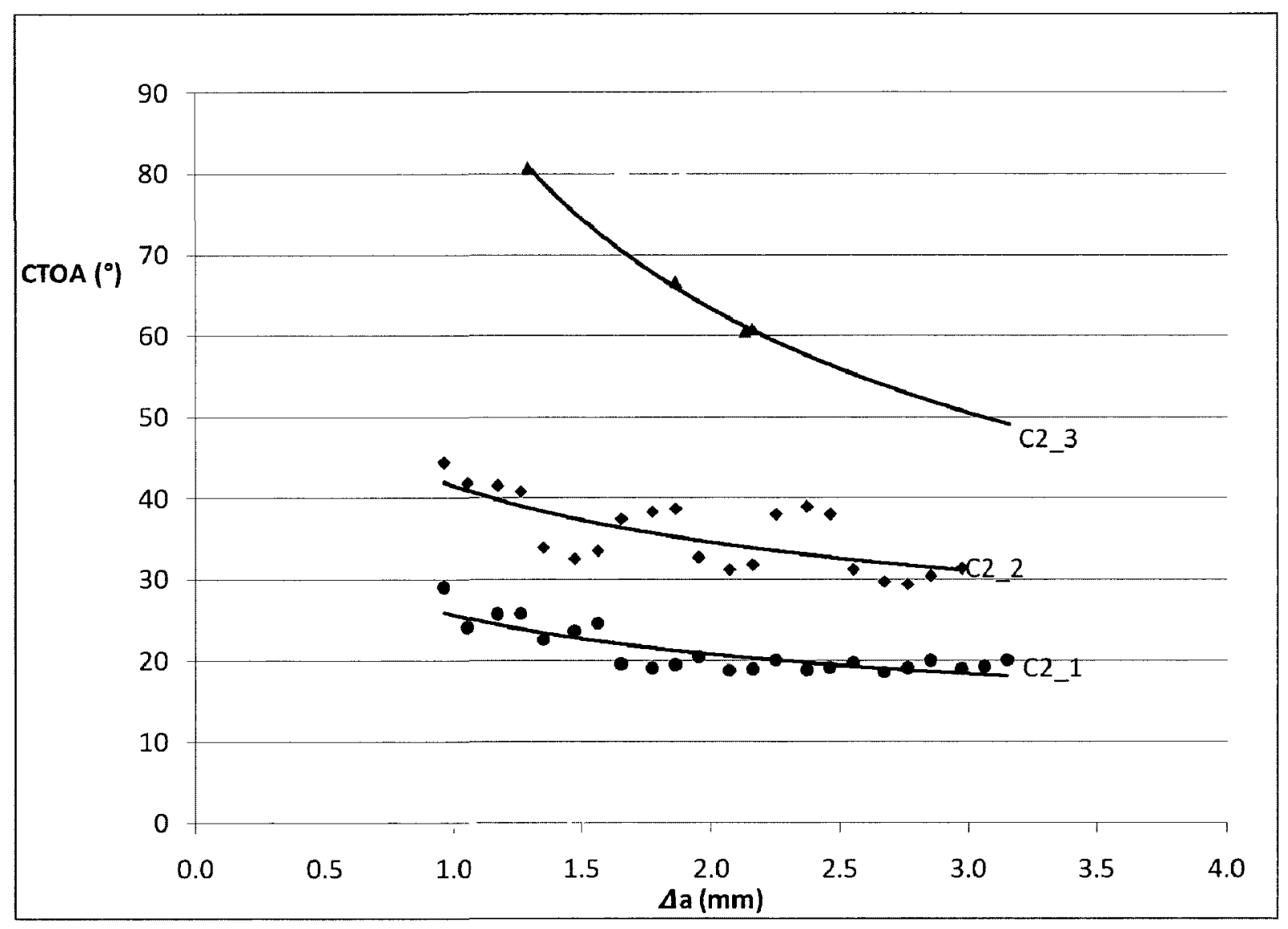

Figure 4.9 : CTOA Data - SSY Model - C2 Materials 


\section{Chapter 5}

\section{FE Simulation of Drop-Weight Tear Tests}

This chapter presents the finite element simulation of drop-weight tear tests, DWTT, using cohesive zone modeling. The computational model will be defined, including geometry, loading conditions, and mesh design. The same materials sets used in Chapters 3 and 4 will be used in this Chapter.

CANMET-MTL has performed experimental drop-weight tear tests on samples flattened and machined from a section of X70 pipeline steel (Xu, 2010a). This material is referred to in the present work as C2 steel. The DWTT specimen is preferable to Charpy and other test specimens due to its long ligament length which allows for more time to reach steady-state fracture propagation conditions. It is also relatively easy to machine. CANMET has provided load (P) vs. load-line displacement (LLD) data, as well as CTOA measurements for this $\mathrm{C} 2$ steel (Xu et al, 2010b). The data collection techniques were discussed in Chapter 2, but the data itself will be presented here.

Two sets of simulations are performed using the DWTT model. The first set of ideal or TH materials is used as a benchmark to compare between the SSY and DWTT models. The second set of materials is modeled upon the C2 material characterized by Xu (2010a). The results of the C2 material's simulations will be compared to both the SSY simulations and the experimental observations.

\subsection{Model Specifications}

\section{Geometry}

The geometry for the experimental DWTT specimen can be found in Chapter 2. Figure 5.1 shows the geometry of the FE model that is used to represent this DWTT specimen. As with the SSY model, only a half-specimen is used due to symmetry about the crack front. In this model, the crack face is along the right side of the model, with the initial crack tip located at the bottom 
of the cohesive layer. The cohesive layer, or ligament length, is $61 \mathrm{~mm}$ long. This will allow the crack to grow much farther than the maximum growth of $4.5 \mathrm{~mm}$ from the SSY model so, it becomes much more likely that a steady-state of fracture will be achieved.

As can be seen in the figure, the machined notch is ignored for these simulations, and replaced by what is effectively a flat crack with an initial length of $15 \mathrm{~mm}$. This is done to simplify the mesh. This is an approximation and is only intended to be used as a first approach to explore the feasibility of $\mathrm{CZ}$ modeling and help calibrate the cohesive parameters.

The 'tup', which is used to apply the load to the specimen in the drop-weight test, is approximated as a displacement field with a length of $12 \mathrm{~mm}$. The anvil is approximated by constraining $6 \mathrm{~mm}$ of the bottom edge in the $\mathrm{y}$-direction.

The model thickness in Abaqus is left as the default for two-dimensional elements of $1.0 \mathrm{~mm}$. The method used to calibrate the results for the specimen's actual thickness of $13.7 \mathrm{~mm}$ is described in Section 5.2.

\section{Material Properties}

As with the SSY model, two different sets of materials were used in this model. Elastic-plastic material properties can be found in Tables 3.1 and 3.4 for the $\mathrm{TH}$ and $\mathrm{C} 2$ materials, respectively. The TH materials have a Young's Modulus of 200,000 MPa, a Poisson's ratio of 0.3, and a yield stress of $600 \mathrm{MPa}$. The C2 materials have a Young's Modulus of 195,400 MPa, a Poisson's ratio of 0.3 , and a yield stress of $576 \mathrm{MPa}$. Bilinear traction-separations laws were used again, with cohesive parameters found in Tables 3.5 and 3.8 for the TH and C2 materials, respectively. Mass densities of $7.85 \times 10^{-6} \mathrm{~kg} / \mathrm{mm}^{3}$ were used for all materials to satisfy the requirements of Abaqus/Explicit. 


\section{Loading Conditions}

Figure 5.2 describes the loading conditions for these models. The nodes at the anvil are constrained in the y-direction. The anvil length of $6 \mathrm{~mm}$ can be seen in Figure 5.1. A section of nodes was constrained rather than a single node to prevent localized mesh distortion. The length of $6 \mathrm{~mm}$ was chosen through numerical experimentation and is long enough to avoid excessive deformation at the anvil.

A ramped displacement field is used to simulate the applied displacement from the 'tup' to the nodes along the top surface over a span of $12 \mathrm{~mm}$. This length was also chosen through experimentation and is able to prevent excessive mesh deformation at the 'tup'. The maximum applied displacement (load-line displacement) varies from case to case as required for complete propagation of the crack.

The nodes along the outer (right) edge of the cohesive layer are constrained in the x-direction. The nodes along the inside (left) edge of the cohesive layer are tied to the elastic-plastic material using a surface-to-surface tie constraint, as was done in the SSY model.

\section{Mesh Design}

As with the SSY model, the DWTT model is divided into two separate parts for meshing. The main rectangular section is composed of 10,264 solid quadrilateral plane-strain elements with reduced integration (CPE4R elements). The second section is a cohesive layer along the crack front composed of 2,034 cohesive (COH2D4) elements. The two sections are joined together using surface-to-surface tie constraints. Figure 5.3 shows the mesh used for the full model. Figure 5.4 highlights the mesh refinement at the 'tup', with arrows used to show how the displacement is applied at the individual nodes. Figure 5.5 highlights the mesh refinement at the anvil, and the constraint in the y-direction. Figure 5.6 shows the mesh refinement at the crack tip at increasing levels of magnification. The cohesive layer is darkened to highlight it in the bottom section of the Figure. 
As with the SSY model, the cohesive elements have a length of $0.03 \mathrm{~mm}$ for all cases, and thicknesses of 0.01 and $0.03 \mathrm{~mm}$ for the $\mathrm{TH}$ and $\mathrm{C} 2$ cases, respectively. The thicker elements allow for a higher fracture energy in the $\mathrm{C} 2$ cases. The elastic-plastic elements tied to the cohesive layer are $0.1 \times 0.1 \mathrm{~mm}$ as in the SSY model, so there are still $31 / 3$ cohesive elements per adjacent elastic-plastic element. The mesh at the crack tip is therefore quite similar to the mesh used in the SSY simulations.

Simulations were completed in Abaqus Explicit, version 6.8-2 (Abaqus, 2008), using the CAE platform on Windows 7 computers. To simulate quasi-static conditions, dynamic, explicit steps were used with a time period of one second. Results were recorded at 5,000 - 20,000 increments, depending on the simulation, to ensure sufficient data was captured to describe the crack propagation. Typical run times for these simulations were much longer than those of the SSY model, and ranged from 1-2 hours for the TH 1 simulation to up to 24 hours for the C2_3 simulation. The increase in simulation times from the SSY models was due to the larger model (12,298 elements for DWTT model vs. 1683 elements for SSY model) and the longer cohesive zone.

\subsection{Simulation Using Plane Strain Elastic-Plastic Model}

A simulation was performed without the cohesive layer to compare to the model's load vs. loadline displacement data to experimental data provided by CANMET-MTL. The simulation was run under plane strain conditions using the elastic-plastic material properties of the C2 material, as provided by $\mathrm{Xu}(2010 \mathrm{a})$.

The experimental load vs. load-line displacement data provided by CANMET for a C2 steel is found in Table $2.1(\mathrm{Xu}, 2010 \mathrm{a})$. The data was corrected for compliance, as outlined in Section 2.3.2. Results of load vs. load-line displacement from the plane strain simulation can be found in Table 5.2. Load-line displacement values were determined by measuring the displacement of nodes at the 'tup' at each time interval of the simulation. Load values were determined by measuring the reaction force in the $y$-direction at each node with an applied displacement. These 
reaction forces were then summed, multiplied by the ratio of the thickness of the specimen (13.7 $\mathrm{mm})$ to the thickness of the model $(1.0 \mathrm{~mm})$, and doubled because they represent the reaction force of only one half of the full model. This is summarized by:

$$
P(t)=2 \cdot 13.7 \cdot \sum_{\text {Tup Nodes }} F_{y}^{\text {reaction }}(t)
$$

The results of this plane strain simulation can be seen in Figure 5.7, plotted along with the experimental data from CANMET. The plane strain simulation is seen to quite closely follow the experimental data up until almost $4 \mathrm{~mm}$ of load-line displacement. The simulation halted at this point due to numerical instability. When the simulation halted, there was reaction load of 207.1 $\mathrm{kN}$. This is only about $5 \mathrm{kN}$ below the experimental value for the same load-line displacement. The sharpness of the crack in the model could play a role in reducing the peak load achieved, but overall this is quite good agreement. Additionally, it is only a 2-dimensional model and as such is unlikely to achieve perfect agreement with the 3 -dimensional lab test.

\subsection{Simulation Results for TH Materials}

As in Chapter 4, four simulations of the DWTT model were run with the TH elastic-plastic material, with the same four sets of cohesive parameters (TH1-4) as the SSY simulations. The applied displacement at the 'tup' was set to ramp up from 0 - 4 mm over 1 second of simulation. This section presents the results of these four simulations.

\section{Load vs. Load-line Displacement Curves}

The resulting load vs. load-line displacement data for the four TH materials can be found in Table 5.3 (a) - (d). The results are plotted in Figure 5.8. As expected, all four simulations followed the same elastic curve until crack propagation began. This was expected as all four simulations have the same elastic-plastic response, and cohesive element stiffnesses. The TH1 
and TH2 materials experience little or no plastic deformation and fail almost immediately upon initiation of the crack. The TH3 material shows some plastic deformation before failure, although the crack still propagates quite rapidly with little additional applied displacement. The TH4 material shows significant amounts of plastic deformation before failure, and a much more gradual slope during propagation of the crack due to the higher fracture toughness.

\section{Crack Growth Rate}

The crack growth, $\Delta \mathrm{a}$, was measured by the same procedures described in Chapter 4 . Data regarding the crack growth vs. the load-line displacement of the TH materials can be found in Table 5.4 (a) - (d). This data is plotted in Figure 5.9. It can be seen that crack initiation occurs after load-line displacements of $0.3206,0.3616,0.3676$, and $0.3717 \mathrm{~mm}$ for TH1-4, respectively. As expected, the tougher cohesive parameters lead to an increase in the required displacement for initiation. The crack growth rates are seen to drastically vary as well. The TH1 parameters allow about $15 \mathrm{~mm}$ of propagation with the same applied displacement that initiates a crack 0.03 $\mathrm{mm}$ long in the TH4 material. The TH1, 2, and 3 materials all reach a point where propagation increases at an almost infinite rate. This signifies unstable fracture. Unlike the other three materials, the TH4 material shows signs of stable ductile fracture.

\section{Crack Tip Opening Angle}

The CTOA was measured using the same procedures described in Chapter 4. CTOA vs. $\Delta$ a data for the four TH materials can be found in Table 5.5 (a) - (d). This data is plotted in Figure 5.10. Power laws were fitted to the data in MS Excel.

The CTOA values were initially quite high, but steadily dropped towards steady-state values, as expected. Steady-state values of $2.0^{\circ}, 2.5^{\circ}, 3.0^{\circ}$, and $4.5^{\circ}$ can be estimated for TH1-TH4 respectively. These CTOA values increase with increasing toughness of the cohesive elements. 
The steady-state values are quite similar to those determined during the SSY simulations of $1.9^{\circ}$, $2.5^{\circ}, 3.5^{\circ}$, and $6^{\circ}$ for $\mathrm{TH} 1-\mathrm{TH} 4$, respectively.

\subsection{Simulation Results for C2 Materials}

Three simulations of the DWTT model were run with the C2 elastic-plastic material, with the same three sets of cohesive parameters $\left(\mathrm{C}_{-}{ }_{-}-\mathrm{C}_{2} \_3\right)$ as the SSY simulations discussed in Chapter 4. The applied displacement at the 'tup' was set to ramp up from $0-10 \mathrm{~mm}$ over 1 second of simulation. All three simulations aborted around $\sim 7 \mathrm{~mm}$ of displacement due to numerical issues. This section presents the results of these three simulations, and compares the results to the experimental data for the same C2 material, as provided by $\mathrm{Xu}$ (2010a). Comparisons are also made with the CTOA results from the SSY simulations seen in Chapter 4.

\section{Load vs. Load-line Displacement Curves}

The resulting load vs. load-line displacement data for the three $\mathrm{C} 2$ materials can be found in Table 5.6 (a) - (c). The results are plotted in Figure 5.11, along with the experimental DWTT data (Xu, 2010a). As expected, all three simulations followed the same elastic curve until crack propagation began. As the fracture toughness of the cohesive elements increased from $\mathrm{C}_{2}$ 1C2_3, the slope decreased during the crack propagation stage of the P vs. LLD curve. None of the three simulations were able to meet the peak load of the experimental data. It also appears that the crack propagation in the models is beginning at a much lower applied displacement than the experimental data shows. This could be partly explained by the higher stress concentration the crack tip would see in the model because of the shape of the initial crack. A sharp crack, as in the model, has a much higher stress concentration at the crack tip than a blunt notch would see. More realistic notch geometry would need to be used in the model to improve the numerical $P$ vs. LLD results. Additionally, the results found by Alfano (2006) show that bilinear TS laws fail more readily than equivalent trapezoidal and linear-parabolic TS laws (See Figure 2.4). The peak tractions obtained between his compact tension simulation with the bilinear TS law and 
trapezoidal law different by up to $15 \%$. Conceivably, experimentation with alternate TS law shapes could also help to improve upon these numerical results for the DWTT simulations.

\section{Crack Growth Rate}

Data regarding the crack growth vs. the load-line displacement for the C2 DWTT simulations can be found in Table 5.7 (a) - (c). This data is plotted in Figure 5.12. It can be seen that crack initiation occurs after $0.366,0.544$, and $0.564 \mathrm{~mm}$ of load-line displacement for $\mathrm{C} 2 \_1-\mathrm{C} 2 \_3$, respectively. The final crack length when the simulations aborted was $12.9,7.6$, and $4.7 \mathrm{~mm}$ for C2_1-C2_3, respectively.

At an applied load-line displacement of $6 \mathrm{~mm}$, the crack has reached $\sim 11.5,6$, and $4 \mathrm{~mm}$ of extension for $\mathrm{C} 2 \_1-\mathrm{C} 2 \_3$, respectively. This is a significant reduction in crack growth rate considering the peak traction increases only from $3.5 \sigma_{y}-4.0 \sigma_{y}$, and the fracture energy increases by just $14.3 \%$ from $\mathrm{C}_{2} \_1-\mathrm{C} 2 \_3$. It is obvious from Figure 5.12 that all three materials undergo stable ductile fracture.

\section{Crack Tip Opening Angle}

CTOA vs. $\Delta$ a data for the three C2 materials can be found in Table 5.8 (a) - (c). This data is plotted in Figure 5.13. Power laws were fitted to the data in MS Excel. The trendline for the C2_3 material was extended forwards by 1 period.

As expected, the CTOAs were initially quite high, but began to steadily drop down towards steady-state values after a small amount of propagation. Furthermore, as the toughness increased from $\mathrm{C} 2 \_1$ to $\mathrm{C} 2 \_3$, the CTOA values increased also. A steady-state CTOA of $\sim 14^{\circ}$ was found for the $\mathrm{C} 2 \_1$ material. The $\mathrm{C} 2 \_2$ and $\mathrm{C} 2 \_3$ simulations did not achieve steady-state CTOAs, but using the trendlines, steady-state CTOAs of $20^{\circ}$ and $26^{\circ}$ can be estimated for C2_2 and C2_3, 
respectively. CTOA values after $1 \mathrm{~mm}$ of propagation start at $26.5^{\circ}, 39.1^{\circ}$, and $51^{\circ}$ for $\mathrm{C} 2 \_1$ C2_3 respectively. These are similar but lower than the values after about $1 \mathrm{~mm}$ crack propagation in the SSY model which were $29.1^{\circ}, 44.4^{\circ}$, and $80.7^{\circ}$ for C2_1-C2 3 respectively (See Table 4.15). This is expected as the SSY simulations were unable to reach steady-state due to the short length of the cohesive zone.

$\mathrm{Xu}$, et al, (2010) have quoted the CTOA for the C2 material as $18.5^{\circ}$, as measured optically, and $12.4^{\circ}$, as calculated by the simplified single-specimen method. The CTOA values measured in these simulations are therefore slightly higher than these experimental observations.

\subsection{Conclusions}

DWTT simulations were carried out for two different sets of materials. A plane strain elasticplastic analysis was performed for the C2 material and the simulation results were compared to the experimental data. Following this, simulations were performed using the four TH materials, and $\mathrm{P}$ vs. LLD, $\triangle \mathrm{a}$ vs. LLD, and CTOA vs. $\triangle \mathrm{a}$ were calculated and plotted. The steady-state CTOA measurements for the TH materials were compared to the measurements from the SSY simulations and there was found to be good agreement between the two simulations. Steady-state CTOAs from the DWTT simulations with the TH materials ranged from $2^{\circ}-4.5^{\circ}$.

The tougher $\mathrm{C} 2$ materials were then simulated and the resulting $\mathrm{P}$ vs. LLD curves were compared to the experimental data provided by CANMET ( $\mathrm{Xu}, 2010 \mathrm{a})$. While the simulations followed the same elastic curve, they were not able to achieve as high a peak loading value as the experimental work. Steady-state CTOAs from the DWTT simulations with the C2 materials ranged from $14^{\circ}-26^{\circ}$. These steady-state values are lower than the values measured from the SSY simulations, but that is to be expected as the SSY simulations were not able to propagate far enough to reach steady-state. These CTOAs are close, but slightly higher than the measured CTOAs reported by Xu et al (2010b). 
The TH materials were seen to be much less tough than the $\mathrm{C} 2$ materials, as it took the each of the TH materials less than $2.5 \mathrm{~mm}$ of applied displacement for $>25 \mathrm{~mm}$ of crack extension. It took $6.5 \mathrm{~mm}$ of applied displacement to make even the weakest $\mathrm{C} 2$ material (C2_1) propagate just $12.9 \mathrm{~mm}$. CTOA values for the $\mathrm{C} 2$ materials were also seen to be much higher than the $\mathrm{TH}$ materials.

While the two-dimensional simulation is useful for calibrating the cohesive parameters, a more sophisticated traction-separation law and a three-dimensional model will likely be needed to achieve a more complete picture of what occurs during the experimental drop-weight tear test. 


\begin{tabular}{|c|c|c|c|c|c|c|c|}
\hline $\operatorname{LLD}(\mathrm{mm})$ & $P(k N)$ & $\operatorname{LLD}(\mathrm{mm})$ & $P(k N)$ & $\operatorname{LLD}(\mathrm{mm})$ & $P(k N)$ & $\operatorname{LLD}(\mathrm{mm})$ & $P(k N)$ \\
\hline 0.0 & 10.5 & 4.0 & 213.6 & 16.0 & 163.7 & 28.0 & 65.0 \\
\hline 0.1 & 23.4 & 4.5 & 217.1 & 16.5 & 158.8 & 28.5 & 62.5 \\
\hline 0.2 & 41.2 & 5.0 & 220.2 & 17.0 & 153.7 & 29.0 & 60.0 \\
\hline 0.3 & 62.2 & 5.5 & 222.8 & 17.5 & 148.3 & 29.5 & 57.7 \\
\hline 0.4 & 82.3 & 6.0 & 222.7 & 18.0 & 144.0 & 30.0 & 55.6 \\
\hline 0.5 & 101.4 & 6.5 & 222.8 & 18.5 & 139.1 & 30.5 & 53.5 \\
\hline 0.6 & 119.4 & 7.0 & 221.1 & 19.0 & 134.7 & 31.0 & 51.0 \\
\hline 0.7 & 136.2 & 7.5 & 219.0 & 19.5 & 129.1 & 31.5 & 48.5 \\
\hline 0.8 & 150.0 & 8.0 & 217.7 & 20.0 & 123.9 & 32.0 & 46.7 \\
\hline 0.9 & 161.2 & 8.5 & 215.4 & 20.5 & 119.6 & 32.5 & 45.4 \\
\hline 1.0 & 169.3 & 9.0 & 212.1 & 21.0 & 115.7 & 33.0 & 44.0 \\
\hline 1.1 & 175.6 & 9.5 & 209.4 & 21.5 & 111.6 & 33.5 & 42.8 \\
\hline 1.2 & 180.2 & 10.0 & 206.1 & 22.0 & 107.8 & 34.0 & 41.4 \\
\hline 1.3 & 183.6 & 10.5 & 202.3 & 22.5 & 102.9 & 34.5 & 40.3 \\
\hline 1.4 & 186.1 & 11.0 & 198.9 & 23.0 & 98.5 & 35.0 & 39.3 \\
\hline 1.5 & 188.4 & 11.5 & 196.4 & 23.5 & 94.8 & 35.5 & 38.2 \\
\hline 1.6 & 190.3 & 12.0 & 192.3 & 24.0 & 90.7 & 36.0 & 37.2 \\
\hline 1.7 & 191.9 & 12.5 & 189.5 & 24.5 & 87.1 & 36.5 & 36.2 \\
\hline 1.8 & 193.5 & 13.0 & 186.6 & 25.0 & 83.6 & 37.0 & 34.5 \\
\hline 1.9 & 194.8 & 13.5 & 183.1 & 25.5 & 80.6 & 37.5 & 33.5 \\
\hline 2.0 & 196.0 & 14.0 & 180.6 & 26.0 & 77.1 & 38.0 & 32.6 \\
\hline 2.5 & 201.3 & 14.5 & 175.9 & 26.5 & 73.9 & 38.5 & 31.8 \\
\hline 3.0 & 205.8 & 15.0 & 171.2 & 27.0 & 70.8 & 39.0 & 31.0 \\
\hline 3.5 & 209.9 & 15.5 & 168.0 & 27.5 & 67.9 & 39.5 & 30.3 \\
\hline & & & & & & 40.0 & 29.5 \\
\hline
\end{tabular}

Table 5.1: P vs. LLD Data from CANMET - C2 Steel - Corrected for Compliance 


\begin{tabular}{|c|c|}
\hline LLD $(\mathrm{mm})$ & $\mathrm{P}(\mathrm{kN})$ \\
\hline 0.1 & 19.2 \\
0.2 & 39.9 \\
0.3 & 60.2 \\
0.4 & 79.0 \\
0.5 & 98.2 \\
0.6 & 115.9 \\
0.7 & 132.3 \\
0.8 & 146.3 \\
0.9 & 158.3 \\
1.0 & 168.3 \\
1.1 & 176.4 \\
1.2 & 182.7 \\
1.3 & 186.2 \\
1.4 & 188.1 \\
1.5 & 189.6 \\
1.6 & 190.9 \\
1.7 & 192.1 \\
1.8 & 193.2 \\
1.9 & 194.2 \\
2.0 & 195.2 \\
2.1 & 196.1 \\
2.2 & 196.9 \\
2.3 & 197.7 \\
2.4 & 198.5 \\
2.5 & 199.2 \\
2.6 & 199.9 \\
2.7 & 200.5 \\
2.8 & 201.1 \\
2.9 & 201.7 \\
3.0 & 202.3 \\
3.1 & 203.0 \\
3.2 & 203.6 \\
3.3 & 204.3 \\
3.4 & 204.9 \\
3.5 & 205.5 \\
3.6 & 206.0 \\
3.7 & 206.6 \\
3.8 & 207.1 \\
\hline
\end{tabular}

Table 5.2: P vs. LLD Sample data: Plane Strain Simulation 


\begin{tabular}{|c|c|c|c|c|c|c|c|}
\hline & & & & & & $\operatorname{LLD}(\mathrm{mm})$ & $P(k N)$ \\
\hline & & & & & & 0.00 & 0.00 \\
\hline & & & & & & 0.10 & 19.80 \\
\hline & & & & & & 0.20 & 41.41 \\
\hline & & & & & & 0.30 & 61.67 \\
\hline & & $\operatorname{LLD}(\mathrm{mm})$ & $P(k N)$ & & & 0.40 & 82.41 \\
\hline & & 0.00 & 0.00 & & & 0.50 & 101.29 \\
\hline & & 0.05 & 9.52 & & & 0.60 & 119.71 \\
\hline & & 0.10 & 19.80 & & & 0.70 & 137.28 \\
\hline & & 0.15 & 30.47 & & & 0.80 & 152.47 \\
\hline & & 0.20 & 41.38 & & & 0.90 & 164.99 \\
\hline & & 0.25 & 51.71 & & & 1.00 & 174.74 \\
\hline & & 0.30 & 61.92 & & & 1.05 & 178.63 \\
\hline & & 0.35 & 71.52 & $\operatorname{LLD}(\mathrm{mm})$ & $P(k N)$ & 1.10 & 181.72 \\
\hline & & 0.40 & 81.42 & 0.00 & 0.00 & 1.15 & 184.26 \\
\hline & & 0.45 & 91.04 & 0.10 & 19.80 & 1.20 & 186.41 \\
\hline & & 0.50 & 100.39 & 0.20 & 41.41 & 1.25 & 186.01 \\
\hline & & 0.55 & 109.63 & 0.30 & 61.66 & 1.30 & 186.15 \\
\hline & & 0.60 & 118.16 & 0.40 & 8239 & 1.35 & 186.13 \\
\hline & & 0.65 & 126.37 & 0.50 & 101.19 & 1.40 & 184.88 \\
\hline $\operatorname{LLD}(\mathrm{mm})$ & $\mathrm{P}(\mathrm{kN})$ & 0.70 & 133.86 & 0.60 & 119.54 & 1.45 & 183.37 \\
\hline 0.00 & 0.00 & 0.71 & 135.45 & 0.70 & 136.79 & 1.50 & 181.28 \\
\hline 0.05 & 9.52 & 0.72 & 136.80 & 0.80 & 150.65 & 1.55 & 169.98 \\
\hline 0.10 & 19.80 & 0.73 & 138.01 & 0.82 & 15343 & 1.60 & 171.13 \\
\hline 0.15 & 30.46 & 0.74 & 139.14 & 0.84 & 15528 & 1.65 & 167.06 \\
\hline 0.20 & 41.37 & 0.75 & 140.43 & 0.86 & 157.47 & 1.70 & 155.72 \\
\hline 0.25 & 51.69 & 0.76 & 141.64 & 0.88 & 158.87 & 1.75 & 149.86 \\
\hline 0.30 & 61.86 & 0.77 & 143.23 & 0.90 & 161.24 & 1.80 & 116.27 \\
\hline 0.31 & 63.40 & 0.78 & 143.16 & 0.92 & 162.55 & 1.85 & 111.94 \\
\hline 0.32 & 64.87 & 0.79 & 146.85 & 0.94 & 155.13 & 1.90 & 104.63 \\
\hline 0.33 & 67.12 & 0.80 & 139.65 & 0.96 & 167.73 & 1.95 & 77.00 \\
\hline 0.34 & 69.23 & 0.81 & 138.14 & 0.98 & 163.12 & 2.00 & 59.27 \\
\hline 0.35 & 71.30 & 0.82 & 135.22 & 1.00 & 162.55 & 2.05 & 34.62 \\
\hline 0.36 & 72.87 & 0.83 & 135.89 & 1.02 & 148.76 & 2.10 & 37.20 \\
\hline 0.37 & 52.19 & 0.84 & 133.98 & 1.04 & 144.15 & 2.15 & 32.02 \\
\hline 0.38 & 5.50 & 0.85 & 29.98 & 1.06 & 22.31 & 2.20 & 24.05 \\
\hline
\end{tabular}

Table 5.3 (a) - (d): Sample P vs. LLD data - TH Materials 


\begin{tabular}{|c|c|}
\hline$\Delta \mathrm{a}(\mathrm{mm})$ & LLD $(\mathrm{mm})$ \\
\hline 0.03 & 0.3206 \\
0.06 & 0.3356 \\
0.09 & 0.3465 \\
0.12 & 0.3555 \\
0.15 & 0.3595 \\
1.68 & 0.3615 \\
2.67 & 0.3619 \\
4.47 & 0.3623 \\
4.68 & 0.3627 \\
5.82 & 0.3631 \\
5.97 & 0.3635 \\
6.9 & 0.3639 \\
9.12 & 0.3643 \\
10.14 & 0.3647 \\
11.25 & 0.3651 \\
11.28 & 0.3667 \\
12.48 & 0.3698 \\
13.62 & 0.3706 \\
14.73 & 0.3710 \\
\hdashline 15.72 & 0.3714 \\
16.83 & 0.3718 \\
18.93 & 0.3722 \\
20.04 & 0.3726 \\
21.96 & 0.3730 \\
24.09 & 0.3734 \\
25.89 & 0.3738 \\
29.01 & 0.3746 \\
30 & 0.3750 \\
\hline
\end{tabular}

(a) $\mathrm{TH} 1$

\begin{tabular}{|c|c|}
\hline$\Delta \mathrm{a}(\mathrm{mm})$ & LLD $(\mathrm{mm})$ \\
\hline 0.03 & 0.3616 \\
0.15 & 0.4776 \\
0.21 & 0.5062 \\
0.27 & 0.5569 \\
0.36 & 0.5792 \\
0.42 & 0.5971 \\
0.45 & 0.6121 \\
0.48 & 0.6233 \\
0.51 & 0.6348 \\
0.54 & 0.6558 \\
0.84 & 0.6956 \\
0.87 & 0.7110 \\
1.02 & 0.7320 \\
1.11 & 0.7573 \\
2.01 & 0.7752 \\
2.88 & 0.7953 \\
3.81 & 0.8236 \\
10.08 & 0.8442 \\
15.48 & 0.8454 \\
21.18 & 0.8463 \\
25.11 & 0.8471 \\
\hline
\end{tabular}

(b) $\mathrm{TH} 2$

\begin{tabular}{|c|c|}
\hline$\Delta \mathrm{a}(\mathrm{mm})$ & LLD $(\mathrm{mm})$ \\
\hline 0.03 & 0.3676 \\
0.06 & 0.4163 \\
0.09 & 0.4487 \\
0.12 & 0.4730 \\
0.15 & 0.4974 \\
0.18 & 0.5130 \\
0.21 & 0.5280 \\
0.27 & 0.5856 \\
0.3 & 0.5984 \\
0.33 & 0.6198 \\
0.48 & 0.6541 \\
0.51 & 0.6583 \\
0.54 & 0.6883 \\
0.72 & 0.7397 \\
0.84 & 0.7867 \\
1.14 & 0.8509 \\
1.41 & 0.8765 \\
1.74 & 0.9021 \\
3.45 & 1.0048 \\
5.34 & 1.0519 \\
8.61 & 1.0818 \\
24.54 & 1.0904 \\
\hline
\end{tabular}

(c) $\mathrm{TH} 3$

\begin{tabular}{|c|c|}
\hline$\Delta a(\mathrm{~mm})$ & LLD $(\mathrm{mm})$ \\
\hline 0.03 & 0.3717 \\
0.15 & 0.5243 \\
0.21 & 0.5636 \\
0.3 & 0.6492 \\
0.48 & 0.7477 \\
0.6 & 0.8161 \\
0.81 & 0.8888 \\
0.9 & 0.9316 \\
1.02 & 0.9658 \\
1.17 & 1.0086 \\
1.2 & 1.0171 \\
1.26 & 1.0343 \\
1.38 & 1.0514 \\
1.53 & 1.1029 \\
1.8 & 1.1500 \\
2.1 & 1.2013 \\
2.37 & 1.2527 \\
2.88 & 1.3040 \\
3.3 & 1.3596 \\
3.6 & 1.3981 \\
4.14 & 1.4451 \\
4.71 & 1.5007 \\
6.87 & 1.5991 \\
8.01 & 1.7017 \\
11.04 & 1.8001 \\
15.84 & 1.9027 \\
20.13 & 2.0011 \\
25.2 & 2.0310 \\
\hline & \\
\end{tabular}

(d) $\mathrm{TH} 4$

Table 5.4 (a) - (d): Sample $\Delta$ a vs. LLD data - TH Materials 


\begin{tabular}{|c|c|c|c|c|c|c|c|}
\hline & & & & & & & \\
\hline & & & & $\Delta \mathrm{a}(\mathrm{mm})$ & $\operatorname{CTOA}\left({ }^{\circ}\right)$ & & \\
\hline & & & & 0.99 & 7.72 & & \\
\hline & & & & 1.02 & 7.66 & & \\
\hline & & & & 1.05 & 7.42 & & \\
\hline & & $\Delta \mathrm{a}(\mathrm{mm})$ & CTOA $\left({ }^{\circ}\right)$ & 1.08 & 7.30 & & \\
\hline & & 0.96 & 6.70 & 1.11 & 7.25 & & \\
\hline & & 0.99 & 6.56 & 1.14 & 7.36 & & \\
\hline & & 1.02 & 6.52 & 1.29 & 6.55 & & \\
\hline & & 1.05 & 6.19 & 1.32 & 6.46 & & \\
\hline$\Delta \mathrm{a}(\mathrm{mm})$ & $\operatorname{CTOA}\left({ }^{\circ}\right)$ & 1.08 & 6.15 & 1.38 & 6.19 & $\Delta \mathrm{a}(\mathrm{mm})$ & CTOA $\left({ }^{\circ}\right)$ \\
\hline 0.81 & 2.36 & 1.11 & 6.13 & 1.41 & 6.18 & 1.02 & 10.27 \\
\hline 1.68 & 2.08 & 1.14 & 6.22 & 1.44 & 6.32 & 1.08 & 9.83 \\
\hline 2.67 & 2.10 & 1.95 & 3.39 & 1.74 & 5.45 & 1.14 & 9.71 \\
\hline 4.47 & 2.02 & 1.98 & 3.68 & 1.77 & 5.53 & 1.32 & 8.99 \\
\hline 4.68 & 2.14 & 2.01 & 3.75 & 1.89 & 4.98 & 1.41 & 8.63 \\
\hline 5.82 & 1.78 & 2.64 & 3.36 & 1.92 & 4.98 & 1.50 & 8.79 \\
\hline 5.97 & 2.21 & 2.88 & 4.04 & 1.95 & 5.46 & $1.7 \underline{1}$ & 7.60 \\
\hline $6.90^{\circ}$ & 1.99 & 2.91 & 4.20 & 1.98 & 5.41 & 1.80 & 7.96 \\
\hline 9.12 & 1.91 & 3.78 & 4.30 & 2.01 & 5.43 & $2.0 \overline{1}$ & 7.07 \\
\hline 10.14 & 1.95 & 3.81 & 4.33 & 2.04 & 5.56 & 3.00 & 6.76 \\
\hline 11.25 & 2.02 & 4.77 & 3.07 & 3.18 & 3.59 & 4.14 & 5.88 \\
\hline 11.28 & 2.05 & 5.31 & 3.45 & 3.21 & 3.53 & 5.10 & 6.28 \\
\hline 12.48 & 1.99 & 5.67 & 3.98 & 3.24 & 3.72 & 6.81 & 4.50 \\
\hline 13.62 & 1.63 & 6.63 & 3.93 & 3.27 & 4.36 & 7.05 & 6.26 \\
\hline 14.73 & 1.81 & 6.81 & 3.12 & 3.45 & 5.09 & 8.37 & 5.99 \\
\hline 15.72 & 2.15 & 7.86 & 2.60 & 3.51 & 4.97 & 11.04 & 4.96 \\
\hline 16.83 & 1.87 & 7.89 & 2.95 & 3.54 & 5.02 & 12.90 & 4.20 \\
\hline 18.93 & 2.03 & 9.03 & 2.72 & 3.72 & 5.32 & 16.11 & 6.69 \\
\hline 20.04 & 1.96 & 10.08 & 3.01 & 3.84 & 5.31 & 19.20 & 4.62 \\
\hline 21.96 & 2.17 & 11.19 & 3.01 & 5.34 & 4.65 & 20.01 & 5.19 \\
\hline 24.09 & 1.90 & 13.35 & 3.02 & 6.54 & 3.65 & 25.20 & 4.81 \\
\hline 25.89 & 2.08 & 15.48 & 3.21 & 8.61 & 3.49 & 26.40 & 4.35 \\
\hline 26.88 & 2.02 & 17.7 & 3.41 & 16.14 & 4.61 & 28.71 & 4.25 \\
\hline 29.01 & 2.08 & 21.18 & 2.15 & 24.54 & 4.60 & 28.83 & 4.26 \\
\hline
\end{tabular}

Table 5.5 (a) - (d): Sample CTOA vs. $\Delta$ a data - TH Materials 


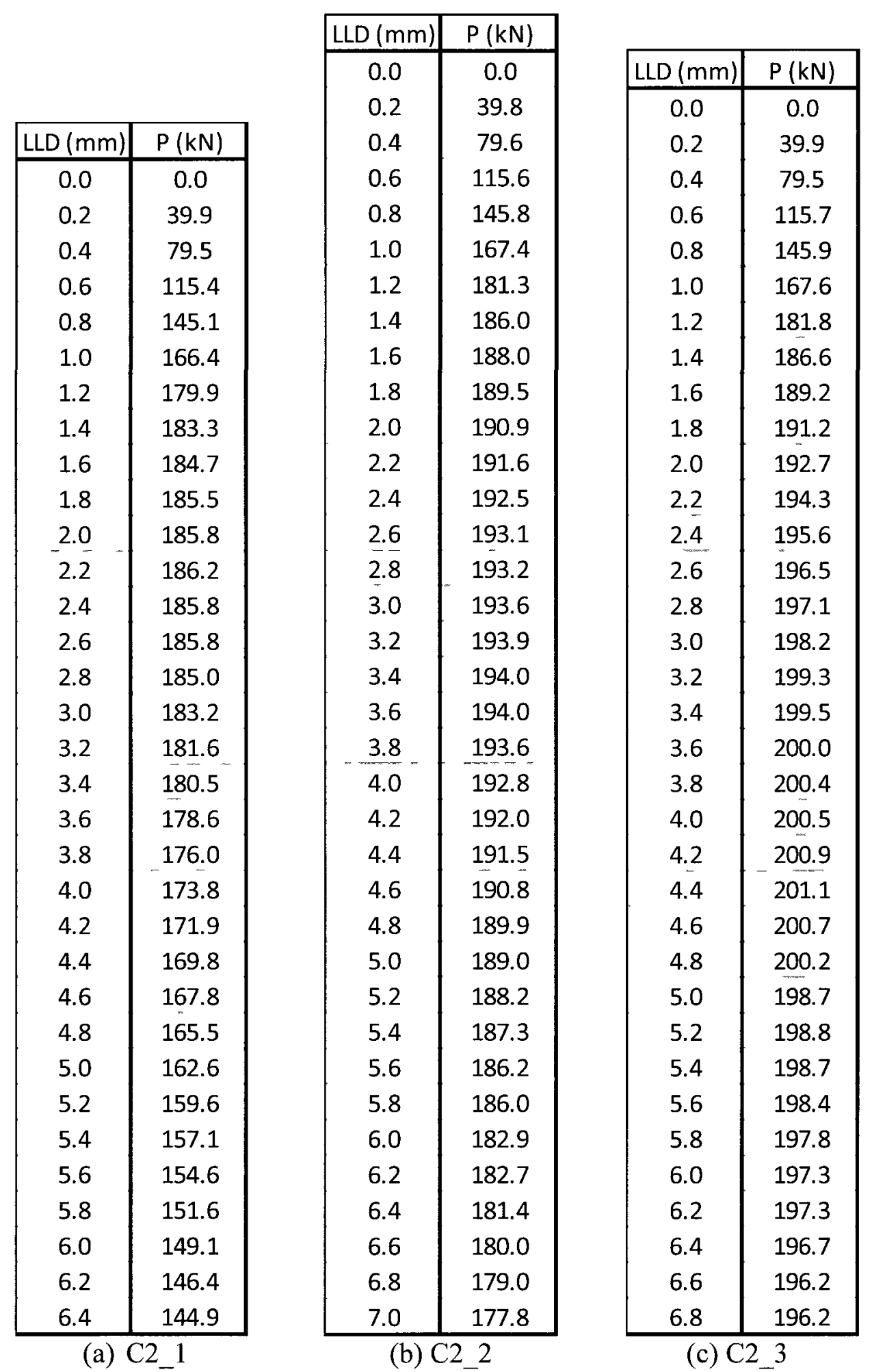

Table 5.6 (a) - (c): P vs. LLD data - C2 Materials 


\begin{tabular}{|c|c|}
\hline$\Delta \mathrm{a}(\mathrm{mm})$ & $\mathrm{LLD}(\mathrm{mm})$ \\
\hline 0.03 & 0.366 \\
0.21 & 0.769 \\
0.42 & 1.069 \\
0.60 & 1.278 \\
0.81 & 1.403 \\
1.02 & 1.585 \\
1.50 & 1.896 \\
2.01 & 2.189 \\
2.52 & 2.491 \\
3.00 & 2.744 \\
3.51 & 2.977 \\
4.02 & 3.201 \\
4.53 & 3.406 \\
5.01 & 3.593 \\
552 & 3.789 \\
6.00 & 3.934 \\
6.51 & 4.152 \\
7.02 & 4.372 \\
7.50 & 4.561 \\
8.01 & 4.748 \\
8.52 & 4.938 \\
9.00 & 5.099 \\
9.51 & 5.274 \\
10.02 & 5.460 \\
10.50 & 5.639 \\
11.01 & 5.816 \\
11.52 & 6.011 \\
12.00 & 6.150 \\
12.51 & 6.424 \\
12.87 & 6.564 \\
\hline
\end{tabular}

(a) $\mathrm{C} 2$

\begin{tabular}{|c|c|}
\hline$\Delta \mathrm{a}(\mathrm{mm})$ & $\mathrm{LLD}(\mathrm{mm})$ \\
\hline 0.03 & 0.544 \\
0.21 & 1.121 \\
0.42 & 1.447 \\
0.6 & 1.694 \\
0.81 & 1.899 \\
1.02 & 2.148 \\
1.5 & 2.626 \\
2.01 & 3.067 \\
2.52 & 3.571 \\
3 & 3.875 \\
3.51 & 4.249 \\
4.02 & 4.639 \\
4.5 & 5.004 \\
5.01 & 5.358 \\
5.52 & 5.816 \\
6 & 5.992 \\
6.51 & 6.339 \\
7.02 & 6.701 \\
7.5 & 7.052 \\
7.59 & 7.115 \\
\hline
\end{tabular}

(b) $\mathrm{C} 2 \_2$

\begin{tabular}{|c|c|}
\hline$\Delta \mathrm{a}(\mathrm{mm})$ & $\mathrm{LLD}(\mathrm{mm})$ \\
\hline 0.03 & 0.564 \\
0.21 & 1.297 \\
0.42 & 1.723 \\
0.6 & 2.017 \\
0.81 & 2.406 \\
1.02 & 2.648 \\
1.5 & 3.365 \\
2.01 & 3.998 \\
2.52 & 4.621 \\
3 & 4.921 \\
3.51 & 5.586 \\
4.02 & 6.158 \\
4.5 & 6.704 \\
4.65 & 6.880 \\
\hline
\end{tabular}

(c) $\mathrm{C} 2 \_3$

Table 5.7 (a) - (c): $\Delta$ a vs. LLD data - C2 Materials 


\begin{tabular}{|c|c|}
\hline$\Delta \mathrm{a}(\mathrm{mm})$ & CTOA $\left.^{\circ}\right)$ \\
\hline 0.96 & 26.45 \\
1.50 & 23.12 \\
2.01 & 19.60 \\
2.52 & 19.25 \\
3.00 & 18.57 \\
3.51 & 16.69 \\
4.02 & 16.09 \\
4.50 & 15.39 \\
5.01 & 14.48 \\
5.52 & 14.45 \\
6.00 & 13.43 \\
6.54 & 13.74 \\
7.02 & 15.90 \\
-7.50 & 14.95 \\
8.01 & 14.04 \\
8.52 & 14.19 \\
9.00 & 13.55 \\
9.51 & 12.97 \\
10.02 & 13.42 \\
10.50 & 13.78 \\
11.01 & 13.35 \\
11.52 & 13.61 \\
12.00 & 12.96 \\
12.51 & 14.27 \\
12.81 & 14.68 \\
\hline
\end{tabular}

(a) $\mathrm{C}_{2} 1$

\begin{tabular}{|c|c|}
\hline$\Delta \mathrm{a}(\mathrm{mm})$ & $\left.\mathrm{CTOA}^{\circ}\right)$ \\
\hline 1.02 & 39.10 \\
1.50 & 32.06 \\
2.01 & 28.09 \\
2.52 & 28.43 \\
3.00 & 25.91 \\
3.51 & 22.41 \\
4.02 & 24.78 \\
4.05 & 23.54 \\
4.08 & 23.39 \\
4.50 & 24.61 \\
5.01 & 23.47 \\
5.52 & 25.28 \\
6.00 & 21.59 \\
6.51 & 18.39 \\
7.02 & 23.63 \\
7.50 & 23.39 \\
7.59 & 22.12 \\
\hline
\end{tabular}

(b) $\mathrm{C} 2 \_2$

\begin{tabular}{|c|c|}
\hline$\Delta \mathrm{a}(\mathrm{mm})$ & CTOA $\left.^{\circ}\right)$ \\
\hline 1.02 & 50.66 \\
1.20 & 45.43 \\
1.41 & 42.33 \\
1.62 & 41.76 \\
1.80 & 38.41 \\
2.01 & 38.93 \\
2.22 & 38.07 \\
2.40 & 36.35 \\
2.61 & 35.32 \\
2.82 & 33.15 \\
3.00 & 28.83 \\
3.21 & 29.69 \\
3.42 & 30.67 \\
3.60 & 30.20 \\
3.81 & 30.91 \\
4.02 & 37.53 \\
4.20 & 34.14 \\
4.41 & 34.19 \\
4.62 & 34.78 \\
\hline
\end{tabular}

(c) $\mathrm{C} 2 \_3$

Table 5.8 (a) - (c): CTOA vs. $\Delta$ a data - C2 Materials 


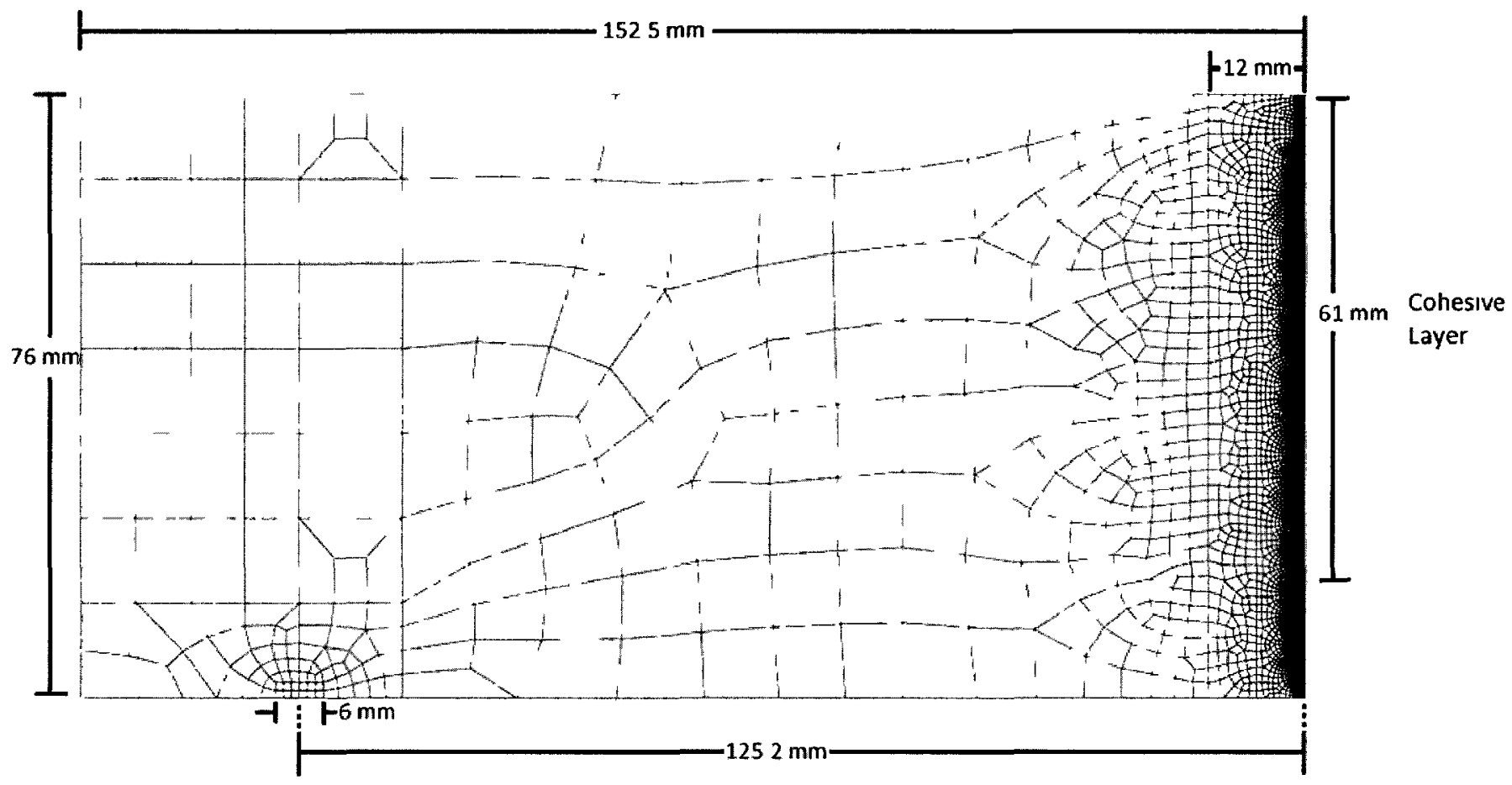

Figure 5.1: Model Geometry 


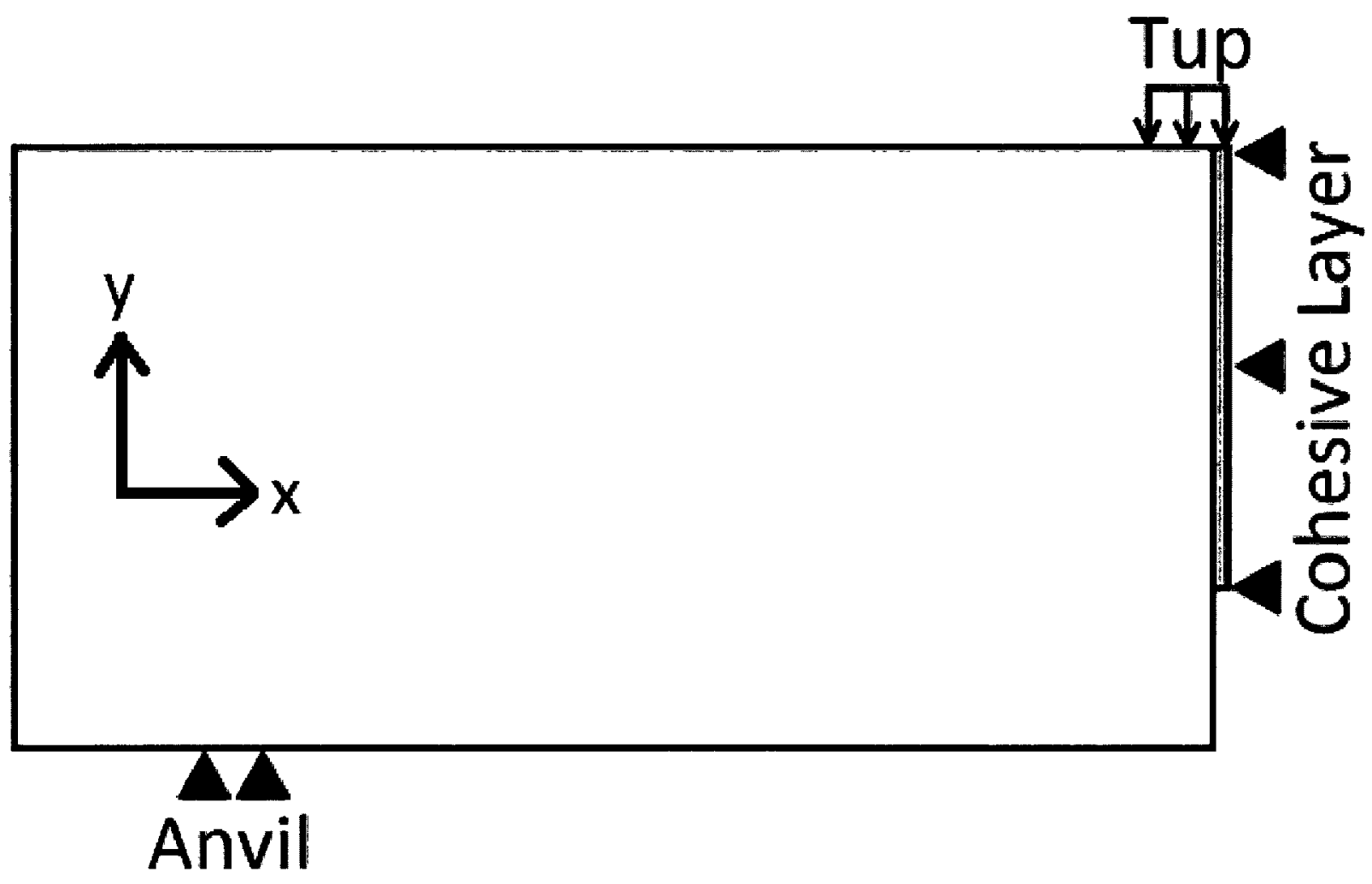

Figure 5.2: Loading Conditions

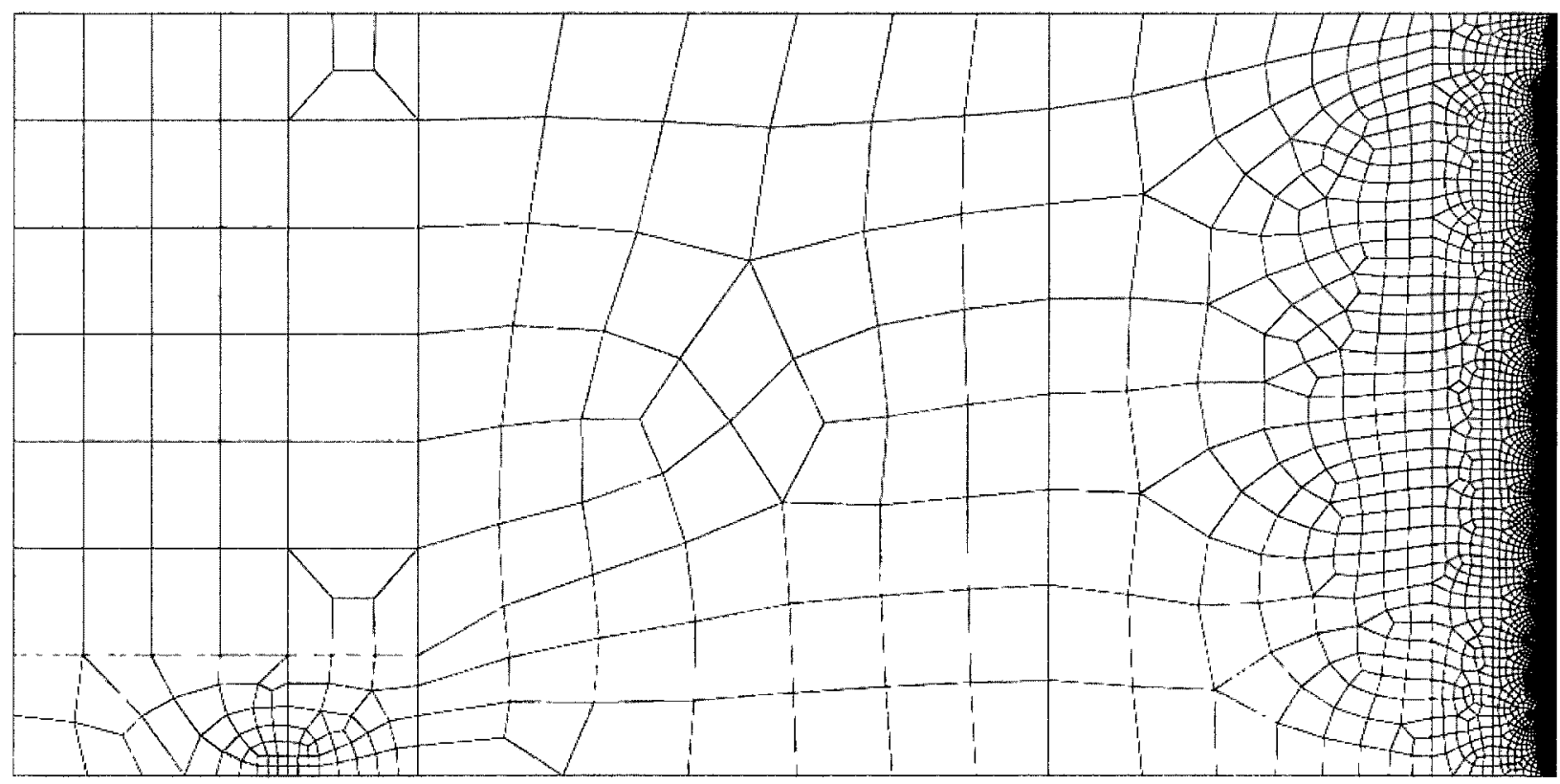

Figure 5.3: Mesh Design - Full Model 


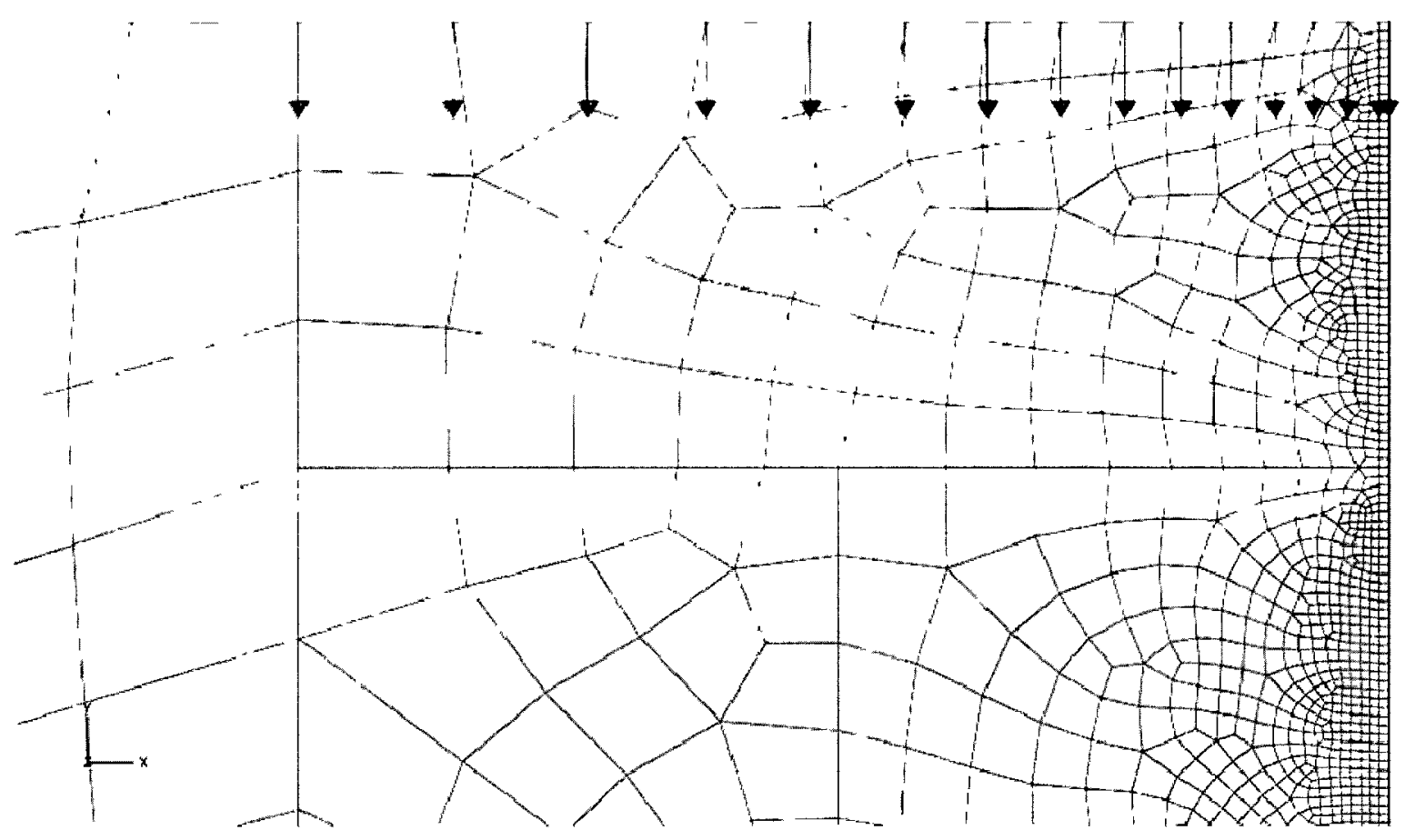

Figure 5.4: Mesh Design - Refinement at 'Tup'

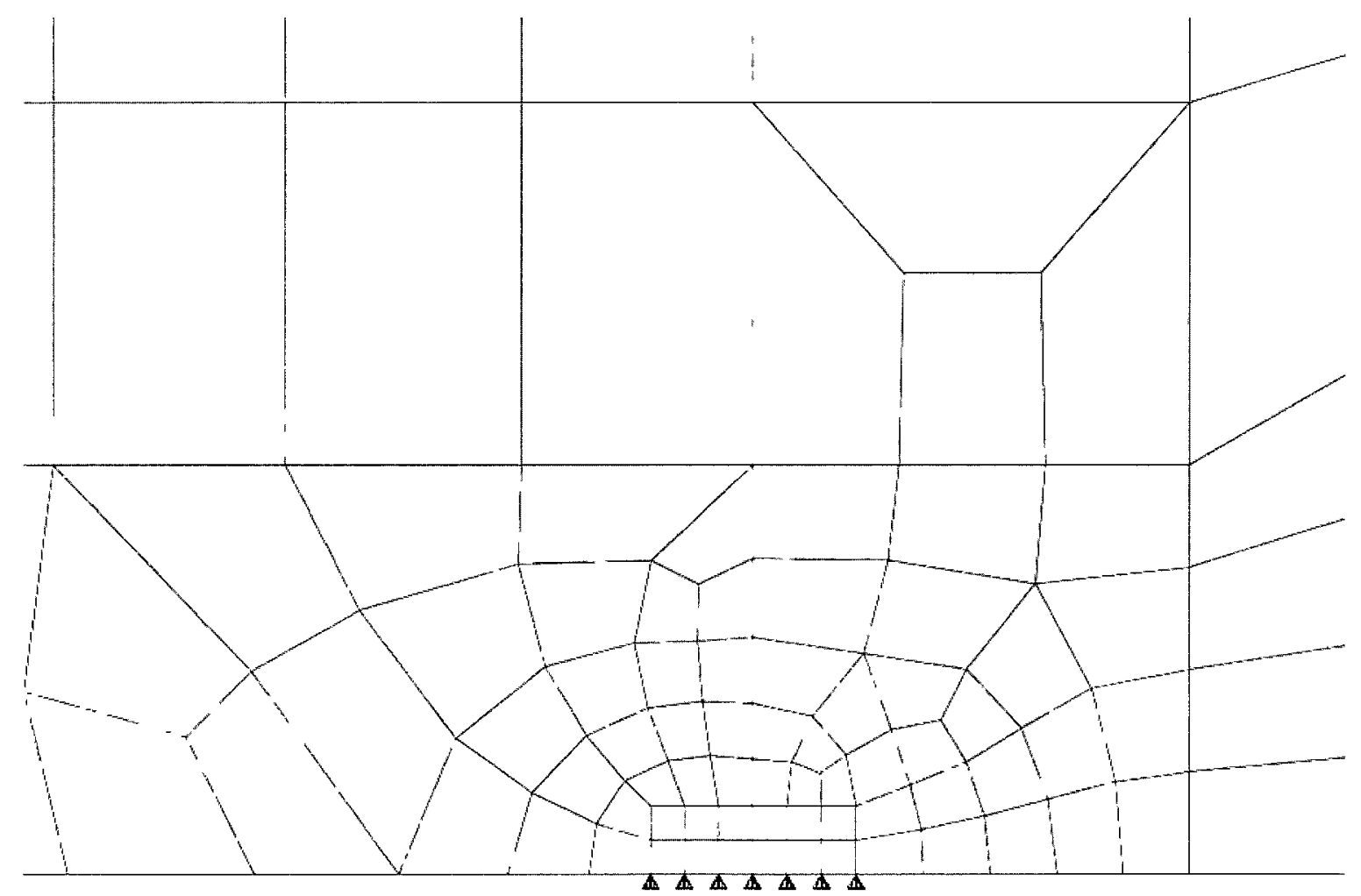

Figure 5.5: Mesh Design - Refinement at Anvil 


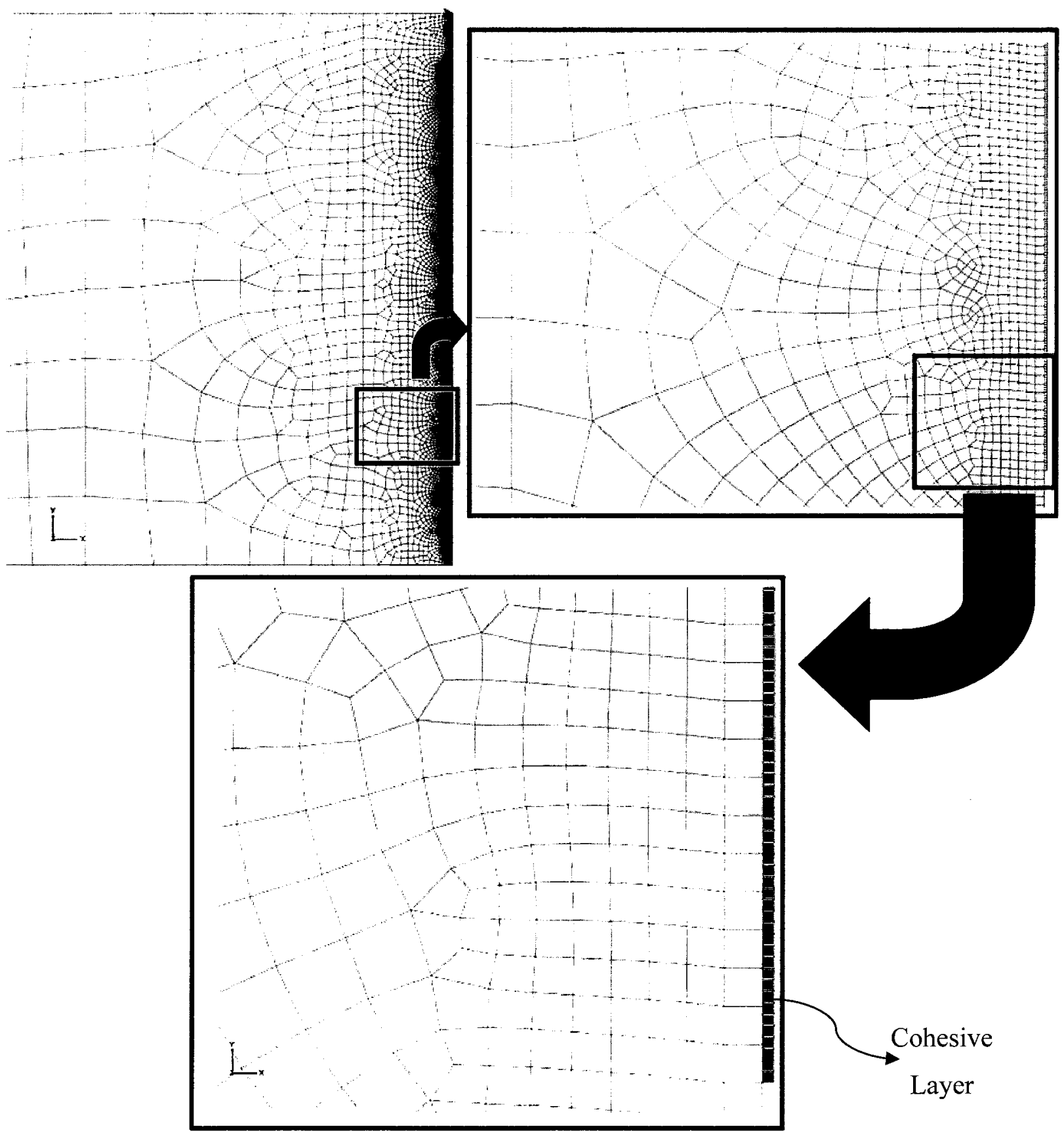

Figure 5.6: Mesh Design - Refinement at Crack Tip 


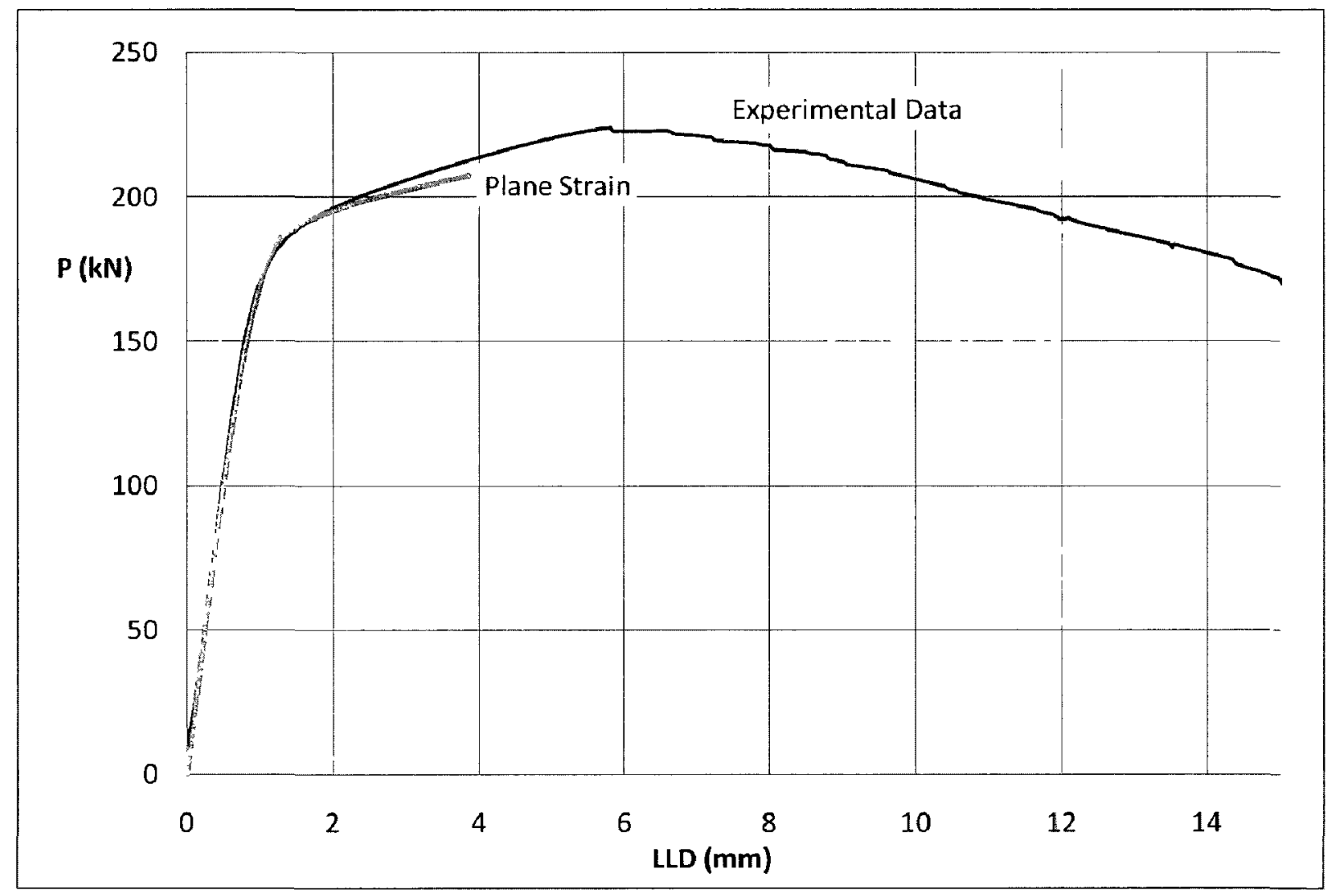

Figure 5.7: Model Verification - P vs. LLD curves -Plane Strain and Experimental Data 


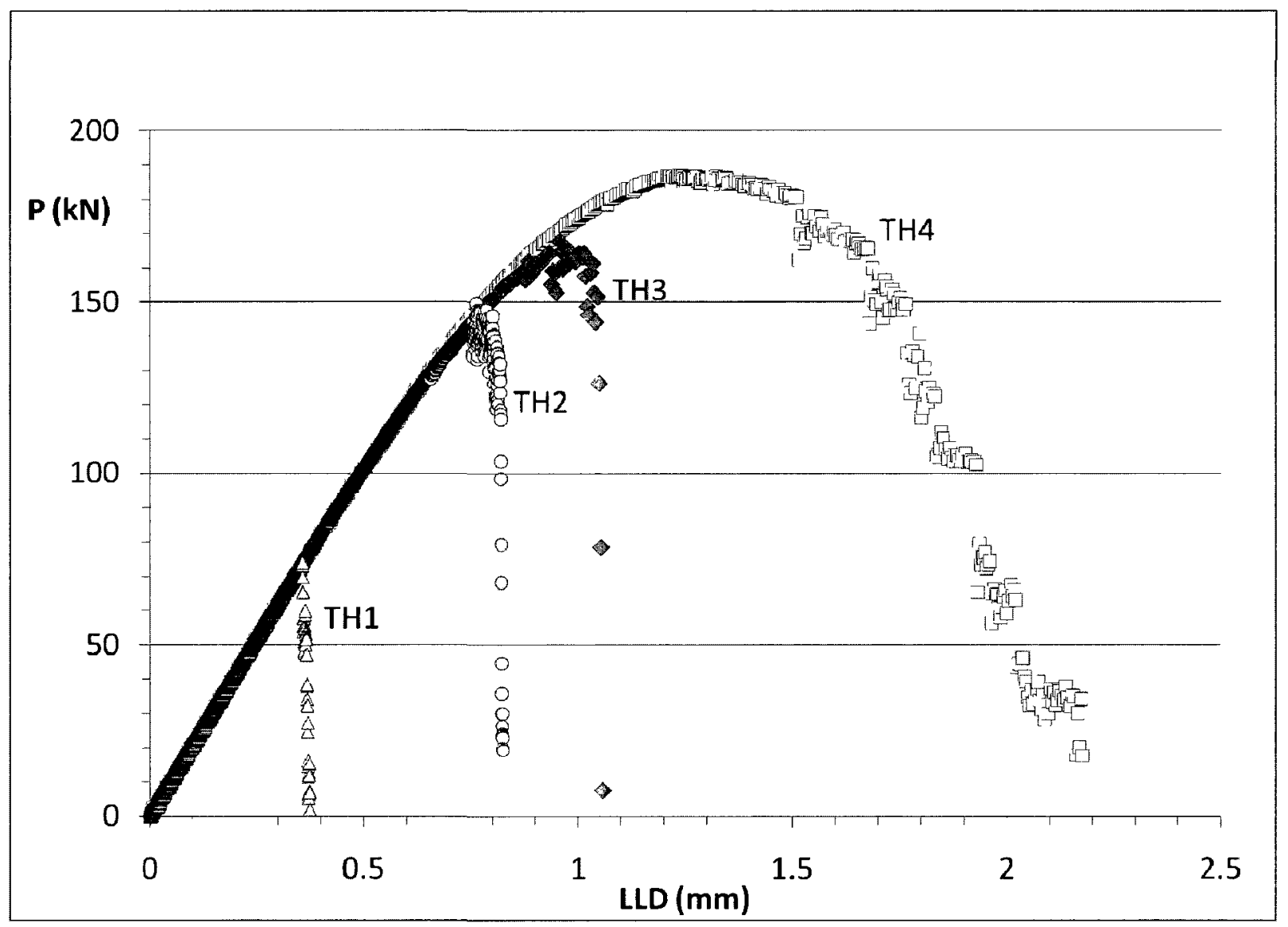

Figure 5.8: P vs. LLD - TH Materials 


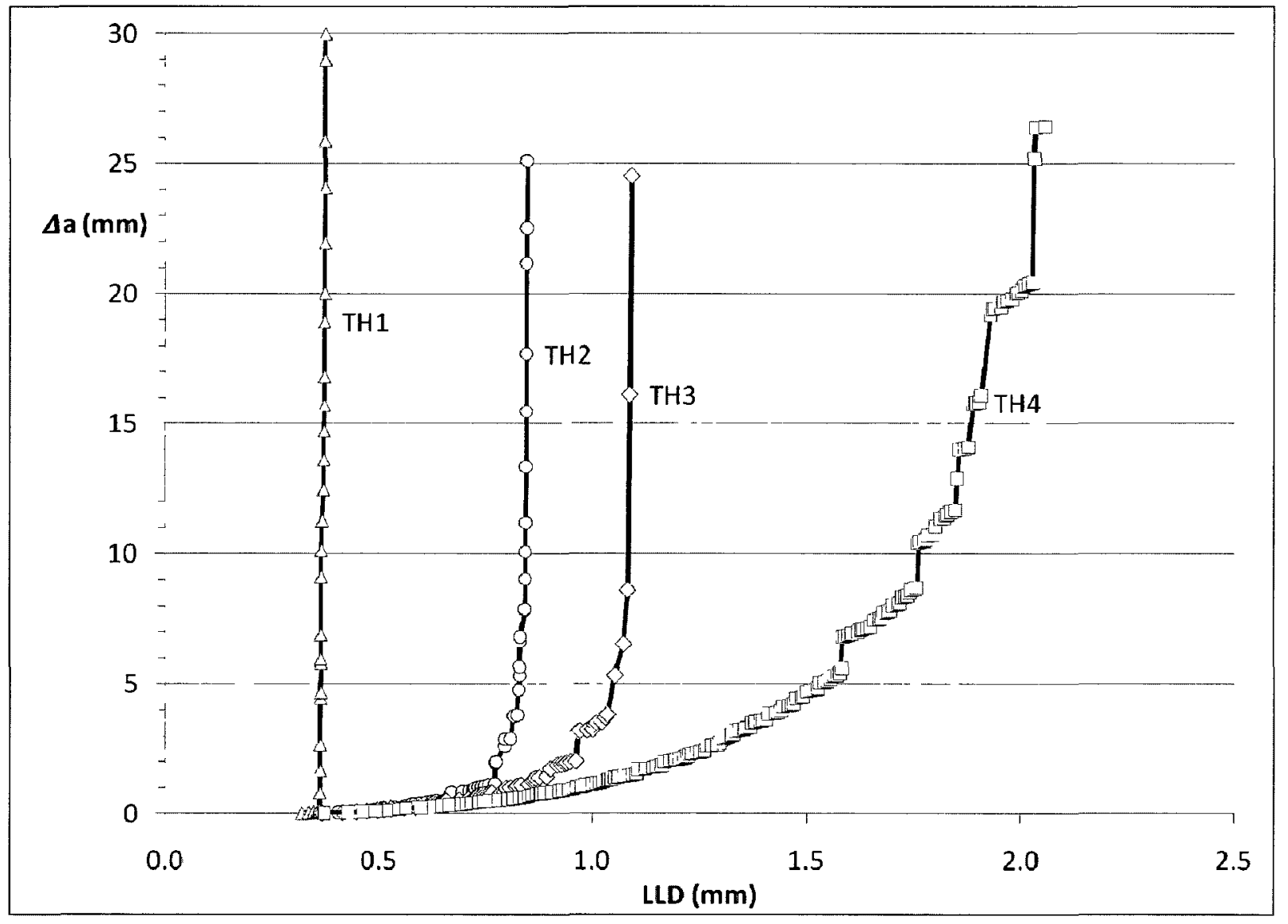

Figure 5.9: a vs. LLD - TH Materials 


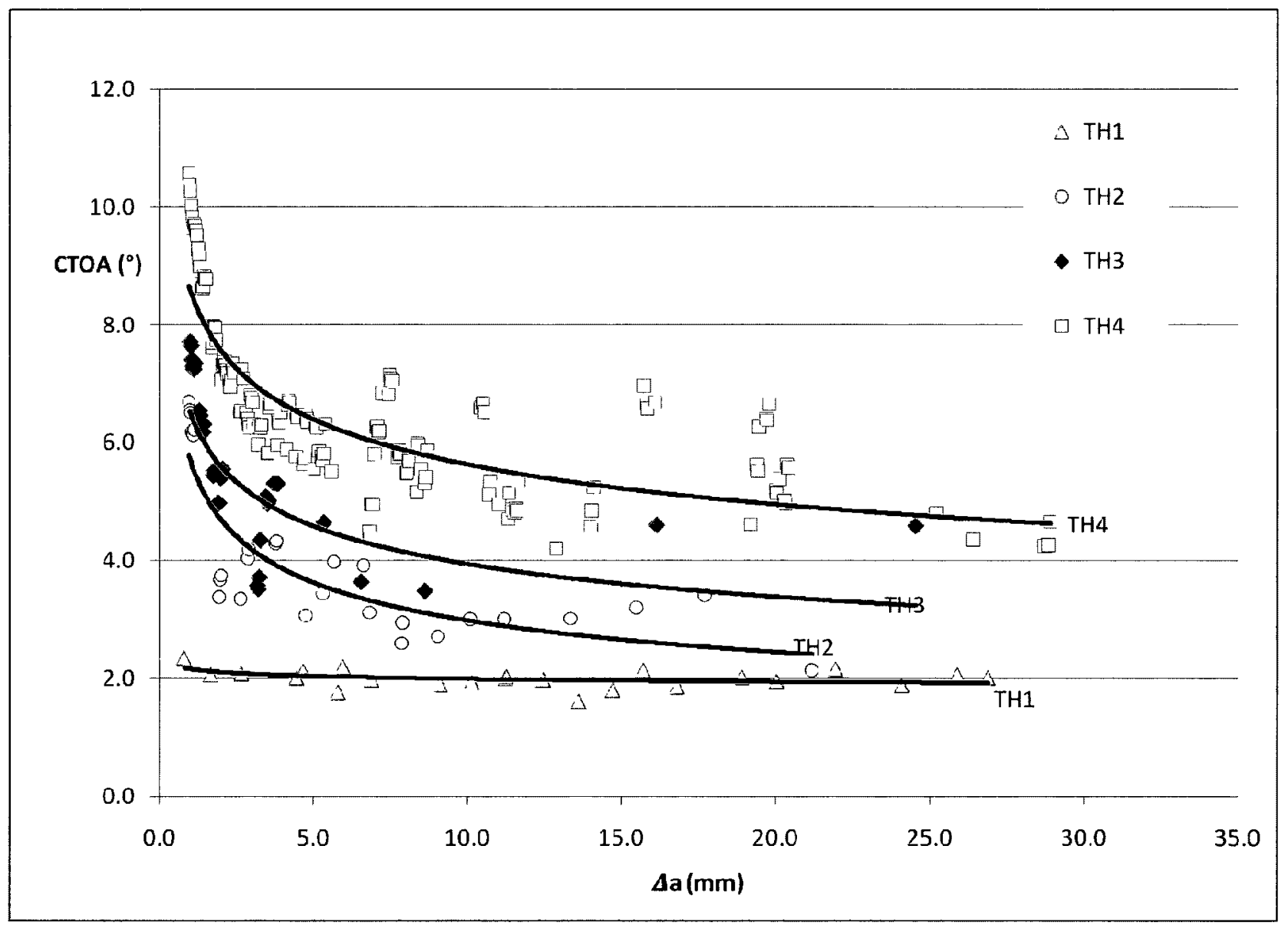

Figure 5.10: CTOA vs. a- TH Materials 


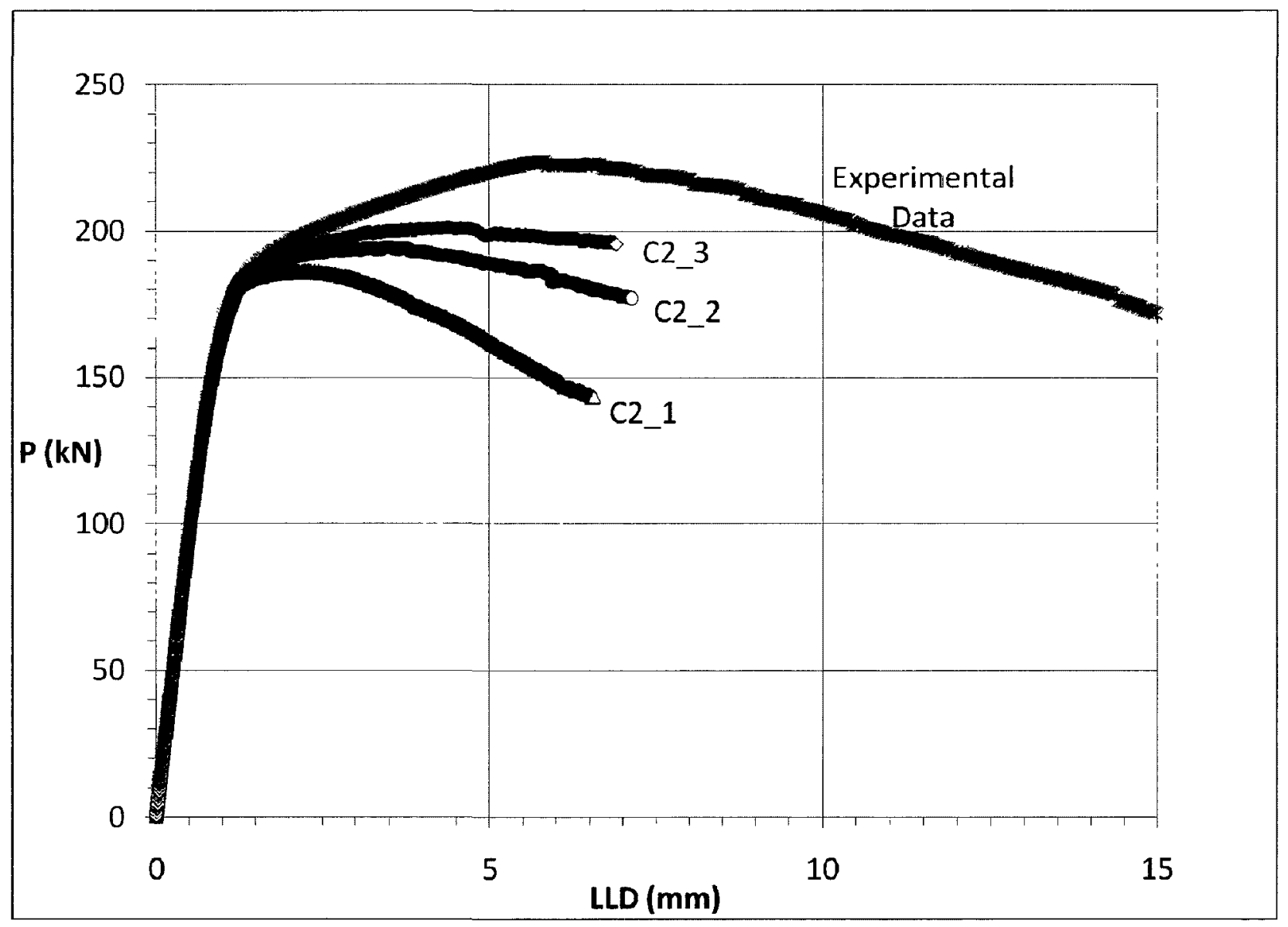

Figure 5.11: P vs. LLD - C2 Materials 


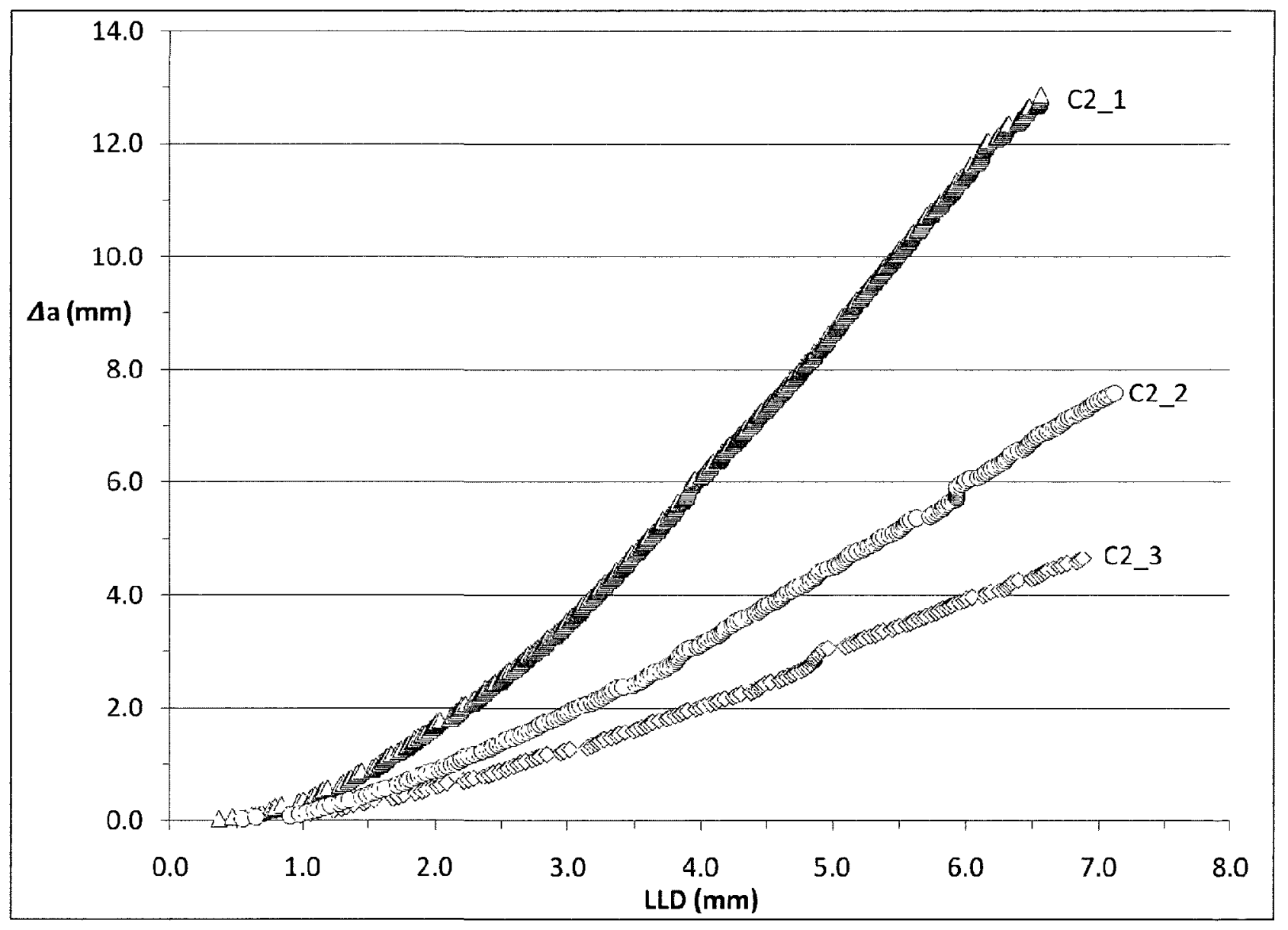

Figure 5.12: a vs. LLD - C2 Materials 


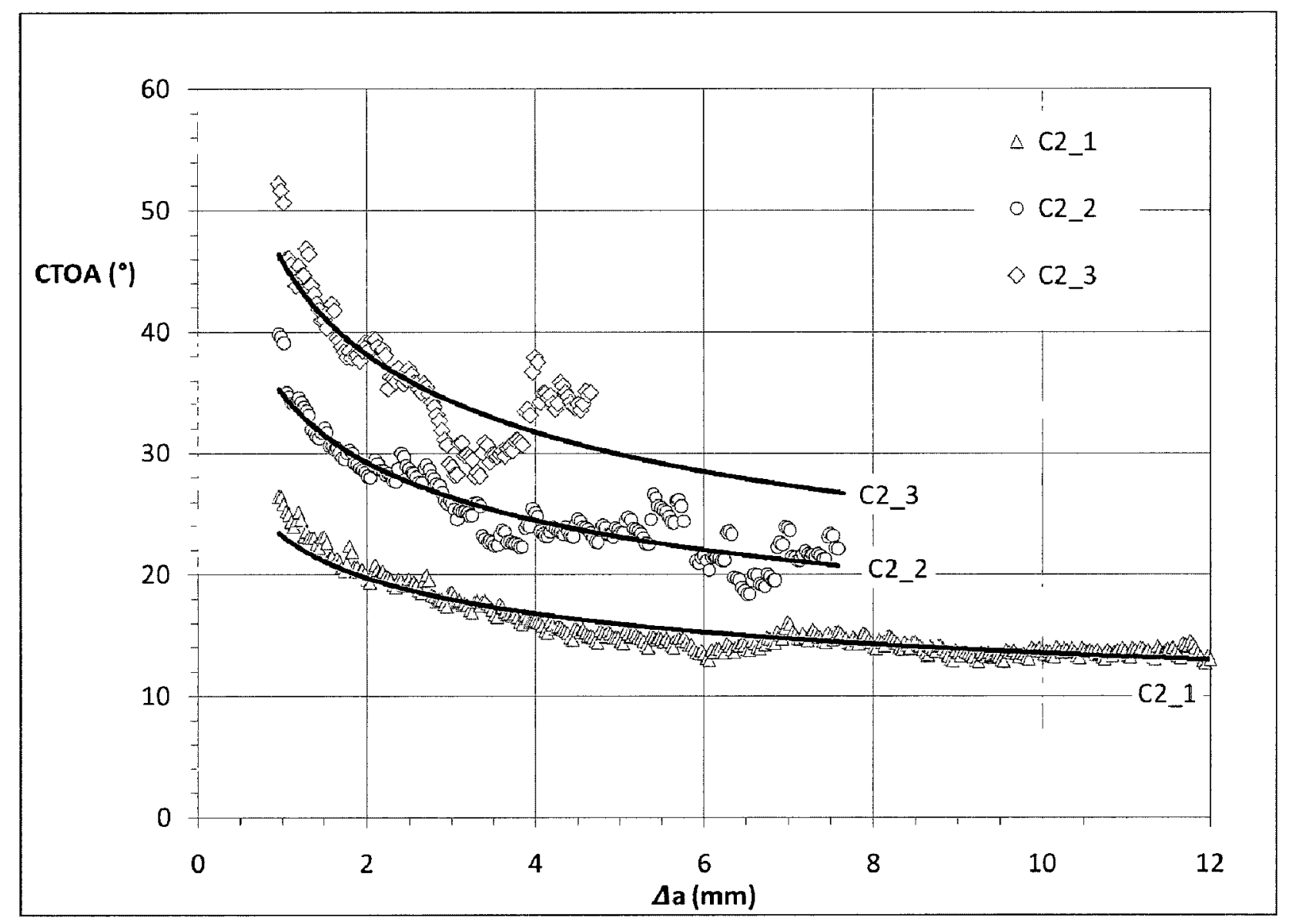

Figure 5.13: CTOA vs. a- C2 Materials 


\section{Chapter 6}

\section{Conclusions and Recommendations}

\subsection{Conclusions}

In this thesis, extensive finite element simulations of ductile crack propagation using cohesive zone modes were conducted. The following conclusions are summarized:

Unit cell models were used to verify the traction-separation laws that were implemented in this thesis. Two sets of materials were used in this thesis. The first set, the TH materials, were modeled after the ductile steel defined by Tvergaard and Hutchinson (1992). Four bilinear traction-separation laws, referred to as TH1-4, were used along with the shared elastic-plastic $\mathrm{TH}$ material. The second set of materials is referred to as the C2 materials. They were modeled after an X70 steel characterised by Xu (2010a). Three bilinear traction-separation laws were associated with the $\mathrm{C} 2$ elastic-plastic material. To verify the TS laws, a unit cell analysis was performed in Abaqus/Explicit (2008) for each of the seven laws used. All seven materials were found to respond exactly as expected.

Small-scale yielding simulations were performed to model crack propagation using the seven TH and C2 materials. An elastic-plastic simulation, with no cohesive elements, was carried out using the TH material, and it was verified that the stress intensity at the crack tip closely matched the expected stress intensity due to the applied displacement field. From the simulations with cohesive zone elements, the resistance curves $\left(K_{r} / K_{0}\right.$ vs. $\left.\Delta a / R_{0}\right)$ determined from simulation for the four TH materials were found to agree quite closely with those published by Tvergaard and Hutchinson (1992). The resistance curves for the three C2 materials were also determined. Crack tip opening angle data was measured for all seven simulations. Typical values of steady-state CTOA ranged from $2^{\circ}-6^{\circ}$ for $\mathrm{TH} 1-4$ respectively. While steady-state values of CTOA were not 
achieved for the $\mathrm{C} 2$ materials due to the short length of the cohesive zone, the CTOA at initiation ranged from $30^{\circ}-80^{\circ}$. The CTOAs for the $\mathrm{C} 2$ materials ranged from $20^{\circ}-60^{\circ}$ after $2 \mathrm{~mm}$ of propagation.

Drop-weight tear test (DWTT) simulations were carried out for the seven TH and C2 materials. A plane strain elastic-plastic analysis was carried out for the $\mathrm{C} 2$ material with no cohesive elements, and the resulting load vs. load-line displacement data was found to agree quite closely to the experimental observations $(\mathrm{Xu}, 2010 \mathrm{a})$ before crack initiation. Simulations were then carried out with cohesive zone elements. Load vs. load-line displacement data was collected for all seven simulations, and the $\mathrm{C} 2$ simulations were seen to have reasonable agreement with the experimental data (Xu, 2010a). Crack length vs. load-line displacement data was collected for all seven simulations. CTOA data was also collected for all simulations. The steady-state CTOAs of the TH materials during the DWTT simulations were in the range of $2^{\circ}-4.5^{\circ}$, which is in close agreement to the steady-state CTOAs measured from the SSY simulations. The estimated steadystate CTOAs of the C2 materials during the DWTT simulations were in the range of $14^{\circ}-26^{\circ}$. While these values are lower than those measured from the SSY results, that is to be expected as the SSY simulations did not have time to reach steady-state. These values are also reasonably close to the optically measured CTOA of $18.5^{\circ}$, and the CTOA calculated by the simplified single-specimen method of $12.4^{\circ}$ (Xu et al, 2010b).

Overall, cohesive zone (CZ) modeling can be used to simulate crack propagation in ductile steels, as has been demonstrated for the two steels referred to here as $\mathrm{TH}$ and $\mathrm{C} 2$. It is possible to consistently measure the CTOA when using CZ modeling. Various TS laws have been investigated, and the effects of toughening the cohesive parameters were found to be consistent with expectations. Higher peak tractions and fracture energies led to increased fracture resistance, and increased values for steady-state CTOA, as demonstrated by both the TH and $\mathrm{C} 2$ simulations. The measured CTOAs agreed reasonably between the SSY and DWTT simulations for both the $\mathrm{TH}$ and $\mathrm{C} 2$ material sets. It is therefore likely that these CTOA values would be transferable to real structures such as pipelines. Direct comparison between the C2 material's 
CTOAs measured from the DWTT simulations and the experimental observations ( $\mathrm{Su}, 2010 \mathrm{~b}$ ), had reasonable agreement.

\subsection{Recommendations for Future Work}

The long-term goal of the research in this area is to develop stable and computationally-efficient cohesive zone models that could be used to predict fracture of full-scale pipeline specimens. A secondary goal is to improve the measurement of CTOA by gaining a more thorough understanding of the crack tip. Many steps must be completed to get this work to that point.

The first step is to gain a more thorough understanding of the results found during this work. More work needs to be done to understand the differences between the published data by Tvergaard and Hutchinson (1992), and the data found in this work for the TH materials (Figure 4.5). It is likely that the shape of the TS law played a large role in the discrepancies. These simulations should be repeated using alternative TS law shapes, such as trapezoidal, exponential and linear-parabolic, to analyze the effect that shape has on fracture resistance of both the SSY and DWTT models, as well as the effect that shape has on the CTOA measurements from these two models. Additionally, more work should be done to understand the oscillations seen in the CTOA data presented in Figures 4.9, 5.10, and 5.13. It remains unknown whether this is caused by the stiffness of the cohesive elements, or a dynamic effect of the explicit simulations. Finally, work should be done to see if the C2 material's DWTT simulations could be fine-tuned to allow for additional crack propagation. The abortion of these simulations due to numerical difficulties prevents the CTOA from reaching its steady-state values.

A second step would be to improve upon the two-dimensional results found here. For example, the current SSY model did not have a long enough cohesive zone to reach steady-state fracture propagation for all of the materials used. Extending this $\mathrm{CZ}$ could increase the confidence in the quoted steady-state values. Secondly, parametric studies could be performed to isolate the effect that each of the three bilinear TS law parameters has on fracture resistance and CTOA 
measurements. These parametric studies could lead to closer representation of the experimental observations by the numerical simulations. Moreover, these simulations could be repeated using plane stress conditions, rather than plane strain, to determine the effect on fracture resistance and CTOA measurements. The real test specimen, with a thickness of around $13 \mathrm{~mm}$, is actually somewhere between the two conditions.

Finally, the TS laws used here should be applied to three-dimensional SSY and DWTT models to investigate their ability to predict trends such as crack tunnelling. Furthermore, while it seems that $3 \mathrm{D}$ effects such as slant-fracture would be impossible to model using the current state of the cohesive zone elements in Abaqus, it could be possible to model this using Extended Finite Element Methods (XFEM) techniques. 


\section{References}

Abaqus (2008), Abaqus Analysis User's Manual. Abaqus Online Documentation, Dassault Systèmes, Version 6.8 .

ASTM E1820-01 (2001), Standard Test Method for Measurement of Fracture Toughness, ASTM International

ASTM E2472-06 (2006), Standard Test Method for Determination of Resistance to Stable Crack Extension under Low-Constraint Conditions, ASTM International

G. Alfano (2006), On the influence of the shape of the interface law on the application of cohesive-zone models, Composites Science and Technology, Vol. 66, pp. 723-730

G.I. Barenblatt (1962), The mathematical theory of equilibrium cracks in brittle fracture, Advances in Applied Mechanics, Vol. 7, pp. 55-129

R. de Borst (2003), Numerical aspects of cohesive-zone models. Engineering Fracture Mechanics, Vol. 70, pp. 1743-1757

N. Chandra, H. Li, C. Shet, H. Ghonem (2002), Some issues in the application of cohesive zone models for metal-ceramic interfaces. International Journal of Solids and Structures, Vol. 39, pp. 2827-2855

C.R. Chen, O. Kolednik, I. Scheider, T. Siegmund, A. Tatschl, F.D. Fischer (2003), On the determination of the cohesive zone parameters for the modeling of micro0ductile crack growth in thick specimens. International Journal of Fracture, Vol. 120, pp. 517-536

A. Cornec, I. Scheider, K-H. Schwalbe (2003), On the practical application of the cohesive model, Engineering Fracture Mechanics, Vol. 70, pp. 1963-1987

T. Diehl (2008), On using a penalty-based cohesive-zone finite element approach, Part I: Elastic solution benchmarks, International Journal of Adhesion and Adhesives, Vol. 28, pp. 237-255

D.S. Dugdale (1960), Yielding of steel sheets containing slits, Journal of the Mechanics and Physics of Solids, Vol. 8, pp. 100-104 
M. Elices, G.V. Guinea, J. Gómez, J. Planas (2002). The cohesive zone model: advantages, limitations and challenges. Engineering Fracture Mechanics, Vol. 69, pp. 137-163

D.J. Horsley (2003), Background to the use of CTOA for prediction of dynamic ductile fracture arrest in pipelines. Engineering Fracture Mechanics, Vol. 70, pp. 547-552.

ISO 22889:2007(E) (2007), Metallic materials - Method of test for the determination of resistance to stable crack extension using specimens of low constraint. International Standards Organization

K. Keller, S. Weihe, T. Siegmund, B. Kröplin (1999), Generalized cohesive zone model: incorporating triaxiality dependent failure mechanisms. Computational Materials Science, Vol. 16, pp. 267-274.

A. Martinelli, S. Venzi (1996), Tearing modulus, J-integral, CTOA and crack profile shape obtained from the load-displacement curve only. Engineering Fracture Mechanics, Vol. 53, pp. 263-277.

W. Maxey, J.F. Keifner, R.J. Eiber (1976), Ductile Fracture Arrest in Gas Pipelines, A.G.A. catalogue number L32176

J.C. Newman, M.A. James, U. Zerbst (2003) A review of the CTOA/CTOD fracture criterion. Engineering Fracture Mechanics, Vol. 70, pp. 371-385

J. R. Rice, E. P. Sorensen (1978), Continuing crack-tip deformation and fracture for plane-strain crack growth in elastic-plastic solids. Journal of the Mechanics and Physics of Solids, Vol. 26, pp. $163-186$

I. Scheider, W. Brocks (2006), Cohesive elements for thin-walled structures. Computational Materials Science, Vol. 37, pp. 101-109

D.J. Shim, G. Wilkowski, D.M. Duan, J. Zhou (2010a), Effect of fracture speed on ductile fracture resistance - Part 1: Experimental. Proceedings of the 8th International Pipeline Conference, IPC2010-31310. 
D.J. Shim, G. Wilkowski, D.M. Duan, J. Zhou (2010b), Effect of fracture speed on ductile fracture resistance - Part 2: Results and application. Proceedings of the 8th International Pipeline Conference, IPC2010-31021.

V. Tvergaard, J.W. Hutchinson, (1992), The relation between crack growth resistance and fracture process parameters in elastic-plastic solids. Journal of the Mechanics and Physics of Solids, Vol. 40, pp. 1377-1397

Williams, M.L., (1957), On the stress distribution at the base of a stationary crack. J. Appl. Mech Vol. 24, pp. 109-114

S. Xu, R. Bouchard, W.R. Tyson (2004), Flow Behavior and Ductile Fracture Toughness of a High Toughness Steel. Proceedings of IPC 2004, IPC04-192

S. Xu, R. Bouchard, W.R. Tyson (2007). Simplified single-specimen method for evaluating CTOA. Engineering Fracture Mechanics, Vol. 74, pp. 2459-2464.

S. Xu, W.R. Tyson (2008), CTOA Measurement of Pipe Steels Using DWTT Specimen. Proceedings of IPC 2008, IPC2008-64060

S. Xu, W.R. Tyson, R. Bouchard (2009). "Experimental Validation of Simplified SingleSpecimen CTOA Method for DWTT Specimens", Proceedings of $12^{\text {th }}$ International Conference on Fracture (ICF 12), July 12-17, Ottawa, ON, Canada, ICF2009-269

S. Xu (2010a), Private correspondence between S. Xu and Andrew Dunbar regarding experimental results of a DWTT for a C2 (X70) Steel and it's stress-strain curve.

S. Xu, W.R. Tyson, R. Eagleson, C.N. McCowan, E.S. Drexler, J.D. McColskey, Ph.P. Darcis (2010b), Measurement of CTOA of pipe steels using MDCB and DWTT specimens, Proceedings of the 8th International Pipeline Conference, IPC2010-31076.

S. Xu, G. Shen, G. Roy, W.R. Tyson (2011a), Application of plastic hinge model to single-edgenotched bend SE(B) specimen, Report No. 2011-01(TR), CANMET-MTL, Ottawa, ON, Canada, April 2011 
S. Xu, W.R. Tyson (2011b), Recommended practice for determination of crack-tip opening angle of structural steels using DWTT specimens, Report No. 2011-03(TRR), CANMET-MTL, Ottawa, ON, Canada, May 2011 